\title{
INVESTIGATING THE EFFECTS OF SOCIAL AND TEMPORAL DYNAMICS IN FITNESS GAMES ON CHILDREN'S PHYSICAL ACTIVITY
}

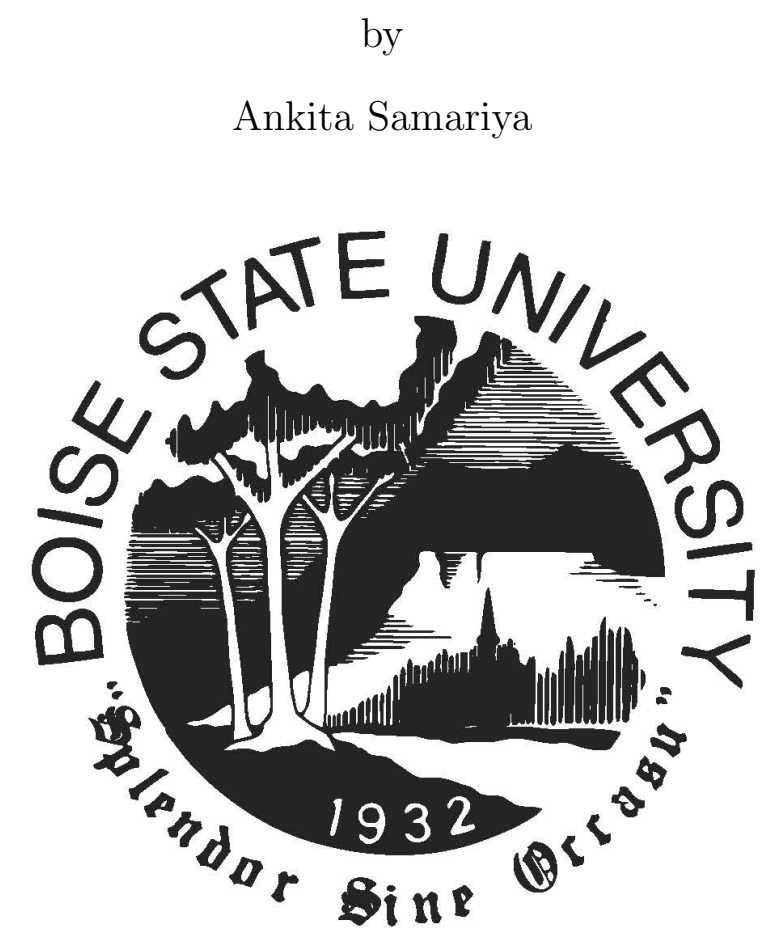

\author{
A thesis \\ submitted in partial fulfillment \\ of the requirements for the degree of \\ Master of Science in Computer Science \\ Boise State University
}

August 2019 
(c) 2019

Ankita Samariya

ALL RIGHTS RESERVED 
BOISE STATE UNIVERSITY GRADUATE COLLEGE

DEFENSE COMMITTEE AND FINAL READING APPROVALS

of the thesis submitted by

Ankita Samariya

Thesis Title: Investigating the Effects of Social and Temporal Dynamics in Fitness Games on Children's Physical Activity

Date of Final Oral Examination: 3rd May 2019

The following individuals read and discussed the thesis submitted by student Ankita Samariya, and they evaluated the presentation and response to questions during the final oral examination. They found that the student passed the final oral examination.

Jerry Alan Fails, Ph.D. Chair, Supervisory Committee

Francesca Spezzano, Ph.D. Member, Supervisory Committee

Maria Soledad Pera, Ph.D. Member, Supervisory Committee

The final reading approval of the thesis was granted by Jerry Alan Fails, Ph.D., Chair of the Supervisory Committee. The thesis was approved by the Graduate College. 


\section{ACKNOWLEDGMENTS}

This work would not have been possible without the thoughtful support of many who took a personal interest in its completion. The author would like to thank her

husband, who has supported her every step of the way. The author would also like to thank God, for the good mental and physical health required for finishing this thesis. The author is also very grateful for the immense time and effort contributed by Dr. Jerry Fails. His guidance and insight were a driving force in completing this work. The author would also like to thank Boise State University and the faculty of the Computer Science department for awarding an assistantship, without which this work would not have been possible. 


\begin{abstract}
Obesity in children has more than doubled in the last four decades. A variety of fitness technologies such as activity trackers, exergames, and mobile applications are available to promote physical activity, but few are designed adequately to meet children's specific needs. In my initial research, I found that children prefer having social interaction, a narrative, and flexibility while interacting with such technologies. Other research has shown that persuasive displays encouraged adults towards physical activity. As a part of this work, a collection of mobile applications, collectively known as KidFit Suite were developed. These applications encourage children to be physically active leveraging social and temporal aspects. I present findings from a user study conducted on 16 children (ages 6-11) who utilized the mobile applications in KidFit Suite. The applications are primarily evaluated on the spectrums of social engagement and temporal flexibility. In the study, children engaged with the mobile application and liked both the collaborative and competitive aspects of social interaction. In terms of temporal flexibility, they preferred short synchronous games better than longer asynchronous games.
\end{abstract}




\section{TABLE OF CONTENTS}

ABSTRACT $\ldots \ldots \ldots \ldots \ldots \ldots \ldots \ldots \ldots \ldots \ldots \ldots \ldots \ldots \ldots \ldots$

LIST OF TABLES $\ldots \ldots \ldots \ldots \ldots \ldots \ldots \ldots \ldots \ldots \ldots \ldots \ldots \ldots \ldots \ldots \ldots$

LIST OF FIGURES $\ldots \ldots \ldots \ldots \ldots \ldots \ldots \ldots \ldots \ldots \ldots \ldots \ldots \ldots \ldots \ldots$

LIST OF ABBREVIATIONS $\ldots \ldots \ldots \ldots \ldots \ldots \ldots \ldots \ldots \ldots$ xiv

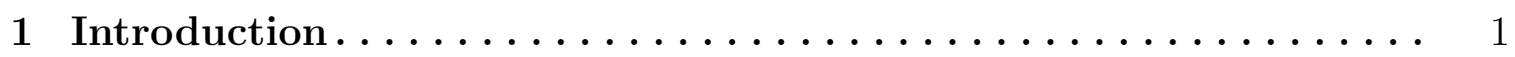

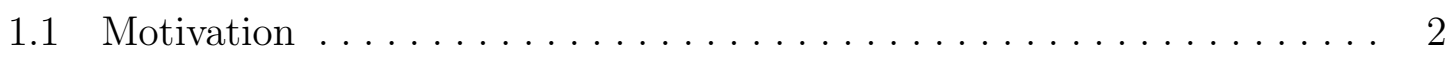

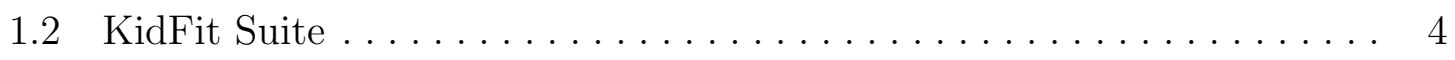

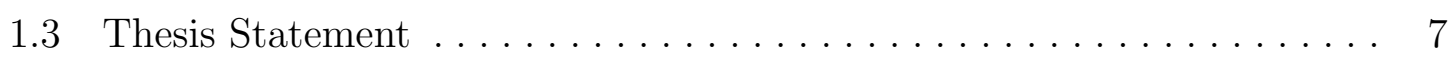

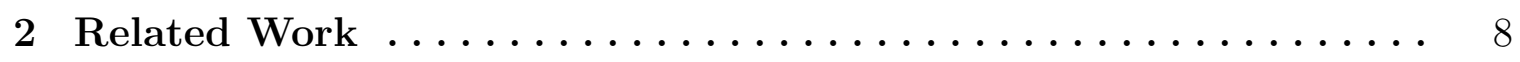

2.1 Understanding of Fitness Tracker Usage . . . . . . . . . . . . 8

2.2 Understanding Related Activity Games . . . . . . . . . . . . . . . 11

3 Investigating Children's Fitness Tracker Usage . . . . . . . . . . 14

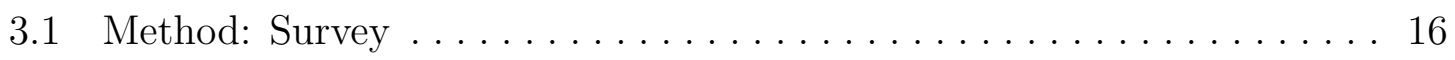

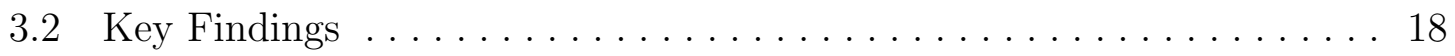

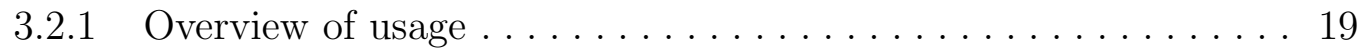

3.2.2 Reasons for adopting (or not) fitness trackers ........... 24

3.2 .3 How children use fitness trackers . . . . . . . . . . . 27 
3.2.4 Parents' perceptions of fitness technology . . . . . . . . . . . . 29

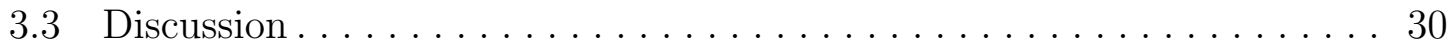

4 What Makes Physical Activity Fun ................. 35

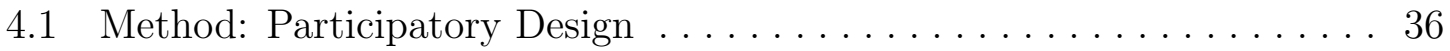

4.1 .1 Zombies Run! ........................ 36

4.1 .2 Virtual Walk . . . . . . . . . . . . . . . . . . 38

$4.1 .3 \quad$ NFL PLAY $60 \ldots \ldots \ldots \ldots \ldots \ldots \ldots \ldots$

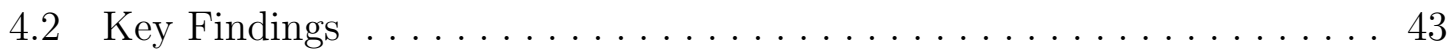

4.3 Discussion . . . . . . . . . . . . . . . . . . . . 44

5 Developing for Social and Temporal Impact . . . . . . . . . . . . 45

5.1 Key Elements in the KidFit Suite . . . . . . . . . . . . . . . . 46

5.1 .1 Social Interaction $\ldots \ldots \ldots \ldots \ldots \ldots$

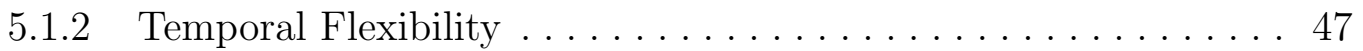

5.1 .3 Persuasive Display . . . . . . . . . . . . . . . . . . . . 48

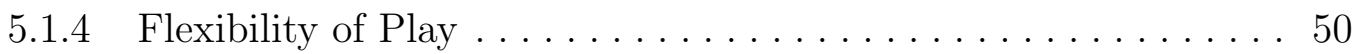

5.1 .5 Steps as a Measure of Activity . . . . . . . . . . . . . . . . 50

5.1 .6 Secure and Easy $\operatorname{Login} \ldots \ldots \ldots \ldots \ldots$. . . . . . . . . . 51

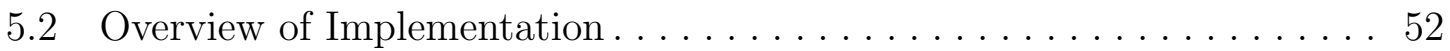

5.2 .1 MySQL Database . . . . . . . . . . . . . . . . . . . 52

$5.2 .2 \quad$ FitBit Data Access . . . . . . . . . . . . . . . . . 53

5.2 .3 KidFit API's . . . . . . . . . . . . . . . . . . . . . . . 57

5.2 .4 Data Flow . . . . . . . . . . . . . . . . . . . 57

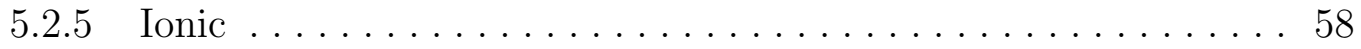

5.3 Game Walkthrough . . . . . . . . . . . . . . . . . 59 


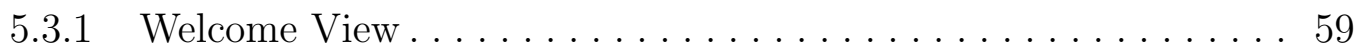

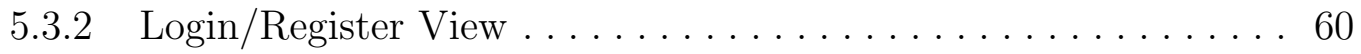

5.3 .3 Forgot Password View . . . . . . . . . . . . . . . 61

5.3 .4 Games View ......................... 61

5.3 .5 Create a Game View . . . . . . . . . . . . . . . . . . . 63

5.3 .6 Competitive Game View . . . . . . . . . . . . . . . . . 65

5.3 .7 Collaborative Game View . . . . . . . . . . . . . . . 66

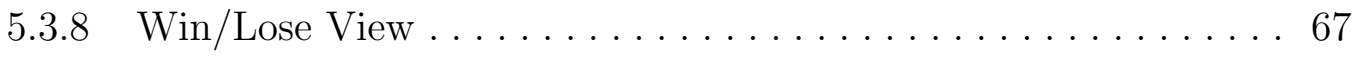

6 Investigating Social and Temporal Aspects ............ 70

6.1 Method: User Study . . . . . . . . . . . . . . . . . . . . 70

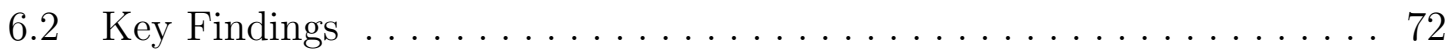

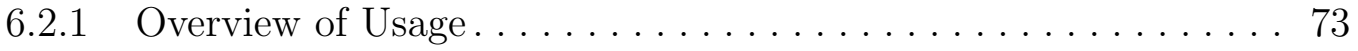

6.2 .2 Social Element and its Impact $\ldots \ldots \ldots \ldots \ldots \ldots \ldots \ldots$

6.2 .3 Temporal Flexibility and its Impact $\ldots \ldots \ldots \ldots \ldots \ldots \ldots$

6.3 Discussion . . . . . . . . . . . . . . . . . . . . . 91

7 Future Work \& Conclusion $\ldots \ldots \ldots \ldots \ldots \ldots \ldots \ldots \ldots \ldots$

7.1 Future Work . . . . . . . . . . . . . . . . . . . . . . 94

7.1.1 Simplify Activity Measures . . . . . . . . . . . . . . . . 94

7.1.2 Add a Medium of Communication . . . . . . . . . . . 95

7.1.3 Address Logging Out of the Application . . . . . . . . . . . . 96

7.2 Conclusions............................ 96

7.2.1 Steps Increased in G2, Activity Minutes Did Not . . . . . . . . 96

7.2.2 Visual Preference for CTC $\ldots \ldots \ldots \ldots \ldots \ldots \ldots \ldots . \ldots 7$

7.2.3 Motivation to Win Led to More Collaborative Games ..... . . 97 
7.2.4 Impact of Social Interaction: Collaborative Preferred . . . . . . . . 97

7.2.5 Impact of Temporal Flexibility: Synchronous Preferred . . . . . . 98

7.2.6 Social and Temporal Correlations . . . . . . . . . . . . . 98

7.2 .7 Overall Response . . . . . . . . . . . . . . . . . . . 98

7.3 Revisiting Thesis Statement . . . . . . . . . . . . . . . . . . . . . . . 99

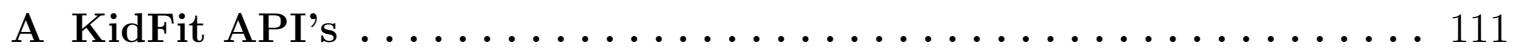

B Mechanical Turk Survey Questionnaire . . . . . . . . . . . . 117

B.1 Generic Questions Related to Parents . . . . . . . . . . . . . 117

B.2 Questions Related to Children's Fitness tracker usage . . . . . . . . . . 118 


\section{LIST OF TABLES}

3.1 Parents' perception related to younger children not needing a fitness tracker

3.2 Parents' response related to why they bought a fitness tracker for their

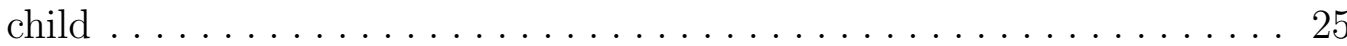

3.3 Parents' perceptions related to why children do not need a fitness tracker (besides being too young) . . . . . . . . . . . . . . . . . . 32

3.4 Parents' response related to how their children use fitness trackers . . . . 33

3.5 Parents' responses related to why would they buy a fitness tracker . . . . 33

3.6 Parents' responses related to why they acquired (or would want to acquire) a fitness tracker in order to bond with family . . . . . . . . . . 34

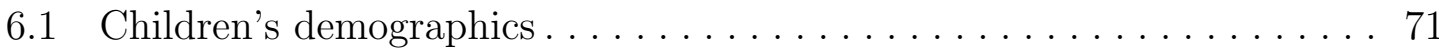

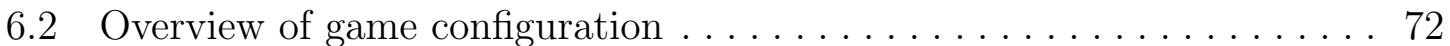

6.3 Overview of games played $\ldots \ldots \ldots \ldots \ldots \ldots \ldots \ldots \ldots \ldots \ldots$

6.4 Distribution of games played and activity collected by G1 . . . . . . . 74

6.5 Distribution of games played and activity collected by G2 . . . . . . 74

6.6 Children's activity data during the second week of the study $\ldots \ldots$. . 76

6.7 Distribution of games played on social and temporal preferences $\ldots$. . 77

6.8 Children's and parents' views related to the social element in the

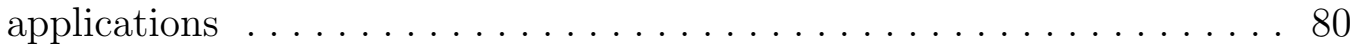


6.9 Children's and parents' responses related to who would they like to play with $\ldots \ldots \ldots \ldots \ldots \ldots \ldots \ldots \ldots \ldots \ldots \ldots \ldots \ldots \ldots \ldots$

6.10 Motivating group names created by children $\ldots \ldots \ldots \ldots \ldots \ldots 81$

6.11 Children's and parents' responses related to social motivation of the

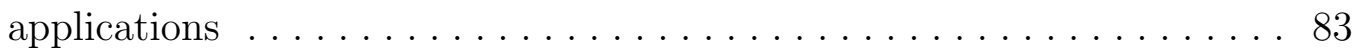

6.12 Children's and parents' responses related to collaborative game play . . 84

6.13 Children's and parents' responses related to competitive game play . . 85

6.14 Children's and parents' responses related to fewer GTG games . . . . . 87

6.15 Children's and parents' suggestions related to streamlining game invi-

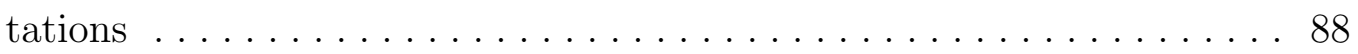

6.16 Children's and parents' responses related to temporal Flexibility in the applications $\ldots \ldots \ldots \ldots \ldots \ldots \ldots \ldots \ldots \ldots \ldots \ldots$

6.17 Children's and parents' responses related to short synchronous games. . 90

6.18 Children's and parents' responses related to long asynchronous games . 91 


\section{LIST OF FIGURES}

3.1 Fitness tracker usage among children by age. . . . . . . . . . . 20

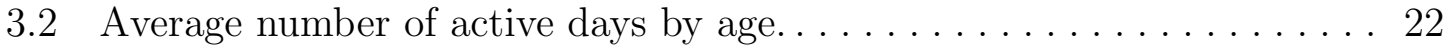

3.3 Screen time reported by parents for children. . . . . . . . . . . . 23

3.4 BMI percentile distribution in younger (left) and older (right) children. 24

3.5 Fitness tracker usage in younger and older girls (left) and boys (right). 24

3.6 Parents' perception related to activity induced by fitness trackers in children $\mathrm{T}_{\text {current }}$ and $\mathrm{T}_{\text {past }} \ldots \ldots \ldots \ldots \ldots \ldots \ldots \ldots \ldots \ldots \ldots \ldots \ldots \ldots \ldots$

3.7 Average number of active minutes per day, as calculated by activities (e.g. walking, cycling), the number of days each activity was performed, and the daily duration of each activity as reported by parents in the last 7 days. . . . . . . . . . . . . . . . . . . . . 29

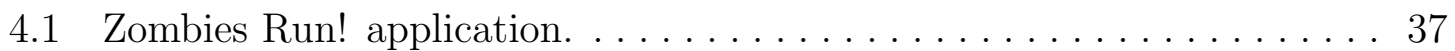

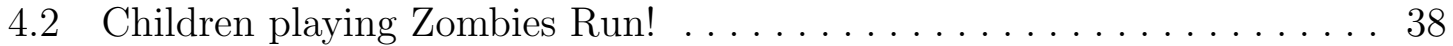

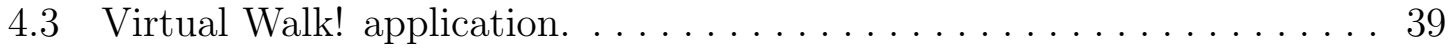

4.4 Children playing Virual Walk . . . . . . . . . . . . . . . . . . . 40

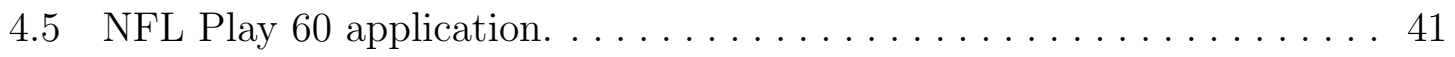

4.6 Children playing NFL Play $60 \ldots \ldots \ldots \ldots \ldots \ldots$. . . . . . . . . 42

4.7 Children's ratings of Zombies Run!, Virtual Walk and NFL Play 60 . . 43 
5.1 Data Flow between the application, back-end API's, Databse and Fit-

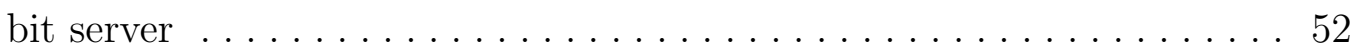

5.2 Entity Relationship diagram of KidFit Database . . . . . . . . . . . . . 54

5.3 Welcome view . . . . . . . . . . . . . . . . . . . . 59

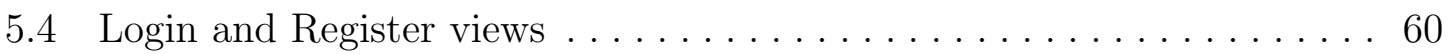

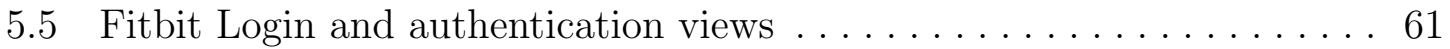

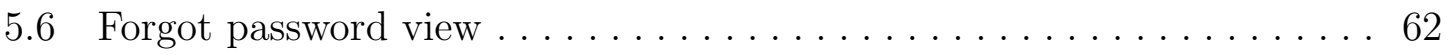

5.7 Games view (GTG on the left; CTC on the right) . . . . . . . 63

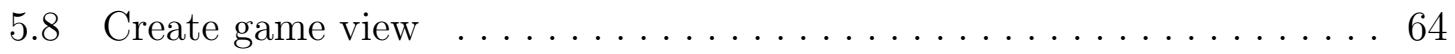

5.9 Created game view (left); Remove a player (right) . . . . . . . . 65

5.10 Ready to play Game view $\ldots \ldots \ldots \ldots \ldots \ldots$

5.11 Competitive game view GTG on the left \& CTC on the right $\ldots \ldots 67$

5.12 Collaborative game view GTG on the left \& CTC on the right $\ldots \ldots 68$

5.13 Win game view GTG on the left \& CTC on the right $\ldots \ldots \ldots \ldots 8$

5.14 Lost game view GTG on the left \& CTC on the right . . . . . . 69 


\title{
LIST OF ABBREVIATIONS
}

\author{
API - Application Programming Interface \\ CHI - Computer Human Interaction \\ HCI - Human Computer Interaction \\ IRB - Institutional Review Board \\ NPM - Node Package Manager \\ PHP - PHP HyperText Preprocessor \\ RDBMS - Relational Database Management System \\ UI - User Interface \\ URL - Uniform Resource Locator \\ XML - Extensible Markup Language
}




\section{CHAPTER 1}

\section{INTRODUCTION}

With the ubiquitous spread of technology, computers and mobile phones have become an integral part of modern life for both adults and children. Children spend a considerable amount of time using such technologies. In addition, children worldwide spend an average of 8.6 hours daily in sedentary time and $54.2 \%$ of children fail to meet screen time guidelines [39]. The overuse of technology is sometimes blamed for the shift to a more sedentary lifestyle. This change in lifestyle is believed to be

one of the causes of the obesity epidemic in children. Several technologies including fitness trackers, exergames, and fitness games motivate young and old to be physically active. This research investigates the effectiveness of the social and temporal factors in fitness games for children. As a part of this research, several mobile applications were developed to investigate the impact of these factors. These applications aim to encourage physical activity by providing a platform to enable social engagement via competition and collaboration and temporal engagement via synchronous and asynchronous types of play. This chapter presents additional motivations for this work and an introduction to KidFit Suite (see Section 1.2). 


\subsection{Motivation}

Obesity is a condition of having excess body fat. Childhood obesity is a growing cause of concern especially in the United States. Nearly one fifth of the children in the United States are affected by the chronic disease of obesity [10]. The physical activity guidelines for children suggest 60 minutes of activity daily. However, only $21 \%$ of American youth meet this guideline [50]. Another recent study shows there has been a tenfold increase in obesity in children and adolescents in the last four decades [51]. Obesity not only affects physical health but hampers the overall social and emotional well-being of a child. Other health complications related to obesity include increased risk of premature onset of illness like diabetes and heart disease. Obesity in children has a higher chance of persisting into adulthood and increases the risk of related health issues [27].

In order to combat the change in lifestyle towards sedentary behaviors and the increase in overweight and obese children, research has explored utilizing technology as a bridge to engage individuals in physical activity $[44,38,21]$. Some of these activity enhancing technologies include fitness trackers, fitness mobile applications, virtual reality games, applications with haptive feedback based on the activity level of the user and exergame applications.

Fitness trackers are modern day upgrades of a pedometer that lets users track their steps and activity levels, but also offer features such as measuring sleep, flights of stairs, hourly activity during the day, number of days exercised in a week and more. The idea is to make users aware of their goals and their current activity levels. Exergames are interactive video games that employ elements of gaming to engage users into physical activity. Smartphones have emerged as a way to track 
activity and engage people in fitness routines using a variety of applications that are available to almost everyone. More than half of smartphone users have a health app installed [62]. Fitness mobile applications may use awareness or gamification or both to motivate users to be active. Nike Training app is one such application that utilizes awareness while PokemonGo uses gamification.

While there are plenty of technologies available to promote physical activity in individuals, most of these devices or applications are designed and developed targeting adults as their primary users, though some devices target children as their primary users. Children, however, tend to follow their parents or guardians and usually adopt technologies used by them as was observed in a survey on children's fitness tracker usage conducted by me (See Chapter 3). As the physical activity guideline for children suggests activity in terms of active minutes, designing technology that addresses this guideline would be better suited for children. When designing technology for children, designers should address their needs according to their age, gender, and overall development. It should not be complex to learn or use and easy to perceive and understand for a child [54]. An important design guideline when designing fitness technology for children is that it should not convey a negative body image (e.g. the concept of being overweight or obese) to children and should, instead, aim at engaging children in physical activity in a fun and playful manner [54].

Due to the increasing awareness of the need to encourage physical activity in children, companies like FitBit, Garmin, Vtech and others have designed fitness trackers especially suited for them. Fitness Games like Bunny Bolt, Virtual Walk, and NFL Play 60 were developed to promote physical activity in children. As an example, 'Make fitness fun for kids' is the tagline of the new FitBit Ace designed specifically for children which illustrates an emphasis of correlation of fun with fitness 
to further motivate children to be active. While fitness trackers promote activity by making users aware of their activity, it is advised to encourage activity in children by not focusing on the need to exercise [31], it should be on play. Physical activity induced by technology in children should be in fun and playful manners, which are characteristics of childhood play [58].

In previous research led by me, three elements were found that made fitness applications fun and playful for children (Chapter 4). These include social interaction, narratives and the flexibility to add their own elements [58]. Learning from this and other research, I developed KidFit Suite, a collection of mobile applications which is utilized as a tool to understand the effect of social and temporal aspects of fitness games on children's physical activity.

\section{$1.2 \quad$ KidFit Suite}

KidFit Suite is a collection of mobile applications and back-end API's that fetch fitness data from the FitBit server and present it to the user in gaming applications. The gaming applications leverage children's affinity towards technology and mediums of social interaction and temporal flexibility to engage users in physical activity. It employs the elements of fun with fitness, flexibility while playing the game, interaction with peers and a narrative to motivate children towards activity which were found to be preferred by children [58]. These applications are targeted towards an audience in the age-range of children 6-11. However, adults can also play with children and make physical activity something that the entire family can do together. The applications present you with data in both steps accumulated and activity minutes so as to make it suitable for both adults and children. 
KidFit Suite consists of the three games Grow the Garden, Capture the Crown and Hot Potato. These gaming applications utilise elements of social collaboration, flexibility of play timing and persuasive display to encourage children to be physically active. They constantly communicate with the KidFit server API's to fetch details from the database and the Fitbit server. The data fetched from the KidFit API's include details about the user, their activity details, their games and the activity accumulated in those games. Following is an overview of how each game works:

- Grow the garden (GTG): This application displays children's activity by adding flowers to the garden on their screen as they accumulate activity similar to [22]. The users can choose to challenge their peers where each individual competes and the one who achieves maximum activity at the end of a timer, wins the game. This game can also be played as a team, where based on the number of users, the application provides a target number of steps or activity minutes to achieve. As the total activity by all the players increase, flowers start showing up on the screen one by one notifying the user of the progress so far. If the group of users are able to achieve the target provided by the app, the team wins. The applications involve both synchronous and asynchronous types of play. In synchronous play, the users all play at the same time and the duration of the game is usually small. In contrast asynchronous play lets children play as and when they are available and the duration of the game is longer. This provides temporal flexibility and the users can play at their own time and pace.

- Capture the crown (CTC) Similar to Grow the garden, Capture the crown uses persuasive display in which users earn jewels on their crown as they achieve 
activity and proceed through the game. Capture the crown also supports competition and collaboration. Games can be played synchronously or asynchronously.

- Hot potato As the name suggests hot potato is game where children take turns while playing and is a competitive game. As the game starts, the application randomly assigns a hot potato image to one of the players, that player will now have to achieve a certain amount of activity to pass the potato to the next player. Similarly, the same amount of activity lets the other player pass the potato to a next player and so on until a random timer fires. At the end of the timer, the user having the potato loses the game while everyone else wins. Unlike the other two games, this game uses negative reinforcement of selecting one loser and the rest all winners while in the other games, there is positive reinforcement i.e. one winner and the rest losers.

The applications in KidFit Suite try to incorporate the elements of social interaction, temporal flexibilty, visual persuasion and flexibility of play to motivate children to be physically active. KidFit Suite combines characteristics of childhood play of playing with friends and siblings to reduce the sedentary time using technology and convert it into active play. Children have the flexibility to play short synchronous or long asynchronous games. Unlike applications like PokemonGo which are designed for adults and require an individual to go out in order to collect pokemons, these games can be played indoors or outdoors depending on the context and weather. The persuasive displays of collecting flowers or jewels is easy to understand and visually promising as a user can see their progress during the game.

The KidFit API's facilitate storing and retrieving user information from a database. The mobile applications communicates with API's to enable log-in, fetching data, 
password reset, the ability to create new games and visualize their progress during a running game. This combination of the API's and gaming applications encourages a child to be active with their friends or family and can be utilized when it suits their schedule.

\subsection{Thesis Statement}

This research seeks to better understand the factors that contribute to making physical activity fun and playful for children. Initial findings from Chapter 3 and Chapter 4 informed the design of KidFit Suite. The applications in KidFit Suite are utilized to investigate the effect of the following dimensions on children's physical activity:

- Social Interaction - Competitive versus Collaborative Experiences: A competitive game lets the children compete against each other and a collaborative game lets children play as team.

- Temporal Flexibility - Synchronous versus Asynchronous Experiences: A synchronous game is usually a short game and expects all the users to be active at the same time for the duration of the game. An asynchronous game on the other hand is longer and the users can be active intermittently.

This research seeks to understand how these dimensions impact children's physical activity and engagement. 


\section{CHAPTER 2}

\section{RELATED WORK}

This chapter situates my research in the context of related work, by first sharing information about fitness trackers and the surveys exploring their usage. The following section will discuss the prior research in fitness games for children and how this research fits among the existing work.

\subsection{Understanding of Fitness Tracker Usage}

A number of surveys have examined the perception and use of fitness trackers by adults. Not surprisingly, fitness tracker use is higher for those who perceive trackers as leading to better health outcomes [45]. Adults adopting fitness trackers perceived them as both useful and easy to use [45]. A survey of Australian adults conducted in 2014 found that $35 \%$ were using or had used an activity tracker, $16 \%$ were interested in using one, and $44 \%$ were not interested [13]. Males, non-workers, those with lower education levels, and inactive participants were significantly less likely to have used a fitness tracker. Interestingly, of those who had used a tracker, only $48 \%$ believed the tracker helped them get more active, while $50 \%$ believed it did not help. The primary reasons for not wanting a fitness tracker were: (1) they did not want to increase physical activity, and (2) they did not think a tracker would be helpful, which were consistent with surveys in other countries $[36,59]$. Other factors like cost, 
technology, lack of time, being too old, health, and having no interest were much rarer. Privacy concerns were rarely raised in these large surveys, although they were the focus of another survey that helped illustrate the most common concerns related to data privacy [43]. Although results from such surveys are useful in characterizing adults' use and attitudes toward fitness trackers, general surveys about children's experiences are absent in the literature.

A different strand of research has examined the behavior change techniques used in fitness trackers. Studies such as Duncan's et al., from 2017 [25] characterize the behavior change techniques embedded into fitness trackers, which primarily center around self-monitoring and self-regulation, as opposed to techniques related to planning or instructions [48]. However, children differ significantly from adults [17], making it unclear how well such behavior change techniques will appeal to children. For example, a focus group study of Australian teens showed mixed feelings about the desire to have a set goal per day or receive achievement badges [56]. Furthermore, it is not always clear if parents or children or other adults should set the goals [55]. Although several health-focused studies have examined fitness tracker interventions for children and youth (see [55] for a literature review), they are often conducted with a unique subset of youth (e.g., leukemia patients [33]) or a unique setting (e.g., school environment $[41,42])$. It is not clear that the short-term benefits observed in these settings will extend to more common usage scenarios and contexts.

Other studies have focused on the usability of fitness trackers among adults and occasionally children. For example, a study of those who abandon personal health-tracking technologies identified three design opportunities for fitness trackers including: self-monitoring as a means of supporting a variety of goals, providing more robust analytics of the data, and supporting social interactions around fitness data 
[19] which is more pertinent to my research. Such design ideas may apply to children, though they would likely lead to very different designs focused on, for example, family social dynamics.

Another study of adults identified the importance of several design aspects for trackers including the appearance of the wearable, wearability (e.g., implications of having a wrist-based wearable; concerns about damaging the device), intensity of interaction (e.g., vibrations for notification; visual display of progress), activity measures, and validity of data [49]. It is likely that many of these factors will also affect children, though the preferences of children and adults for various designs (e.g., colorful or thematic fitness trackers) may differ substantially. Other social and environmental factors may also be critical to children that have not yet been explored sufficiently; for example, a study of the impact of socio-economic status on activity tracker use by youth found that the attitude of the caregiver and safety of the neighborhood affected use [57]. Other recent work suggests that parenting practices regarding physical activity are affected by technology [53].

While they are relatively sparse, a few articles have directly addressed the potential challenges of children using the current, adult-focused fitness trackers. One focusgroup study of teens identified barriers such as a lack of knowledge of how to use the device, inability to wear it for certain sports (in order to reduce injuries), forgetting to wear it, and a lack of desire after the novelty wore off [56]. Another study examining the psychological effects of fitness trackers on youth showed that short-term increases in physical activity were due to feelings of guilt, competition, and internal pressure, while over time the fitness tracker actually led to less motivation to exercise [37]. Medical professionals have also warned parents against the potential of fitness trackers in promoting "compulsive exercise" (e.g., preoccupation with weight and exercise; 
feeling guilty when not able to exercise), as well as the practicalities of younger children losing, breaking, or forgetting to charge their fitness tracker, or not having a

phone to sync it with $[5,6]$. However, research has not substantiated these concerns or articulated how prevalent they are among parents and children.

Considerable research has examined the use, effectiveness, and perceptions of fitness trackers among adults, with only a few studies focused on youth. There are likely significant differences in how fitness trackers can lead to behavior change in adults and children as children have different goals, interaction styles, motivations when they engage with such technology. Likewise, there are likely different benefits and challenges faced by adults and children. While a handful of studies have used focus groups or pilot studies to explore youth perspectives on fitness trackers, many are focused on specific environments (e.g., school [41, 42]) or groups (e.g., leukemia patients [33]).

\subsection{Understanding Related Activity Games}

Mobile devices have become ubiquitous and mobile applications are used by one and all. Several mobile applications which encourage users to be physically active have been researched. PokemonGo is one such mobile gaming application which became a phenomenon soon after its release in 2016 [15]. This application presents its users with a virtual world of Pokemons where walking in certain directions lets you catch one. The applications expects a user to explore their outdoors while playing and being active. Zombies Run!, is a similar application where players walk and run to escape a zombie apocalypse. Other such games include Missile War, Charity Miles, and GeoCaching $[20,52]$. These applications utilize activity awareness and elements 
of game play to motivate and engage their users. Most of these applications are designed for adult users, and may or may not be suitable for young children. However, there are a few applications designed specifically for children. Bunnybolt [38] is one application intended to be used by children and utilizes a youth friendly storyline to engage them into short activity sessions. This application intends to leverage the engagement brought about by video games that encourages children to explore their outdoors. FitQuestLite [1] is another narrative based application where users activity helps a baby squirrel safely reach the tree house. Other examples include NFL Play 60 and Virtual Walk. These applications are intended to be used by children and hence employ child-friendly narratives. Children engage in activities that focus more in recreation than education [31], hence narratives seem to be an appropriate gaming mechanisms to promote activity in children.

A visual display is often used as a tool of effectively communicating information especially with children. Several health-related mobile applications use persuasive displays as a means of improving activity awareness in the users. While some mobile applications inform users of their sedentary screen time others increase awareness by informing users of their current activity levels. Ubifit [22] is one such example where users are presented with a visual display of a garden. The garden display blooms based on user's activity through the day. The awareness and motivation brought about the garden not only helped users meet their daily activity goals but were found to maintain their activity levels over time, even during holidays [22, 21].

Similar visual persuasion was utilized by Fish'n'Steps [44] which imitates user activity by growth and encouragement of an animated virtual pet fish. The results showed that the awareness brought about by the Fish helped individuals incorporate activity in the daily routine [44]. The study also compared the effects of social 
components of collaboration and competition but the participants had no choice in the type of social interaction. Neither of them had any choice of whom and how long they want to play with. Social elements of competition and collaboration have been found to impact physiological and psychological behaviors [46]. Competition has been found to promote energy expenditure in exergames while collaboration increases motivation [46]. FitPlay games [60] is a project that presented users with competitive asynchronous games. The element of competition and temporal flexibility was liked by the participants however their average steps during the study did not increase. They used Fitbit data to measure activity and the results were reported based on steps taken every hour while the app was installed on the users' phone.

The applications mentioned above use one or more of the elements of gamification, short sessions of activity, persuasive displays, temporal flexibility, social elements of cooperation and collaboration, and narratives to engage children in physical activity games. Though these applications highlight the various elements that helped or may help motivate individuals to be physically active, most of them have been researched with adults. Understanding how children react to these elements needs to be explored further. As each child interacts with technology differently based on the age, gender or surrounding environment, a single element might not be enough to encourage them to be active. KidFit Suite combines the elements found from aforementioned research (social interaction, temporal flexibility, narratives, persuasive display) and presents them as one application. The applications in KidFit Suite are utilized to evaluate the social and temporal aspects in fitness games on children's physical activity. 


\section{CHAPTER 3}

\section{INVESTIGATING CHILDREN'S FITNESS TRACKER USAGE}

Sedentary behaviors deter an individual from physical activity [35]. Sedentary behaviors are sometimes linked with aggressive behaviors, negative body images, unhealthy eating habits, and obesity [35]. Technology is sometimes blamed for this increase in sedentary time and health issues that arise from that inactive time. The guidelines for children and youth recommend 60 minutes of activity per day [2]. However only $21 \%$ of American youth meet these guidelines [50]. In fact, more than $18 \%$ children in the United States are obese [10]. In response to these issues, a wide variety of wearable fitness technologies are available for both adults and children including standalone fitness trackers (e.g., FitBit, Jawbone), smart watches and mobile phone fitness applications that track activity via accelerometers or GPS. These modern incarnations of pedometers have become quite popular in recent years. Over 87 million standalone fitness trackers were sold in 2017 with an additional 75 million smartwatches, nearly all of which include built-in fitness tracking [7]. Moreover, more than $50 \%$ of smartphone users have a health app installed [62], in addition to the built-in Apple Health app (on all iOS devices) and Google Fit app (on most Android devices) that track activity.

Fitness trackers and their compatible mobile and smartphone apps provide several 
features. At their core, they track step counts, distance covered, active minutes, calories burned, and potentially other measures like heart rate, flights of stairs and progress toward goals. Most recent versions let you share your data with friends, set goals, participate in challenges and measure hours and quality of sleep [25]. The majority of trackers have been designed for adults, without having youth or children in mind [19]. This is discouraging given that children have different physical, social, and emotional needs than adults which need to be addressed in the design of technology for children. However, things are beginning to change. Several companies have released fitness trackers designed for children like Vivo, Garmin and Fitbit. No studies have examined these new child-specific devices yet, and only a couple have investigated trackers for children $[19,55]$ and are discussed below.

Studies are mixed on the effectiveness of current fitness trackers as a behavior change intervention designed to increase physical activity. Controlled studies of adults show that the most common behavior change techniques used in fitness trackers include self-monitoring and self-regulation, both of which are effective for adults [25]. However, approximately one third of all adults quit using fitness trackers within 6 months in the wild [40]. Supporting social interactions around activity tracking was also seen as something that can promote activity [19]. Unfortunately, there is a "paucity of research concerning the effectiveness and feasibility of wearable activity trackers as a tool for increasing childrens and adolescents physical activity level" as one recent systematic review from 2016 concluded [55]. While some short-term interventions that incorporated fitness trackers showed promise, it is not at all clear that techniques that work for adults will also work for children (e.g., goal setting and monitoring) [55]. Indeed, one cautionary study of youth age 13-14 who were given fitness trackers showed that initial increase in motivation were based on competition, 
guilt, and internal pressure transitioned into increased amotivation and decreased autonomous motivation [37]. These negative effects may be due to a lack of child-centric designs and behavior change strategies as described above.

In order for fitness trackers and corresponding behavior change interventions to be designed well for children, we must gain a better fundamental understanding of how parents and children perceive fitness trackers and what are their goals or objectives as they interact with the technology. Children's understanding of the need of activity and role of activity trackers in making an individual active also plays a role in the adoption of technology. To help better understand these issues, I conducted a survey of 98 parents recruited via Amazon's mechanical Turk. The next section discusses my research in the context of related literature about fitness trackers for children. The survey method and the participants are described more specifically in the following section.

\subsection{Method: Survey}

In order to address the lack of understanding of children's fitness tracker usage, I designed a survey that examines the use of trackers by children as reported by their parents. 98 parents reported on a total of 113 children (ages 6 to 11), answering questions about children's (and their own) use and perception of fitness trackers. Out of the 113 children, 59 were girls and 54 were boys. All the participants of the survey reported to be from the United States. To simplify the presentation of information, children are divided into two groups: younger children ages (6-8); and older children ages (9-11).

Our survey focused on preteen children (ages 6-11) from the perspective of a 
parent.The survey was conducted using Amazons Mechanical Turk (MTurk). The survey was only taken by the workers with "Master" status who are identified as high-performance workers by MTurk analyses thereby increasing the reliability of the workers. The screening criteria for the survey was parents who had one or more children (ages 6-11). The consent form indicated that the parents could participate in the survey even if their children did not use a fitness tracker (and indeed $40 \%$ of the children reported on in this survey had never used a fitness tracker). The average time to complete the survey was a little over 13 minutes and participants were compensated.

The survey included questions related to children's demographics, quantitative questions relating to their activity and fitness tracker usage, and qualitative questions relating to parents perceptions about such technology, its usage and impact on children (see Appendix B). I also investigated the perceptions that parents have about the effectiveness of these trackers in motivating children to be physically active. The responses to the questions provided insight into why parents and children acquire, use, or stop using fitness trackers and parents perceptions about childrens interaction with fitness technology if their child currently uses, has used in the past but is not using currently, or never used a fitness tracker. Parents were asked whether or not they used a fitness tracker ( $80 \%$ of which did) and then asked specific questions about each of their children between the ages of 6 to 11. Demograhic questions included the child's age, gender, height and weight, their physical activity, whether or not they use (or have used) a fitness tracker, what kind of tracker they use, how they use it, and other qualitative questions to provide additional insights about how a fitness tracker may or may not motivate their child to be more active.

In this chapter, I present the findings from the quantitative and qualitative answers 
to the survey and discuss relations found between childrens demographics, fitness tracker usage, and parents perceptions. Patterns relating childrens demographics, parents perception, and activity levels to better understand the impact of fitness tracker technology in promoting physical activity in children are also presented. To understand the relationships between our quantitative data, I used the graphlab library [28]. The qualitative answers were first classified into broad categories and codes were refined iteratively $[22,23]$, and were later used by three researchers to independently code the qualitative responses of the survey. Cohens Kappa was utilized to calculated inter-rater reliability. The Cohens Kappa was calculated individually for each question and ranged from 0.92 to 1.0 signifying a high degree of agreement between the three raters. In the next section, I will elaborate on the key findings from the survey and their correlation with previous research.

\subsection{Key Findings}

This section discusses the findings from this survey in four major categories based on the usage of children as reported by parents. The categorization is based on the questions and parent's responses. The categories are: overview of usage (Section 3.2.1), reasons for adopting (or not) fitness trackers (Section 3.2.2), how children use fitness trackers (Section 3.2.3), and parents' perceptions of fitness technology (Section $3.2 \cdot 4)$

Some overviews are given by individual age (ages 6-11) to show general trends, however in most cases, to simplify the representation of data, children are categorized into two age groups: younger children (ages 6-8) and older children (ages 9-11). Based on their fitness tracker usage, children were further divided into three groups: those 
who have never used a fitness tracker $\left(\mathrm{T}_{\text {never }},\left|\mathrm{T}_{\text {never }}\right|=50\right)$, those who have used a fitness tracker in the past but are no longer using one $\left(\mathrm{T}_{\text {past }},\left|\mathrm{T}_{\text {past }}\right|=25\right)$, and those who are currently using a fitness tracker $\left(\mathrm{T}_{\text {current }},\left|\mathrm{T}_{\text {current }}\right|=38\right)$. Out of the 113 children, 44\% (50 of 113) had never used a fitness tracker, 22\% (25 of 113) children had used a fitness tracker in the past but are no longer using one, and 34\% (38 of 113) children were currently using a tracker. Based on the age, weight, height and gender of the child as reported by their parent, I calculated the BMI percentile using $[4]$.

\subsubsection{Overview of usage}

This section presents an overview of children's fitness tracker usage. The data from our survey indicates an increasing trend of fitness tracker usage by age (see Fig 3.1).

Several reasons were provided in qualitative responses that helped explain reasons for this trend. Often, parents indicated that their child was "too young" for a fitness tracker and indicated one reason being that they did not feel it was age appropriate. Since the parent is likely the one who would purchase the tracker this could impact whether or not the child has a tracker. Responses to other questions indicated more specific reasons why some parents did not believe a fitness tracker was age appropriate (see Table 3.1). A higher percentage of older children using a fitness tracker could be due to increased adoption of technology as children get older [16]. One reason could be that most fitness trackers display more comprehensive data information through a mobile application which again may be more commonly used by older children. Another contributing factor to the higher use of fitness trackers in older children could be that parents are the primary instigators for getting younger children's fitness 


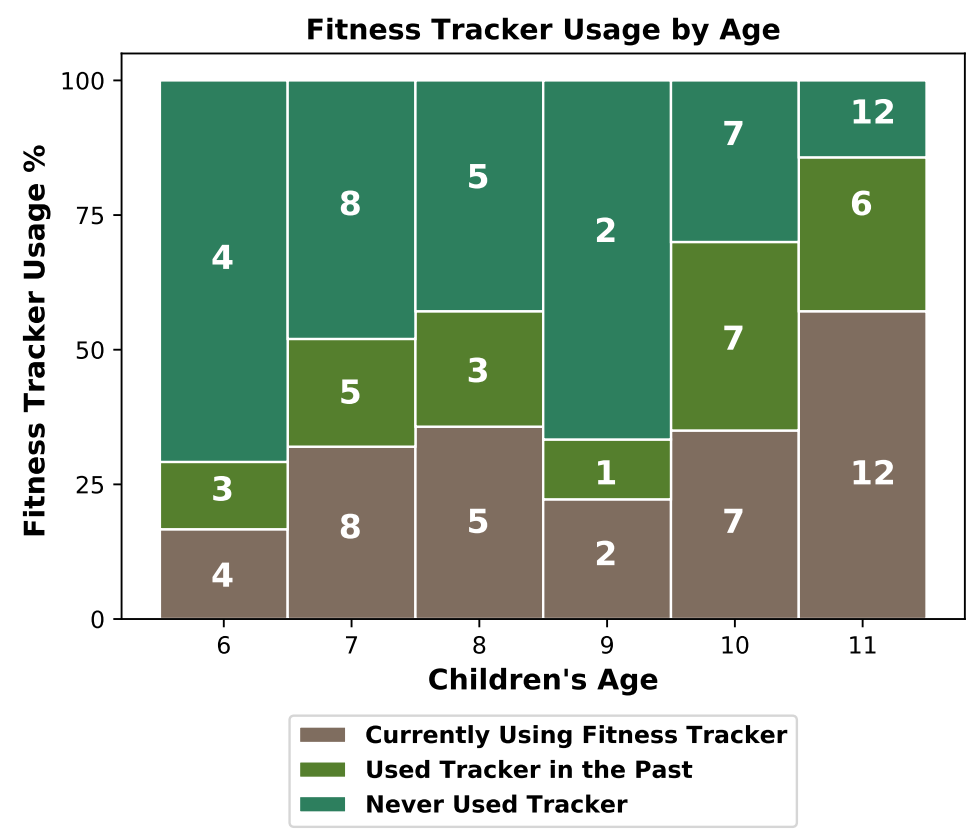

Figure 3.1: Fitness tracker usage among children by age.

trackers whereas older children are the primary instigators of getting a tracker for themselves. In fact, our data suggests that $70 \%$ of children who asked for a fitness trackers were older. As the qualitative responses to our survey indicate, parents sometimes do not trust young children with fitness tracker devices as they may break or lose them (highlighted in Table 3.3). One of the responses also mentions the pressure it can put on a child (P83). Some of the responses from parents are listed in Table 3.1.

Figure 3.2 shows the average number of active days (in the last week) by age (see B for details). The number of active days were reported by parents based on their perception of children's activity. As can be seen from the graph, the average number of active days seems to have a decreasing trend with age. The influence of school times, homework assignments, and extracurricular activities may 
Table 3.1: Parents' perception related to younger children not needing a fitness tracker

\begin{tabular}{ll}
\hline P3 & I don't think its [sic] necessary for a girl of that age \\
\hline P35 & I don't believe he is old enough to take care of the device properly. \\
\hline P40 & she is young \\
\hline P73 & $\begin{array}{l}\text { I don't think they are old enough to use that kind of technology } \\
\text { and really understand it yet }\end{array}$ \\
\hline P82 & Too young \\
\hline P83 & too young in my opinion and too much pressure \\
\hline
\end{tabular}

contribute towards lower activity times in older children. This decrease in average number of active days could also be a motivation behind parents reconsidering to buy a fitness tracker for them.

Parents were also asked about the average amount of screen time their children had each day. Screen time was defined as the time spent looking at computer screens, mobile phones, tablets, video game consoles, or other such displays. As illustrated in Figure 3.3, screen time tends to increase as children increase in age. This is consistent with the fact that older children use technology more than younger children and sometimes even have their own personal tablet or mobile device [16].

The healthy range for BMI percentile for children ranges from the 5th to the 85th percentile [4]. Even though older children had higher screen times and averaged less active days in a week, the BMI percentile in older children who use a fitness tracker were mostly in the healthy range (see $T_{\text {current }}$ in Figure 3.4, right). On the other hand, despite having a higher number of average active days and lower screen times, the BMI percentile in younger children who use a fitness tracker tends towards being overweight (see $\mathrm{T}_{\text {current }}$ in Figure 3.4, left). This differs from the common perception that 


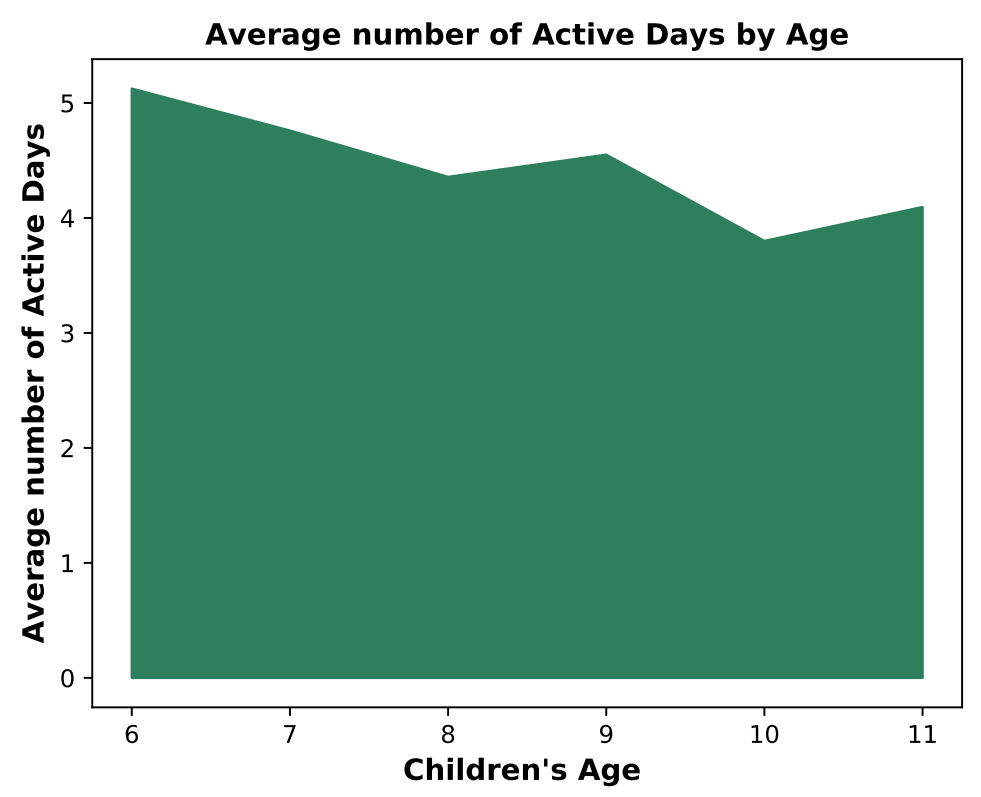

Figure 3.2: Average number of active days by age.

increased screen time negatively impacts BMI percentile. Our data also indicates that around $59 \%$ of young children who currently use a fitness tracker $\left(\mathrm{T}_{\text {current }}\right)$ were obese/overweight (above the 85th percentile BMI), based on the BMI calculated from the age, weight, height and gender reported by parents [4]. While not explicitly mentioned in the qualitative responses for these parents (except for 1), one reason for acquiring a fitness tracker for younger children may be because of their higher BMI. In response to why the parents would not consider buying a fitness tracker for their child, a few of them indicated that they did not think their children needed a fitness tracker because they were already physically active enough.

From the survey data, it was also observed that fitness tracker usage significantly increases $(20 \%$ to $50 \%)$ as girls age (see Fig 3.5 , left). In contrast, fitness trackers usage drops (40\% to $29 \%)$ for boys as they age (see Fig 3.5 , right). In contrast, several parents $(60 \% ; 15$ of 25$)$ indicated they 


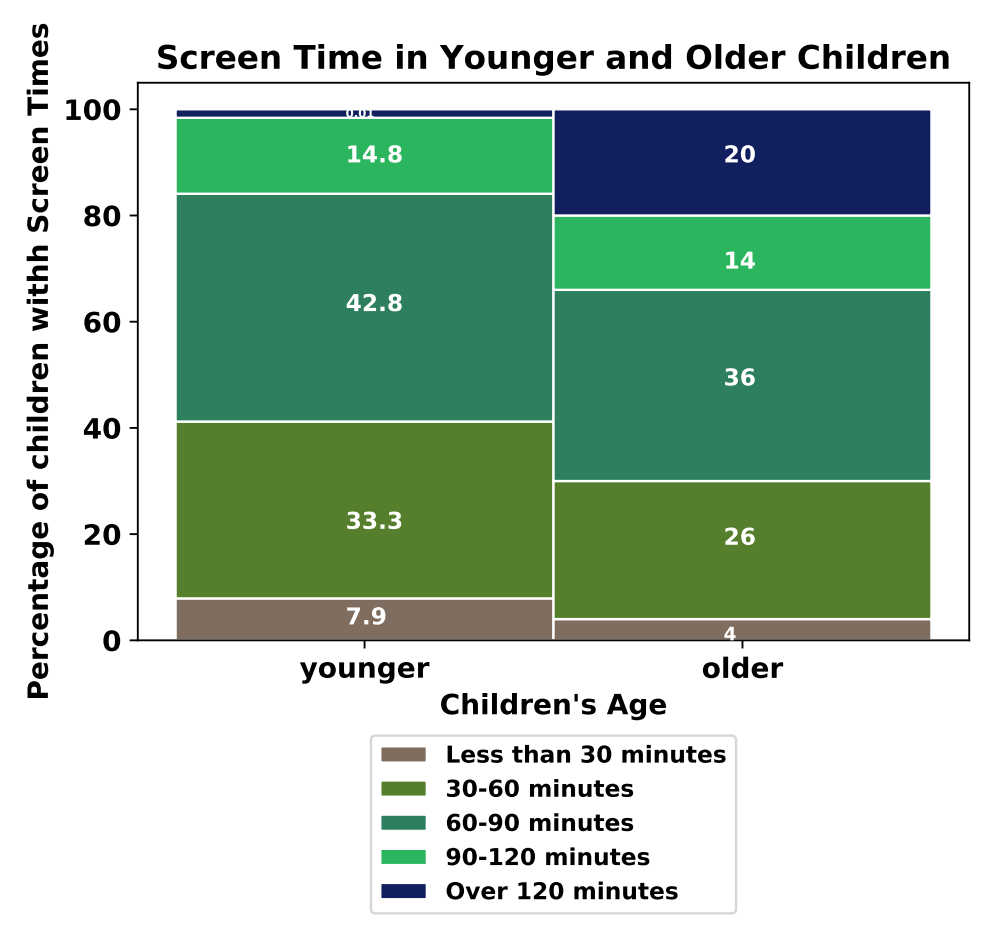

Figure 3.3: Screen time reported by parents for children.

stopped using a tracker because it did not make their child any more active and $36 \%$ of parents (9 of 25) observed that the primary reason children stopped using a tracker was they "got bored with it" or "lost interest". Other reasons included: losing the tracker $(12 \% ; 3$ of 25$)$, it did not fit well or was large in size (12\%; 3 of 25$)$, they forgot to charge it $(4 \% ; 1$ of 25$)$, they did not understand what it was supposed to do $(4 \% ; 1$ of 25$)$, and they did not buy a new battery $(4 \% ; 1$ of 25$)$. Some of these reasons align with the reasons mentioned by [56] where they noted similar barriers for using fitness trackers: lack of knowledge, forgetting to wear it, and lack of desire (getting bored) after the novelty wears off. 

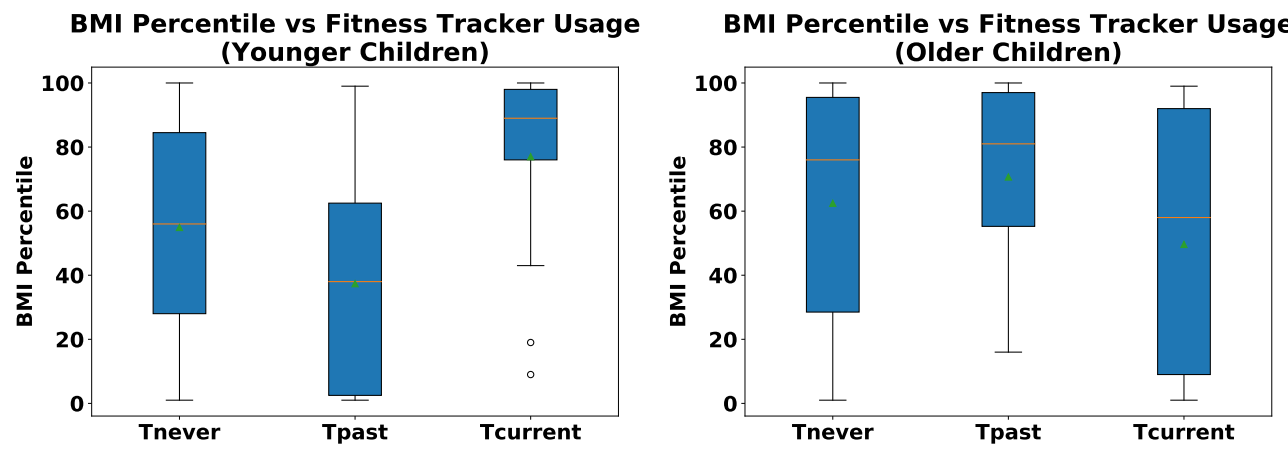

Figure 3.4: BMI percentile distribution in younger (left) and older (right) children.
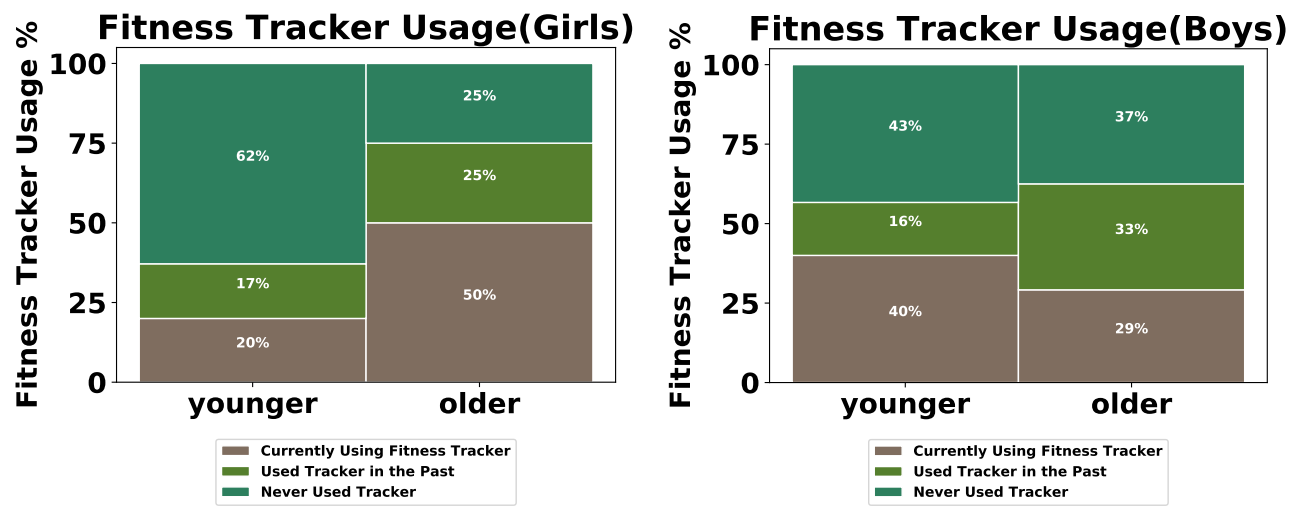

Figure 3.5: Fitness tracker usage in younger and older girls (left) and boys (right).

\subsubsection{Reasons for adopting (or not) fitness trackers}

The social influence of family and friends has been shown to have a positive effect in motivating both children and adults towards physical activity [57]. I found similar results, as all but one child who currently uses a fitness tracker have parents that use one too. This could correlate to another observation made from parents' responses when asked "Why did they (the parent) consider getting a fitness tracker for their child" where $50 \%$ (19 of 38) of parents of $\mathrm{T}_{\text {current }}$ reported 
that it was because their child asked for one (see Table 3.2).

Table 3.2: Parents' response related to why they bought a fitness tracker for their child

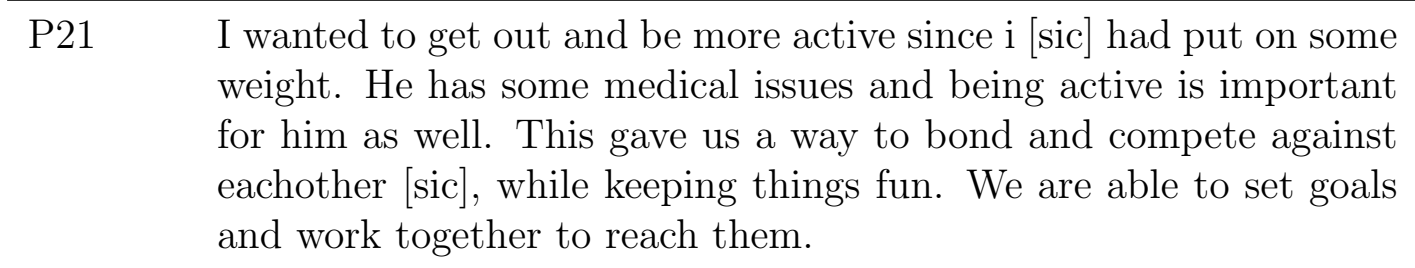

P34 Several of her friends have them, and she wanted one too. I figured it was good so she can be aware of how active she is on a daily basis.

P41 He wanted to use one of our old cell phones, and we use fitness trackers on our phones during our walks so he wanted one for himself.

Getting a fitness tracker for their child, also depends on the parent's perception of whether they think their child is active enough or not. Table 3.3 and Table 3.5 list some of the responses received for adopting (or not) the technology. Based on their personal perception parents were asked whether a fitness tracker helps/helped their child be more active. A numeric value was assigned to all possible options: a score of +2 was assigned if they indicated 'Strongly Agree', +1 if 'Somewhat Agree', 0 for 'Neither Agree nor Disagree'. In a similar manner -2 was assigned if the parents indicated 'Strongly Disagree', and -1 if they 'Somewhat Disagree'. It was observed that, parents whose children currently use a fitness tracker tend towards agreeing on the fact that fitness trackers make their children more active (see Fig 3.6, $\mathrm{T}_{\text {current }}$ ). On the other hand, for children who used a fitness tracker in the past and are not currently using one, parents had a perception leaning towards the fact that they do not agree that fitness trackers helped their child to be more active (see Fig 3.6, $\left.\mathrm{T}_{\text {past }}\right)$. This could have been one reason why parents did not consider replacing the 


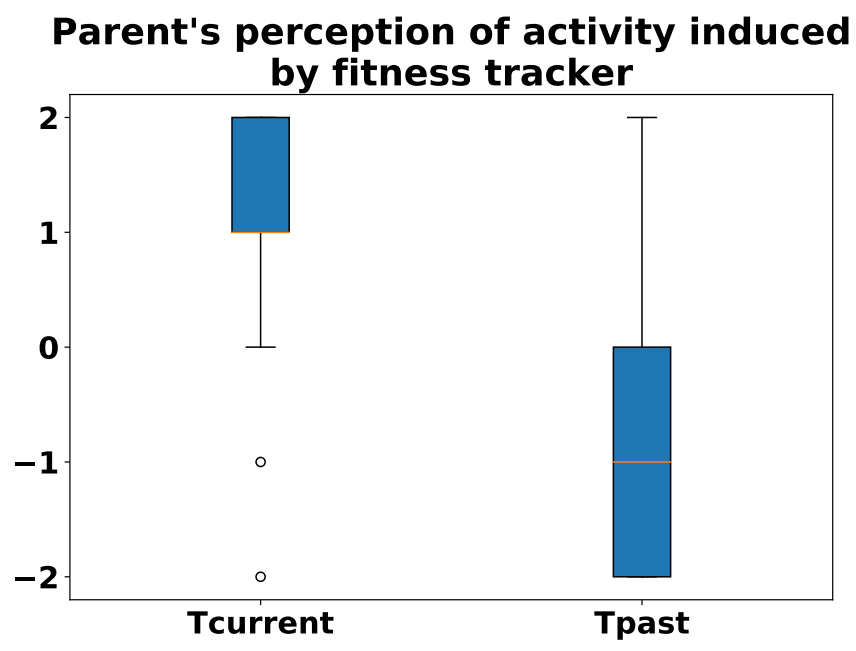

Figure 3.6: Parents' perception related to activity induced by fitness trackers in children $T_{\text {current }}$ and $T_{\text {past }}$.

fitness trackers for children who used one in the past. Another observation that shows that fitness tracker usage in children depends largely on parents' perception is that although $45 \%$ of the children in this survey did not fall in a healthy BMI range (5th to 85 th percentile), less than $10 \%$ parents rated their children as sedentary.

Table 3.3 lists some of the parent's responses with regards to why they would not buy a fitness tracker. These comments show that some parents only consider the need of fitness trackers, if they believe their child is underactive. They do not perceive fitness trackers as a technology that helps their child stay aware of his/her activity levels. Other reasons mentioned included: a concern for children's privacy and security; and, the desire for being able to encourage a natural habit not facilitated by technology.

Of the children who currently use a fitness tracker $\left(\mathrm{T}_{\text {current }}\right), 63 \%(24$ of 38) utilized a FitBit. At the time of the study, Fitbit had not yet released the FitBit Ace, its fitness tracker for children, so most children were using trackers 
designed for adults. While there are several fitness trackers available in the market designed especially for children, parental purchasing choice or social influence may have led the children to primarily use that brand.

\subsubsection{How children use fitness trackers}

Although the prescribed activity guidelines for children suggests 60 minutes of activity daily, most parents responded to the question of how their children use a fitness tracker in terms of steps accumulated or distance covered. Out of 38 responses to the question "How does your child use a fitness tracker?", 45\% (17 of 38) were generic statements about wearing them on the wrist, $55 \%$ (21 of 38) mentioned something more specific that included goals or tracking metrics. $76 \%$ (16 of 21) specifically mentioned that their children used fitness trackers for tracking steps or distance and achieving goals. None of the responses mentioned tracking activity time, although 19\% (4 of 21) responses mentioned tracking activity in general and one (5\%) parent mentioned that their child used it "in game to earn virtual items" (no game was specified).

Since most fitness trackers and pedometers are designed for adults, their primary display is in the form of steps. This could be one reason that children and parents were tracking steps or distance instead of activity time. Another reason which could have been a driving factor for children counting steps is that over $60 \%$ of children who were using a fitness tracker $\left(\mathrm{T}_{\text {current }}\right)$ reported using FitBit, which primarily displays user activity in terms of steps taken or distance covered. When asked how children use a tracker most of the parents mentioned that they use it to track steps or distance during physical activities (see Table 3.4). 
Some of the children used cellphones with built-in step counters or fitness mobile applications. Six parents mentioned that their children only wear activity trackers while doing physical activity. One parent mentioned that they their child was not allowed to wear a tracker during organized sport activities like football where it could result in an injury (a concern previously raised in [56]).

From the activities reported by parents, it was observed that children who use a fitness tracker have the highest mean number of average active minutes per day calculated over a period of 7 days ( $\mathrm{T}_{\text {current }}: \mu=92$ minutes), followed by children who used a tracker in the past ( $\mathrm{T}_{\text {past }}: \mu=79$ minutes), followed by children who never used a tracker ( $\mathrm{T}_{\text {never }}: \mu=67$ minutes) (see Fig 3.7 ). While not statistically significant, this perhaps suggests that fitness trackers may have helped children be more active through awareness which is consistent with previous studies [55]. Another observation is that all parents perceived the sum of their child's activity averaged over the week to be above the 60 minute guideline each day $(100 \%, 113$ of 113), although some of the parents still mentioned that their child needed a tracker because they are underactive or otherwise unhealthy (9\%,6 of 66) (see Table 3.5).

While most parents reported that children used a fitness tracker for tracking steps and achieving goals, it was parents who took care of charging the device in most cases. In fact out of the 38 children who currently use a tracker, 25 parents took care of charging or helped their children in doing so. However, both parents and children looked at the results which were later used for setting goals, tracking them, personal challenges, rewards, and competitions.

Some parents mentioned PokemonGo or games that let them be active with their favorite TV characters as something that could help motivate children to be active. Games and other technology based on incentives and rewards were also mentioned as 


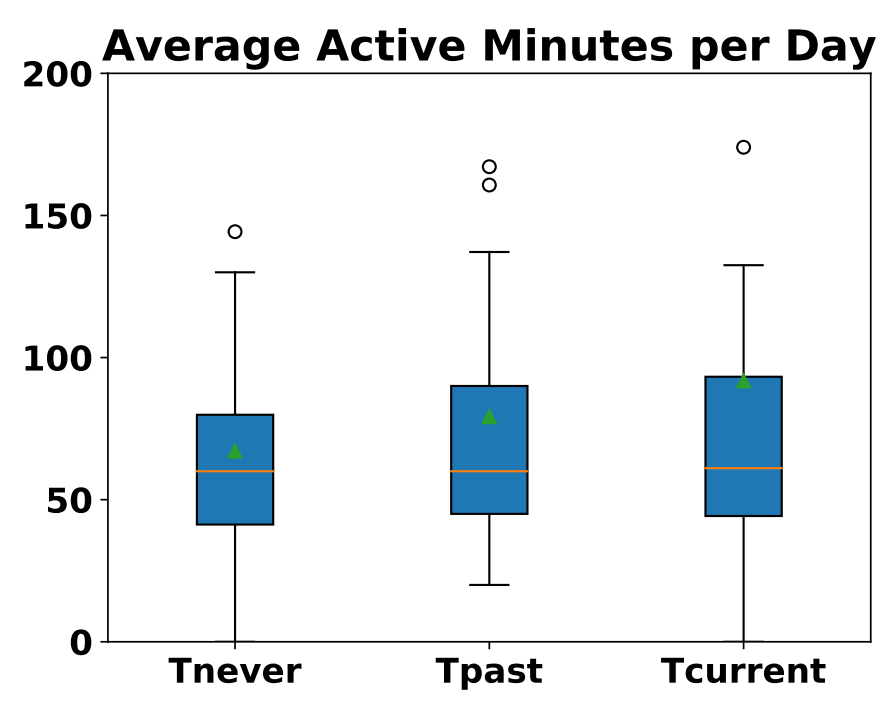

Figure 3.7: Average number of active minutes per day, as calculated by activities (e.g. walking, cycling), the number of days each activity was performed, and the daily duration of each activity as reported by parents in the last 7 days.

other alternatives to fitness trackers.

\subsubsection{Parents' perceptions of fitness technology}

From the qualitative responses to the survey, it was observed that children's fitness tracker usage is largely dependent on parent's perceptions. The driving factors for getting or not getting a fitness tracker include whether the parent uses an activity tracker ( $97 \% 37$ of 38$)$, the child's activity level (36\% 18 of 50$)$, age (14\% 7 of 50), social environment (14\% 7 of 50 ), technology exposure (10\% 5 of 50), and data security (4\% 2 of 50) (see Tables 3.2, 3.3, and 3.5). It was observed that several parents did not consider that a fitness tracker would help their child be more active nor help them be aware of their activity levels - particularly if they were already perceived their child as active. They perceived fitness trackers as technology that 
could possibly help improve activity if they think their child is not active enough. Fitness trackers for children are widely available but they are not popular. In our sample, only two mentioned trackers that could have been designed for children. A reason for this could be that parents - who generally purchase technology for their children - may purchase what they are familiar with (e.g a specific brand like FitBit), and therefore the technology used by children is frequently designed for adults. This could have also contributed to a majority of parents reporting children's goals in terms of steps.

Eight parents indicated that they acquired (or may want to acquire) fitness trackers for their family as they viewed it as a healthy activity they could do together (see Table 3.6).

\subsection{Discussion}

From the primary findings of our study, I see that fitness tracker usage in children is correlated with whether or not the parent uses a fitness tracker and parents' perception of the child's activity, age appropriateness, and suitability of the technology itself (in terms of privacy protections). The majority of parents of younger children preferred not to get a fitness tracker for their child as they consider it unnecessary. Parents indicated they would consider buying a fitness tracker for their child if they thought that the child was underactive. Social influences also affected the acquisition of trackers among children, meaning children who knew someone that used trackers were more likely to want one. Some parents also mentioned that they either currently do or wanted activity to be something that the family members can do together.

Fitness trackers have the potential to motivate adults and children to be physically 
active. However, children have different needs, motivations, interaction styles, and perspectives than adults as they utilize technology. From the responses in the survey, it was observed that the most common reason for children not using a fitness tracker anymore was being bored or losing interest, as was also reported by [56]. This could be due to the fact that a lot of them were using fitness trackers designed for adults.

Fitness trackers when designed for children, should address their needs according to their age, gender, and overall development. The responses to the survey also showed a focus on steps and distance as a metric to measure daily activity instead of active minutes as is prescribed for children. While this is an interesting finding, this could be biased because that may be the focus and perspective of their parents.

In this survey, I was anticipating parents to report children's activity in terms of duration as activity guideline for children suggests 60-minutes of activity each day. I did not ask about children's activity in terms of average steps per day (since having a tracker was not required). However almost all of the parents reported children's activity and their goals in steps accumulated each day. This indicates that as children tend to follow adults, they prefer tracking activity in terms of steps rather than activity minutes. This is one of the reasons that I decided to use steps as a measure of activity in KidFit Suite. The average steps can be translated to activity minutes using [12]. 
Table 3.3: Parents' perceptions related to why children do not need a fitness tracker (besides being too young)

P13 I don't think it's necessary at this point. He is very active and at a healthy weight for his age and height.

P46 I don't think it's necessary. Kids have enough exposure to technology as is.

P60 I don't think it is necessary. I can tell from his day to day activity whether he is healthy or not.

P90 I think it would be too much of a distraction for him. Plus I'd worry about him losing or breaking it.

P27 Don't want a child monitored by technology that could have data sold/used

P31 I don,t [sic] think it would be accurate. My son is highly active and also he would lose or break one.

P89 They are just outside playing like any other child. I really do not see the need to "monitor" how much of a workout they are getting while playing with friends or going to the park.

P98 If he was interested in one, I'd get him one, but he indicated that he wouldn't see much use for it, so I do not plan on wasting money on one at this time.

P100 I think a fitness tracker would be a great way to monitor her physical activity because I have to push her to keep her active. Unfortunately, right now she doesn't have a smart phone, and we have never really looked into getting a separate fitness tracker for her.

P105 I think creating a natural habit is better than a technology induced habit

P109 I don't see the point in it. I don't see there being a certain number of steps or anything that will deice [sic] if she's healthy or unhealthy. I can keep pretty good track of her activity levels. If I see her being lazy and I'm home I'll do something with her, whether that's walking the dog, playing ball outside or whatever else. I would rather that she not be so reliant on technology at such a young age. 
Table 3.4: Parents' response related to how their children use fitness trackers

\begin{tabular}{ll}
\hline P97 & $\begin{array}{l}\text { She likes to see how many steps she's taken total and setting a goal } \\
\text { for steps to take. }\end{array}$ \\
\hline P11 & He uses it to track steps he takes while playing soccer. \\
\hline P22 & They count their steps on various activities for mostly fun \\
\hline P34 & She uses it to count her steps per day \\
\hline
\end{tabular}

Table 3.5: Parents' responses related to why would they buy a fitness tracker

P21 I wanted to get out and be more active since i [sic] had put on some weight. He has some medical issues and being active is important for him as well. This gave is a way to bond and compete against eachother [sic], while keeping things fun. We are able to set goals and work together to reach them.

P100 I think a fitness tracker would be a great way to monitor her physical activity because I have to push her to keep her active. Unfortunately, right now she doesn't have a smart phone, and we have never really looked into getting a separate fitness tracker for her. 
Table 3.6: Parents' responses related to why they acquired (or would want to acquire) a fitness tracker in order to bond with family

P51 Well, it's just built in the app, so it was free. We all need to be more active (me, hubby and daughter...) So this seemed like a good way to challenge ourselves and make it into a fun competition.

P52 When the phone is being moved it just tracks the step. So when she is walking she takes it with her. The whole family has it too. And we count the steps we took that session.

P41 He wanted to use one of our old cell phones, and we use fitness trackers on our phones during our walks so he wanted one for himself.

P87 She liked my fitbit that I got for my birthday last year, so we got her one for Christmas and we have a little competition to see who walks to the most each day. She also likes to see how much exercise she does in a day.

P111 She saw me using one and since she likes joining me for my exercise, she requested that I buy for her.

P113 I have been using one for a couple of years. He has always been interested in it. I got one for him and his older brother as a gift. They both use it daily. They like to participate in challenges and daily goals. 


\section{CHAPTER 4}

\section{WHAT MAKES PHYSICAL ACTIVITY FUN}

An important difference between fitness games and fitness trackers is the way they appeal to their audience. While fitness trackers emphasize awareness and the importance of physical activity, fitness games include physical activity as a by-product of playing a game. In the case of young children who do not necessarily understand the concepts of calories and exercise, fitness games can be really helpful in getting them active without them noticing the fact that they have been exercising. Modern fitness games include games like the Wii and Kinect/Xbox that challenge players to get physically active. Location-based games such as PokemonGo and Geocaching have also drawn attention as a way to promote physical activity [14].

As part of our research team, we work with and participate in an intergenerational design team (Kidsteam) which comprises of adults and 8 children (ages 6-11). Kidsteam works as a participatory design group where adults and children come together as a team to design and develop technology for children using the Cooperative Inquiry method [24]. The team meets twice a week during the school year for 90 minute sessions. In this chapter, I present observations from our Kidsteam where 7 children between ages 6-11 played three fitness games, namely, Zombies Run!, Virtual Walk, and NFL Play 60. The games were played over 5 sessions of 25 minutes each (on different days). Using children's responses and ratings I present the understanding 
of the key contributing factors that make physical activity fun for children and the effectiveness of these games in motivating children to be physically activity.

\subsection{Method: Participatory Design}

In order to understand the answer to the question " what makes physical activity fun for children?" I conducted five sessions with children utilizing and evaluating various activity applications: two sessions of Zombies Run, two sessions of Virtual Walk, and one session of NFL Play 60. Each application was actively used for 25 minutes by seven children in an indoor environment. They rated their experience using the app every five minutes. This was done via a simple Smileyometer ( 5 point child-friendly Likert-style) evaluation where 1 meant awful and a rating of 5 meant brilliant [63]. I present a description of each app and children's experience while they used these applications. The order of play with each application was random.

\subsubsection{Zombies Run!}

Zombies Run is a mobile, narrative-based fitness game (Fig 4.1) where players try to survive a zombie apocalypse through a series of missions during in which they run and collect items that help them survive. The narrative was played by means of an audio which the children listened to using headphones (Fig 4.2). The audio played approaching footsteps if a zombie was trying to catch the player, in which case the players is supposed to start running to escape the zombies.

The children were very excited about playing a mobile app-based game and the narrative of being chased by zombies. The joy of trying a new technology may have partially contributed to the excitement. However, they were soon bored as they 

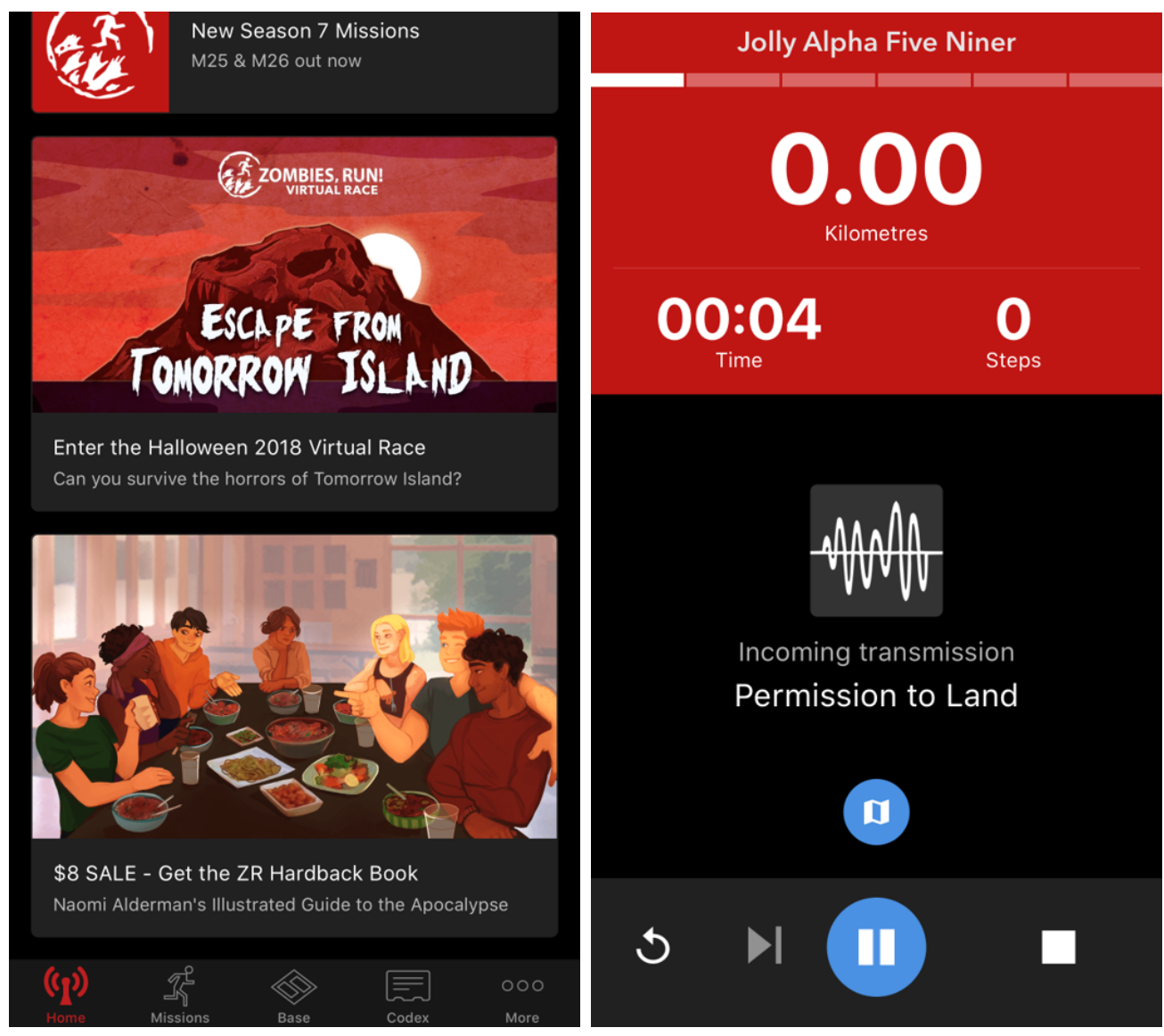

\section{Figure 4.1: Zombies Run! application.}

mentioned they wanted to be chased more often. One of the children suggested that it would be better if they could control the frequency of being chased by zombies. The children loved the audio and narrative. One said as they heard the zombies approaching: It feels like we can actually see the zombies. One child mentioned they thought the theme of zombies was perhaps too violent for children. There were also some words in the audio that were considered bad by children. As they were playing the game, they wanted to see how their friends were doing or where they were on the map of the city in the application. They suggested that it would be better if they could see their friends on the map in the app and approach them for 


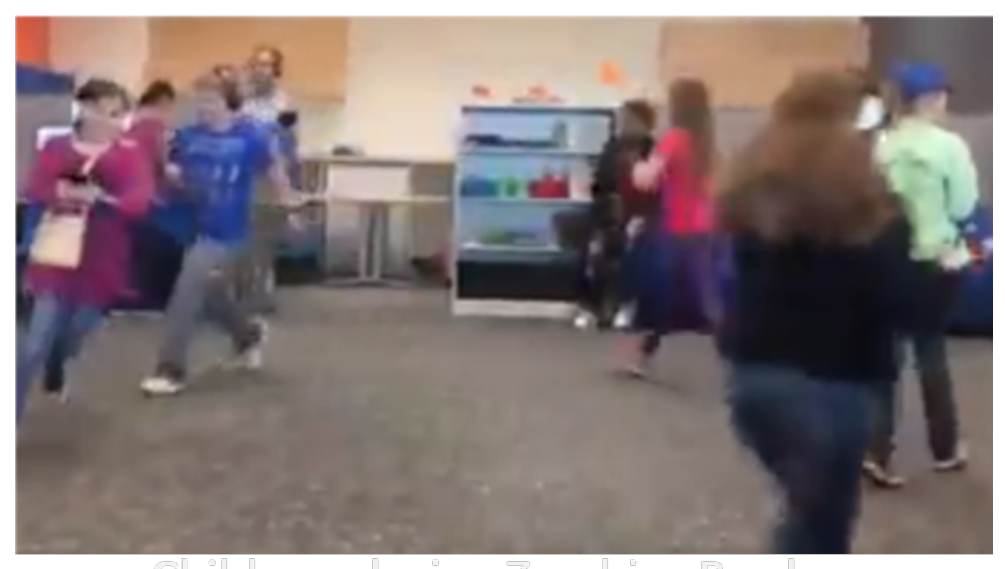

Figure 4.2: Children playing Zombies Run!

help when being chased by zombies. Their suggestion also indicated that the children preferred playing in a group more than playing on their own. Such a social aspect was missing in Zombies Run. Listening to the audio via headphones (Fig 4.2) further discouraged any communication between the children. When the game evaluation was over, the children said that they enjoyed the game but felt exhausted. Despite that, they wanted to go outside for a walk afterwards to relax, suggesting that they viewed the activity as more of a game, separate and different from their perception of physical activity or exercise. This gaming aspect seemed to have a positive impact in motivating children towards physical activity.

\subsubsection{Virtual Walk}

Virtual Walk is a mobile app that lets walkers track the distance walked instead of steps as in most fitness trackers (Fig 4.3). It does not actually track the distance either, but users define their speed through a setting in the app and the scene changes as if they were walking at that speed. It motivates activity by enabling 


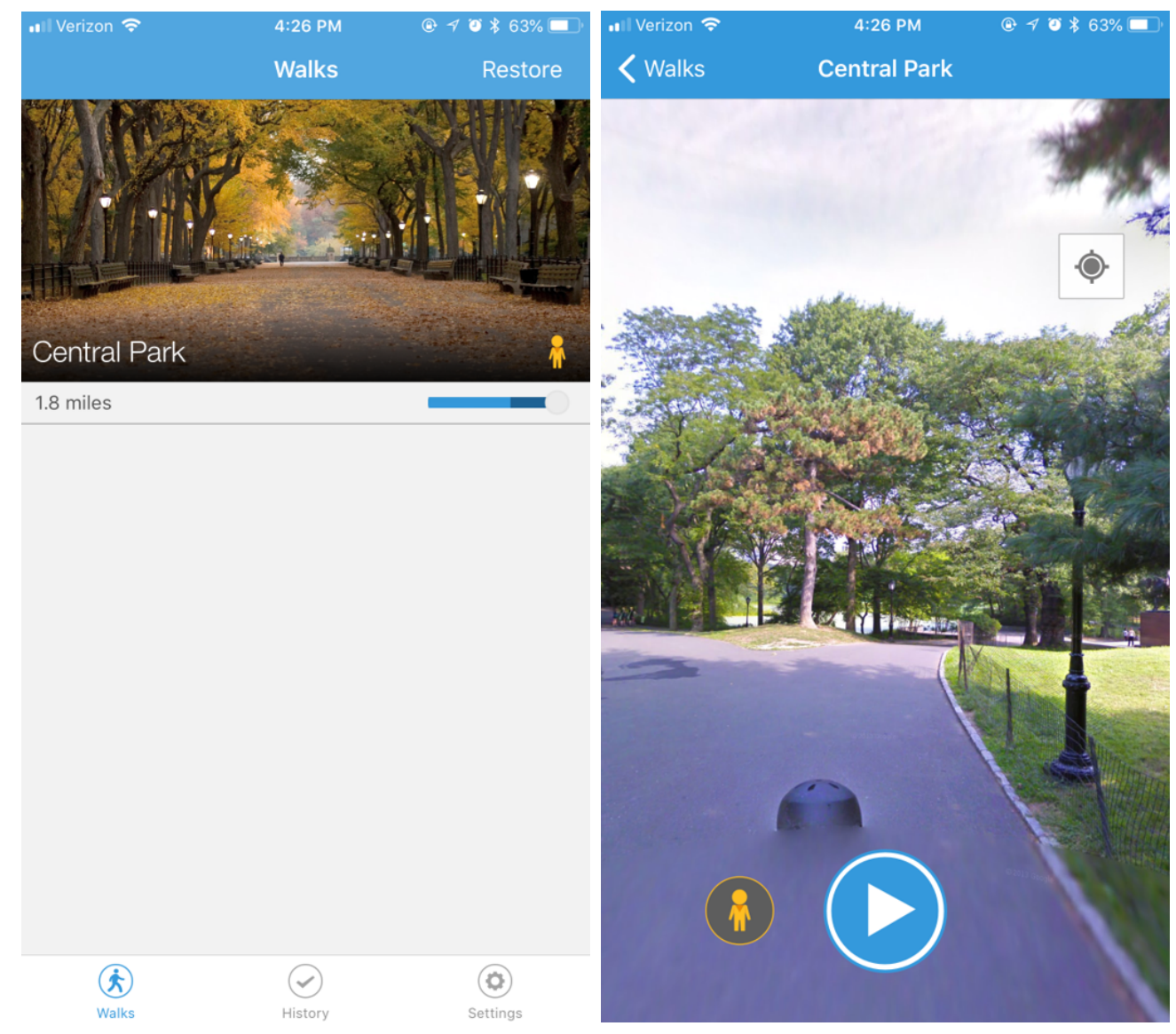

\section{Figure 4.3: Virtual Walk! application.}

a virtual experience of walking through different locations (like Central Park, the Grand Canyon, and Mt. Fiji).

In the first couple of minutes the children expected a narrative similar to Zombies Run, but when they saw that this game did not involve a narrative or rewards, they spontaneously added their own creative elements to the experience (Fig 4.4). This included running and chasing each other, walking side by side with friends to achieve milestones together, and when someone was tired they would call for a taxi and some other person would come and hold them and would walk along with them holding on like they were getting a taxi ride. Since the app did not involve any audio or 


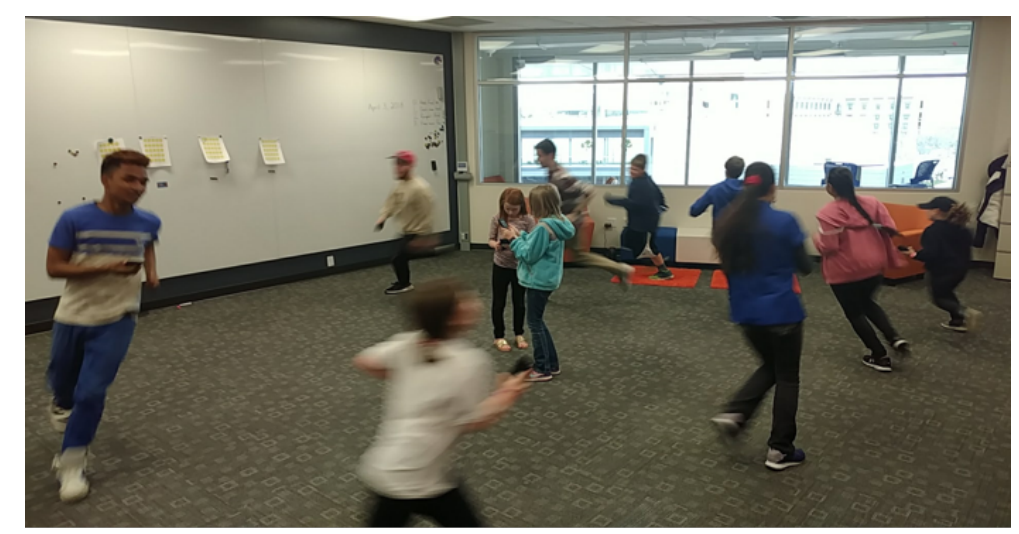

\section{Figure 4.4: Children playing Virual Walk}

headphones they could more readily interact with one another. For example, they occasionally compared their location on the map with their friends location and the other one would wait at that location as it showed on their screen, so their friends could come and join them at that location. It was here that they figured out that the app was not actually tracking steps/distance and even if they did not move while waiting for their friend the video would still continue based on the speed set on the app. This led one of them to figure out how to increase the speed and reach the destination without walking any further. Despite the fact that the app was not sensing their steps/distance, they still kept running and chasing each other (even the child who completed the walk by increasing the speed) and were involved in physical activity in a playful manner (Fig 4.4 ). In the end, they suggested that it would be better if the app actually tracked steps. As there was no audio, I noticed more interaction among children, which they really liked: one child labeled their experience as "Brilliantnes" on the evaluation sheet. 


\subsubsection{NFL PLAY 60}
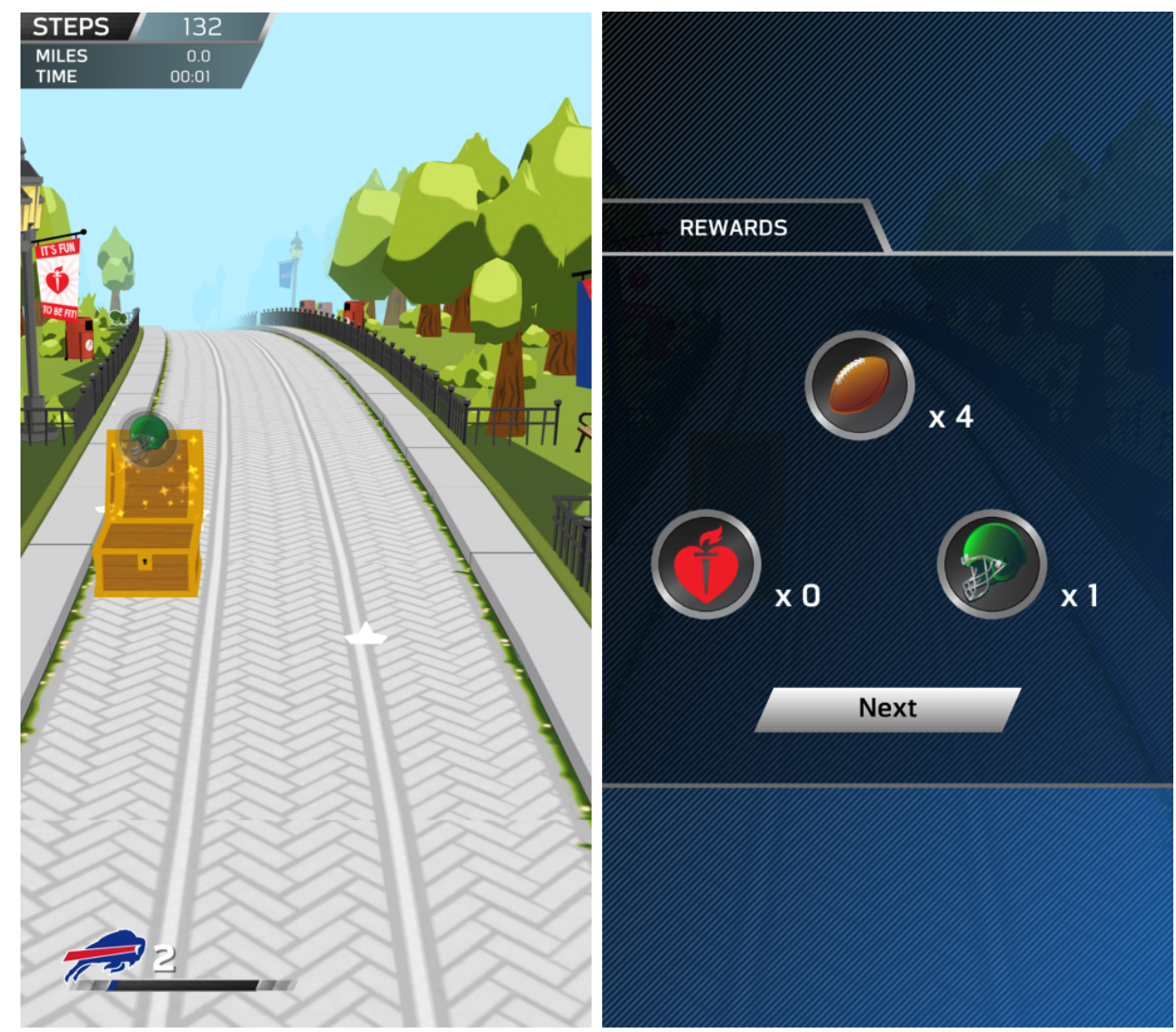

Figure 4.5: NFL Play 60 application.

NFL Play 60 gets children active through episodes of sixty minutes each to meet

the guidelines to fight obesity [8]. Within each episode, children walk their character, collect characters to build a team, train with them and collect rewards (Fig 4.5).

Unlike the other two games this game involved incentives and in-game rewards to motivate children to be physically active. Children used headphones to listen to the musical audio in the game. However, since this game lacked a narrative, children found it less appealing than the other two games: one of them commented that it 


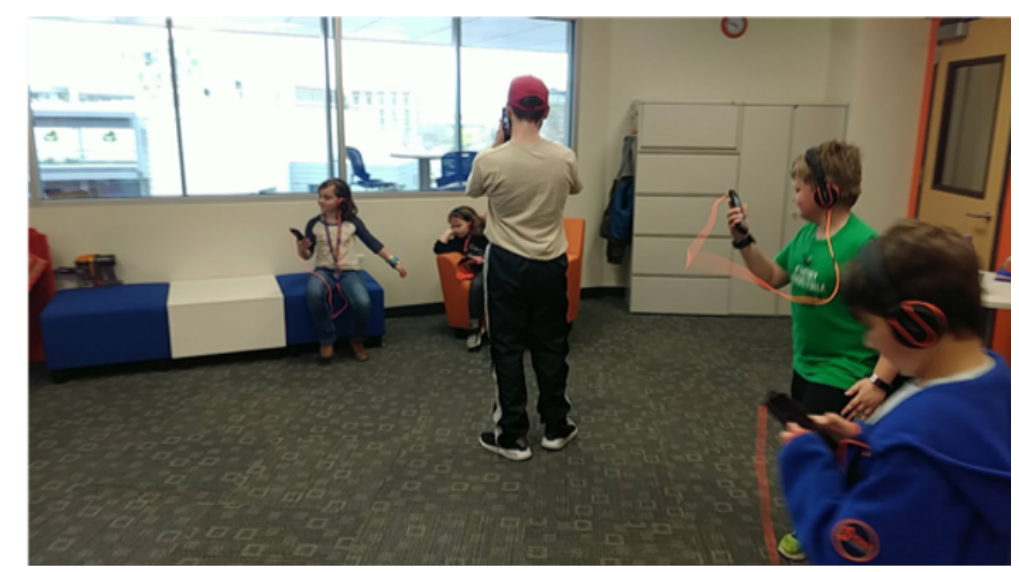

Figure 4.6: Children playing NFL Play 60

was repetitive just walking and collecting rewards over and over again and one other described it as boring. The use of headphones led to fewer interactions amongst the children and restricted the running and chasing as observed when they used Virtual Walk. As was suggested in Zombies Run, they wished they could see their friends in the game. Since the game is based on a motion sensor to track steps, one of them figured out that the step count would increase if they merely shook the phone while they were sitting down. That child did not walk after that but instead kept shaking her phone to maintain pace with the other children. Contrary to this, the game was highly rated by that child. This may be attributed to the fact that she perceived the game as an ordinary mobile gaming application. In fact, they were so bored playing the game the first time, that they refused to conduct a second session with this app. Children were not very active while playing this game compared to the other two games (Fig 4.6). This game was liked the least by all children as it neither included a narrative nor any social element. 


\subsection{Key Findings}

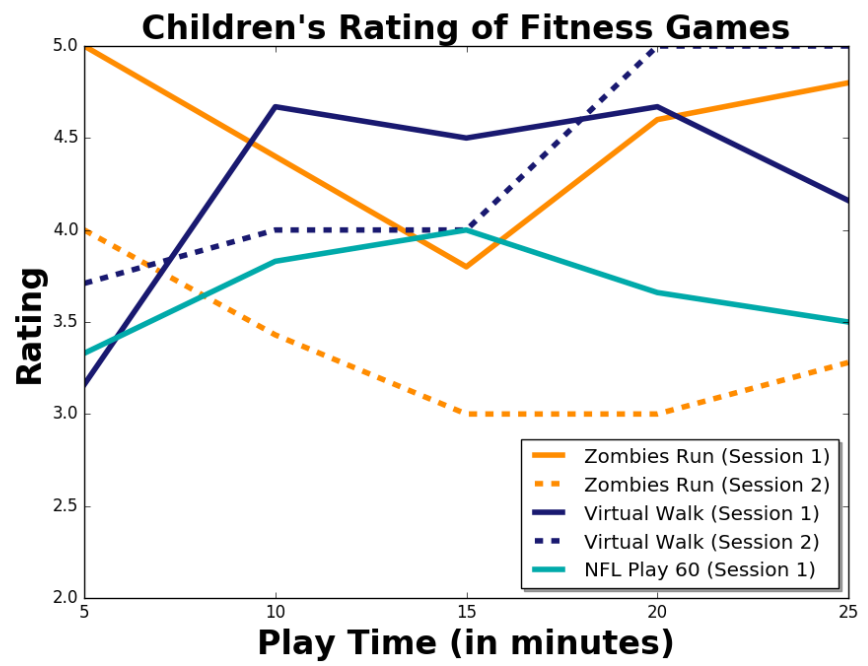

Figure 4.7: Children's ratings of Zombies Run!, Virtual Walk and NFL Play 60

The graph in Figure 4.7 shows children's ratings collected every 5 minutes using the smileyometer rating technique [63] in the 5 sessions of the games played. As can be seen from the graph, Zombies Run was not liked as much the second time as it was the first time. The narrative was found to be an important element to get children's attention and motivate them to engage with activity games. However lower ratings towards the end indicate that children enjoyed having a game with a narrative but the narrative itself does not ensure engagement into the play over time.

On the contrary, both the sessions with Virtual Walk started with mediocre ratings but were rated high towards the end by children (Fig 4.7 ). As there was no audio or headphones involved in virtual walk, it provided a way for children to interact and communicate and play together. From our observations watching them play, I can say that children preferred having a social element in the game. Because of 
the openness of choosing the type of activity or how to play provided an additional benefit. Children liked that they had the flexibility to add their own element of running together and chasing each other.

The scripted play of NFL play 60 was not liked very much and therefore was played only once. The lack of a narrative and social interaction seemed to have contributed to the lower ratings of the game (Fig 4.7). The children were so bored playing the game the first time, that they denied to play NFL Play 60 for the second time and hence only 1 session with NFL Play 60.

\subsection{Discussion}

Based on the ratings provided by the children of the three games and our observations of them playing these games, we conclude that children prefer playing physical games with: (1) social interaction, (2) narrative, and (3) the flexibility to add their own creative element. This is also supported by literature $[1,38,44,47]$. While previous researches have emphasized on the effectiveness of these elements, KidFit Suite tries to incorporate these elements under one hood. It uses and combines the findings from these researches to make physical activity enjoyable for children. The element of social interaction liked by children in these games is incorporated in the form of collaborative and competitive games. The growing of a garden of flowers or collecting jewels on a crown add the element of narration. Since the applications in KidFit Suite only track user's activity in terms of number of steps or active minutes, children can choose the type of activity and play. 


\section{CHAPTER 5}

\section{DEVELOPING FOR SOCIAL AND TEMPORAL IMPACT}

In my previous research, I found that children while interacting with mobile gaming applications enjoy social interaction, flexibility and narratives (Chapter 4) which informed the design of KidFit Suite. I utilized the elements found and combined them together in a collection of applications, KidFit Suite. This chapter presents: the details of implementation of each of the applications in KidFit Suite, the key elements employed, the interaction with the Fitbit server, the database that stores the user data and the API's that facilitate the communication between the front-end application, the Fitbit server and the database. I developed the mobile applications using Ionic framework, which is a cross-platform library that allows a single codebase to be deployed across multiple platforms. Finally, this chapter will conclude by detailing the walkthrough of the games by visualizing each page on the applications and how the user proceeds through the games. The mobile applications were developed using Ionic platform. KidFit server API's are written in PHP and use HTTPS secure communication. The database is operated via MySQL workbench which is a tool to create and manage RDBMS. 


\subsection{Key Elements in the KidFit Suite}

KidFit Suite is a collection of mobile applications developed as part of this research and employs elements of social interaction, temporal flexibility and visual persuasion to engage users into physical activity. The gaming applications lets users visualize a garden blooming with flowers or a crown being studded with jewels as they achieve in-game activity. To simplify the terminology a game with the applications refers to each session of game play. The social and temporal elements lets children choose the players in the game and the duration of the game. In this section, I elaborate on the key features implemented while developing KidFit Suite.

\subsubsection{Social Interaction}

In my previous research (see Chapter 4), I found that social interaction is an important element in engaging children in physical activity games. Children enjoyed playing the game most when they could connect and interact with their friends. This also provided children with sufficient flexibility to add their own elements of running together and chasing each other. Another similar research [32] suggests that socially educational games could benefit children. Games with a social element were found to increase social competence in shy children and promote communication and acceptance by their peers [32].

The mobile applications in KidFit Suite were developed using the method of Cooperative Inquiry [24] and participatory design principles [29, 30] where children from Kidsteam (See Chapter 4 Section 4) and adults come together as a team to design and develop technology for children. The social aspect in the applications lets children interact and play games with their peers. While playing the games children 
do not have to be co-located.

The social interaction element in KidFit Suite is comprised of two dimensions: Collaborative and Competitive. In competitive play, children challenge and compete against each other. At the end of the game, the child (or children) with maximum activity win the game. As they proceed through the game, they can see the in-game activity of their peers. Each individual has their own garden or crown and progress can be seen in terms of the number of steps accumulated or the number of flowers or gems collected (see Figure 5.11). On the other hand in collaborative type of play children are given a target to achieve in a given amount of time. Since children play as a team, only one garden or crown shows up on the screen which reflects the cumulative progress of all the participants (see Figure 5.12).

\subsubsection{Temporal Flexibility}

Children are advised to get 60-minutes of activity each day [34]. Several applications like BunnyBolt [38] and FitQuestLite [1] are developed to help children meet their daily goal of 60-minutes of activity via gaming and play. Most of these applications however, emphasize that it is easier and more practical [38] for the audience to achieve this target in short bursts rather than aiming for the entire duration at one go. I found similar results in my research where the long and scripted play of NFL Play 60 was least liked by children (see Chapter 4). On the contrary fitness applications which had 20-40 minutes of activity sessions were enjoyed better (see Chapter 4). Another aspect of temporal flexibility builds from the knowledge that every child has a different schedule. Our goal while working on these applications was to provide children with the flexibility to engage with these games when they are available opposed to something with a mandatory timeframe. 
The temporal flexibility element in KidFit Suite lets children engage with the applications according to their availability and aims at achieving the recommended activity in short intervals of play. The gaming applications in KidFit Suite let the users enjoy temporal flexibility via two types of play which are Synchronous (short) and Asynchronous (long) play. Synchronous play was designed to last for a short period of time between 25-45 minutes and expects all the users to play at the same time. In a synchronous collaborative game, children needed a total step count of 2000 times the number of players in order to win the game. Asynchronous play on the other hand lasts for a few hours and does not expect participants to be active for the entire duration. Children have the flexibility to play intermittently when they are available. They can choose to play for some time, get some rest and play again when they want to. The duration of play for an asynchronous game was decided such that if the number of players in a game were more than 5 , the duration of the game would be a random number between 25-45 minutes times the number of players. When the number of players is less than or equal to 5 , the duration of the game is 1 hour plus 25-45 minutes times the number of players. This ensures that the duration of the game is at least 2.5 hours but not more than 6 hours.

\subsubsection{Persuasive Display}

Several researches have utilized the persuasion brought by displays. Some examples include Ubifit [22], ActivMon [18], and Fish 'n' Steps [44]. These applications build upon the fact that seeing one's progress through a visual display is more appealing than merely watching the numbers. In fact for young children, understanding the value of the numbers could be complex. For instance some children in our Kidsteam design sessions got confused about 1000 and 10000 . Creating activity awareness in 
children through a visual display makes understanding and interpreting their activity levels easier. This also lets them easily compare their activity with the activity of their peers.

KidFit Suite lets children visualize and compare their in-game physical activity through a simple and easy to understand display. Capture the Crown displays in-game user activity via means of jewels showing up on a crown. Grow the Garden displays in-game user activity via a blooming garden.

In the game Capture the Crown the user's progress in the game or the in-game activity is notified to the user both by numbers and by means of jewels on the crown When the game starts based on the type of play (collaborative or competitive) there are one or multiple crowns or gardens on the screen. Since in a collaborative play all children work towards a common goal, only one crown or garden representing the group's total activity is displayed. While, in competitive play every child competes against others therefore each player has their own crown or garden representing their activity. As the game proceeds based on the activity of players jewels start showing up on the crown or flowers start showing up in the garden up to a maximum of ten. In collaborative play, if the children are able to collect all 10 gems or flowers as a team, they win the game. In a competitive game the child with maximum steps and therefore jewels on the crown or flowers in the garden wins the game. The user can see the progress both in terms of steps achieved and flowers/jewels on their screen.

KidFit Suite contains the two themes of flowers and gems but other themes could be used as well. In one of our design sessions with Kidsteam, we played a game with another theme, named Escape the Tunnel where children's activity helped them escape a tunnel. As they progressed in the game, their screens got brighter to visualize actually escaping a tunnel. Children (Kidsteam) liked the concept of visual display 
and they actually felt like they were escaping a tunnel (apart from the brightness of the screen, I also modulated the lights in the room to create the effect).

\subsubsection{Flexibility of Play}

In our previous research (see Chapter 4), I found that while interacting with fitness games, children prefer to choose the type of activity. They enjoy adding elements of running and chasing and the freedom to play as they like. This flexibility of play, where children can choose the type of activity, is one important element in KidFit Suite. KidFit Suite does not tell a child to run or walk or do jumping jacks like in some exergames, instead it lets them choose the type of activity.

\subsubsection{Steps as a Measure of Activity}

The World Health Organization recommends at least 60-minutes of activity each day for children and youth in the age group 5-17 years of age [11]. These active minutes should be accumulated via moderate to vigorous activity such as sports, physical education, exercise, chores etc. While there are plenty of fitness trackers available, most of them report activity in terms of steps accumulated and/or distance covered, there are a few like Fitbit that also measure active minutes. These active minutes start accumulating only when the user is 40 percent above their resting heart rate

for 10 minutes or more [3]. In simple words, on a Fitbit (used for measuring activity in this study) it shows no change in active minutes until you have a difference of at least 10 active minutes. This was one challenge faced while designing the apps with Kidsteam. The applications in KidFit Suite facilitate the flexibility of achieving the daily activity guideline via short intervals of play. In the current implementation using steps as a measure of activity tracking, during the short periods of play children 
see a flower or a gem every 250-500 steps. However, it was not possible to show a flower or gem every 2-3 active minutes as Fitbit does not report activity until there is a difference of 10 minutes or more. This also hinders with temporal flexibility to play as and when they are available. KidFit Suite uses steps as a measure of activity and it shows the user their activity both in terms of steps and active minutes. Since steps can be reflected almost instantly, it proved to be a better motivation in one of our design sessions as children could instantly see the steps achieved unlike the active minutes.

While not used, the steps achieved can be converted to relevant activity minutes based on strategy presented in [61]. It looks into how the steps can be converted into equivalent active minutes based on age and gender of the child indicating steps could be used as a measure of activity for children.

\subsubsection{Secure and Easy Login}

KidFit Suite stores the user data, their fitness data and games in a secure database. This information is sensitive particularly to children. This enables children with access to social games and secured data. When the user registers with KidFit system for the first time, they are presented with an authentication page by Fitbit where the user can choose to allow the application to access their Fitbit data. Upon successful authentication, the back-end API's returns an access token which is stored in the device along with the email address. This access token is then used to communicate with all the other KidFit API's. This also saves the user from the trouble of logging in every single time they open the app. However, the access token expires after one month if the user has not logged in and in such a case, the application will require them to log-in again using their credentials. The mobile applications, the KidFit API's or 
the database do not directly store any textual passwords. The user passwords are doubly encrypted before storing in the database, the first encryption occurs in the application so as to assure secure transmission and the second encryption occurs in the back-end API's before storing it in the database. KidFit Suite applications ensure data privacy and are designed to be secure.

\subsection{Overview of Implementation}

\subsubsection{MySQL Database}

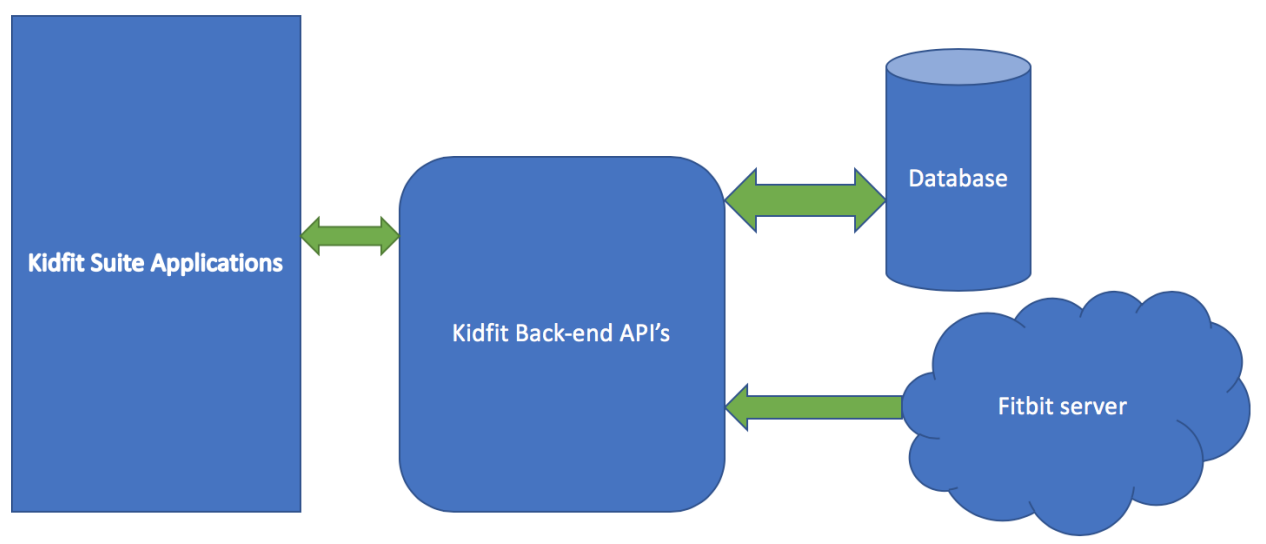

Figure 5.1: Data Flow between the application, back-end API's, Databse and Fitbit server

A database system lets developers easily store, manage, and organize data in forms of tables and relations. KidFit database stores user and game related data thereby providing users with features such as Fitbit authentication, ability to invite 
their peers for games, play with them, and see the steps they achieved in a game, and the number of games won or lost. KidFit database also facilitates the flow of data from the Fitbit server API's and the KidFit API's to the mobile applications. Fitbit limits the number of times an application can access data from the Fitbit API provided the user has authenticated the app to 150 per hour [9]. In order to present the user with the most recent fitness data and still not exceed the limit of API accesses by Fitbit, the database needed a table to store the fitness data of the user. The current implementation ensures not exceeding the limit of 150 by fetching new fitness data from Fitbit API's if the fitness data in the database is more than a minute old. A user table stores user data such as email address, age, gender, alias, access tokens, encrypted password for login in the app, Fitbit user identification and more. The mobile device registration is also saved to facilitate notifying a user using google firebase. Figure 5.2 shows the entity relationship diagram of various tables used in the KidFit database.

\subsubsection{FitBit Data Access}

Fitbit lets an application access users activity data from trackers and manually entered logs via API's provided that the user authenticated the application to do so [9]. Using this activity data from Fitbit, KidFit Suite lets children collaborate or compete with their peers to play physical activity games. This data fetch from the Fitbit server happens in the following stages.

\section{Obtaining Authorization}

While registering with the KidFit system, the app takes the user to the Fitbit authentication page. Upon successful login into the users Fitbit account, Fitbit 


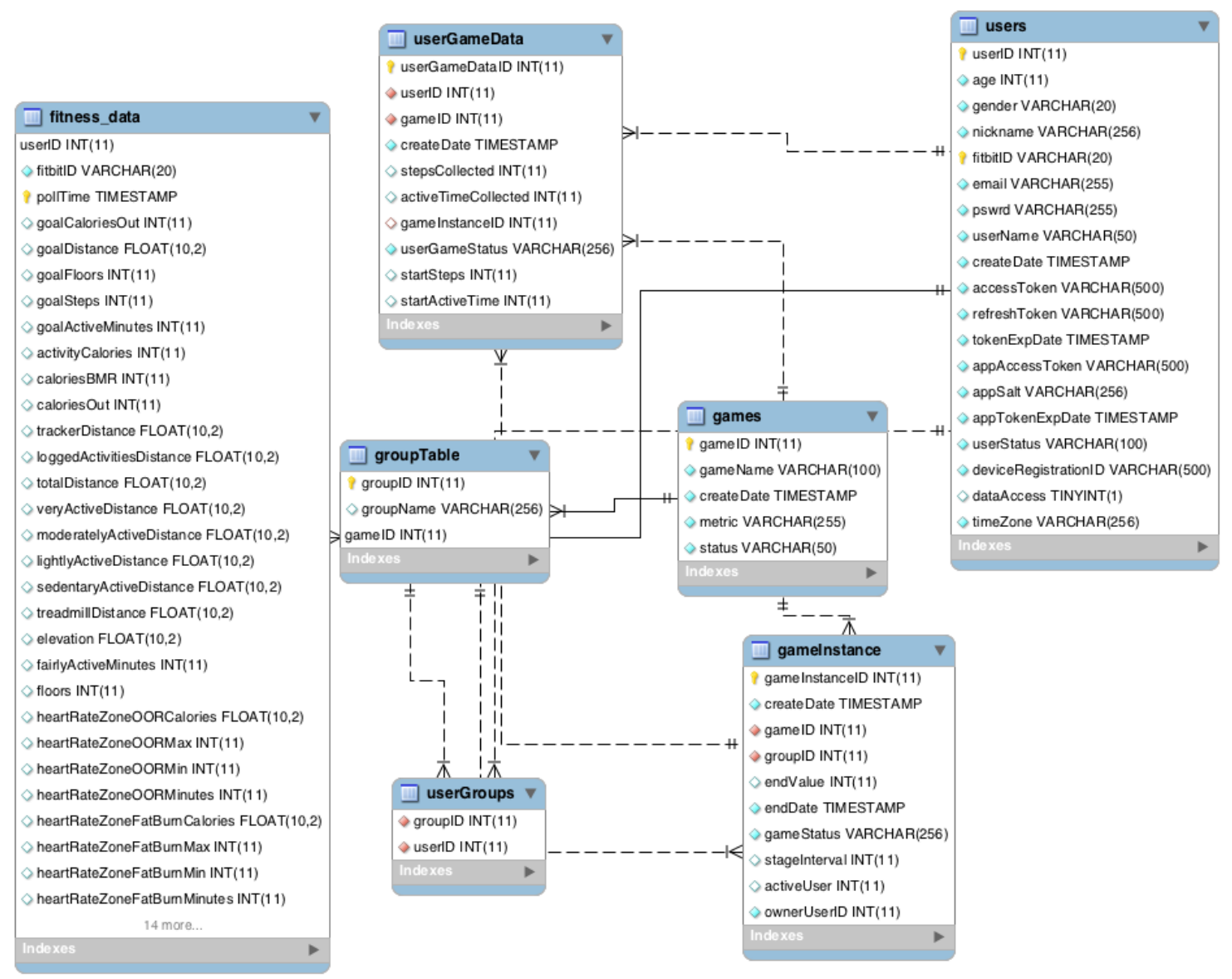

Figure 5.2: Entity Relationship diagram of KidFit Database

presents a user with a list of their fitness data that they decide to grant access to the application (Fig 5.5).

\section{Response from Fitbit server}

Upon receiving authorization from the user, Fitbit redirects the user to a redirect URL provided by the developer. The URL contains an authorization token. The page then exchanges this authorization token to fetch the user access token and refresh token and Fitbit user identification. All further data access from the Fitbit server is done 
using the user identification and access token provided by the Fitbit server. The access token is valid for a period of 8 hours by default, upon which the refresh token and the user identification are used to fetch a new access token for the user.

\section{API access and fitness data}

The application can select all or some of the user's fitness data based on the API URL and parameters passed [9]. KidFit Suite fetches the entire fitness data which contains steps, active minutes, sleep, heart rate, the timestamp of the last time the data was synced and more. The active minutes returned in the fitness data are comprised of lightly active minutes, moderately active minutes and very active minutes. Only the moderately active minutes and very active minutes count towards a user activity minutes as reported by Fitbit. The last sync time is used to make sure that every player in the game has synced their fitness data at or after the game end-time to ensure their in-game activity is correctly recorded. There are however a few limitations that were faced while fetching the Fitbit fitness data. They are:

- Syncing with the Fitbit app: The way the data access from Fitbit servers works is that the Fitbit API's return the fitness data at the time of last sync with the Fitbit app. So if the user accumulated some activity and did not sync their Fitbit app, the Fitbit server will still report the old data. As KidFit API's communicate with the Fitbit server and not the device directly, the data reported may be old and inaccurate if the user has not synced their Fitbit app recently. This causes an overhead on the user to make sure that they sync their Fitbit app in order to get their activity reflected in the gaming applications. When working in one of our design sessions with Kidsteam, a few children lost the game because they did not sync their Fitbit app in time. This led us to 
bring an end-game logic such that the last sync time of the user has to be greater than or equal to the game end time for the game to end. This ensured that their in-game activity counted towards the game. This also justifies choosing steps as an activity measure which are reflected instantaneously rather than in intervals of 10 minutes like activity minutes.

- Limitation on the number of API access within an hour: Fitbit restricts the number of times API's (fitness data and last sync time are two different accesses) can be accessed to a max of 150 within an hour per user [9]. Since children want to see their in-game steps achieved almost instantaneously, this was a limitation faced. The current implementation is such that when a user or the app tries to fetch fitness details, the database is first checked. If the entry in the database is more than a minute old, new data is fetched from the Fitbit server, saved in the database with the updated timestamp and returned to the user else the latest data from the database is returned. This makes the user wait a minute to see their activity in the app but avoids exceeding the limit of 150 provided by Fitbit.

- Intra-day Data Access: The default access to Fitbit fitness data only allows the developer to fetch the latest instance of activity data on a given date. In order to fetch the fitness data within a given period of the day, intra-day access is required. In other words by default Fitbit does not allow applications to fetch data for the duration of the game. In order to compute the exact in-game activity intra-day access is required. My request for intra-day fitness data access was sent in October 2018, and approved in November, but I'm still waiting for the access. The lack of intra-day access limited KidFit Suite to present the 
correct in-game activity achieved. As an example if the user did not sync their Fitbit data with the Fitbit app for the entire day, started a game and then synced the data, the entire data for the day would count towards the game which actually was not the the in-game activity. This was one limitation faced by KidFit Suite applications.

\subsubsection{KidFit API's}

KidFit API's facilitated interaction between the applications and database and provided data abstraction by only presenting the child with the information relevant to them. These API's let children register with our system, login, check their fitness data during the day, invite friends for a game, accept or decline game requests, start a game, see in-game activity and more. The details of KidFit API's are in Appendix A.

\subsubsection{Data Flow}

As shown in Fig 5.1, KidFit back-end API's facilitate the communication and data transfer between different components of KidFit Suite. When an application tries to $\log$ in the user, or display the games they are invited to, the application calls the relevant API which then communicates with the database to fetch the data and present it to the application. Similarly when the user wants to know their current activity status, or in-game activity so far, the KidFit API's check the fitness data in the database for that user. If the data is less than 60 seconds old, the data from the database is returned else new data is fetched from the Fitbit server, written into the database and then returned to the applications. The data flow from Fitbit server to API's is unidirectional as API's do not write any data on the Fitbit server but 
just read the current fitness data. To prevent accidental manipulation of data in the database by the user or the application, there is no direct communication between the two. The KidFit API's first verify the user and write or read the desired content to or from the database.

\subsubsection{Ionic}

Ionic is a web-based cross-platform mobile application framework which leverages AngularJS and Cordova. AngularJS is used to manage separating concerns in order to modularize things like view definition, UI hooks, and data flow. Ionic makes use of well known web technologies like NPM, HTML, CSS, JS, and Gulp to build web applications which run locally on the mobile device. Due to Ionic's popularity, Ionic has an extremely active and helpful development community. This, coupled with the feature allowing Ionic applications to be built directly in the browser (provided that there are no device API calls), means that debugging errors is by far the simplest in Ionic. Furthermore, because Ionic leverages Cordova for device API calls, Ionic is able to build executable binary files for a wide variety of platforms, including iOS, Android, Windows phone, Blackberry, and Ubuntu. This allows mobile applications to be built for a large number of devices. While Ionic is an attractive web framework, it is not without its caveats. At the time of development, Ionic was experiencing a version change from 3.X to 4.X such that some functionality implemented on 3.x code did not work the same way on 4.x. Application stability is of great importance in this work, so the more mature version 3.x was chosen. 


\subsection{Game Walkthrough}

This section elaborates on interacting with the mobile applications and creating or playing a game. The various screens and the functions are shown below:

\subsubsection{Welcome View}

The welcome view (see Fig 5.3) welcomes you to the application. If you have previously logged in the system and not logged out, as previously mentioned, the app stores the email address and access token of the user. This lets them directly login into the system. As the user hits the Get Started button, if the credentials are stored they are taken to the games page (Section 5.3.4), if not they are taken to the login page (Section 5.3.2).

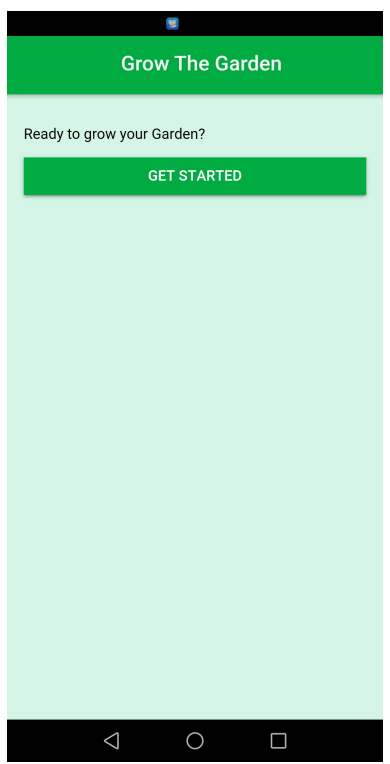

Figure 5.3: Welcome view 


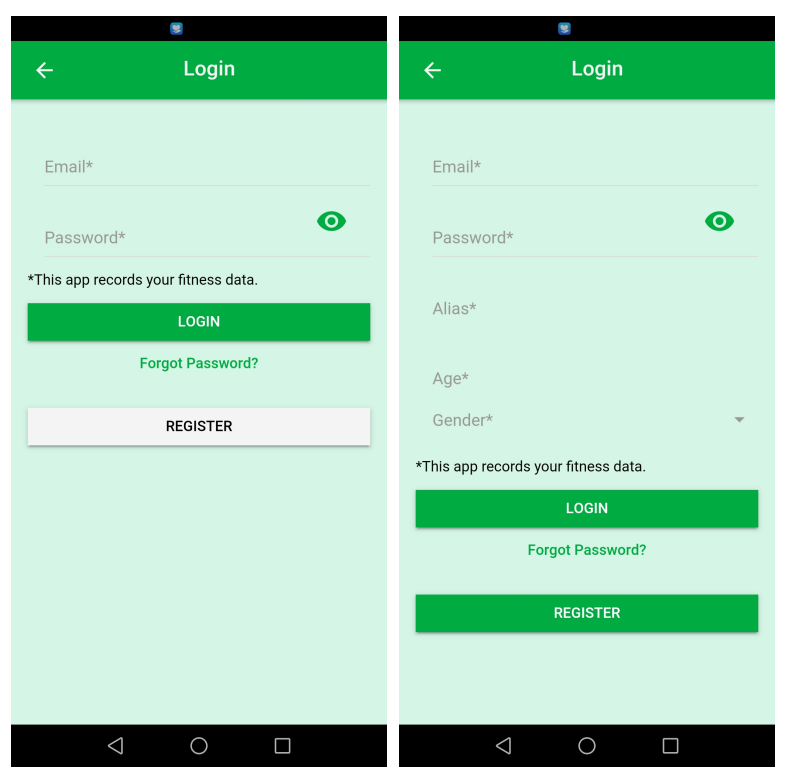

Figure 5.4: Login and Register views

\subsubsection{Login/Register View}

Fig 5.4 shows login and register view of the applications. The user needs to enter an email address and password to log-in to the system. The eye icon lets the user view or hide the password. The register button will open the register view of the app. The register operation is generally used only once while login button will be used frequently hence the register button is grey initially but turns green while registering (Fig 5.4). The user can click on the forgot password text if they forgot their password and want to reset it as is elaborated in Section 5.3.3. The register screen expects the user to create an account with KidFit applications using an email address. Other fields are password, alias, age and gender. When registering, the applications seek user authentication to access their Fitbit data and hence will be taken to a Fitbit login page (Fig 5.5) followed by authentication page. 


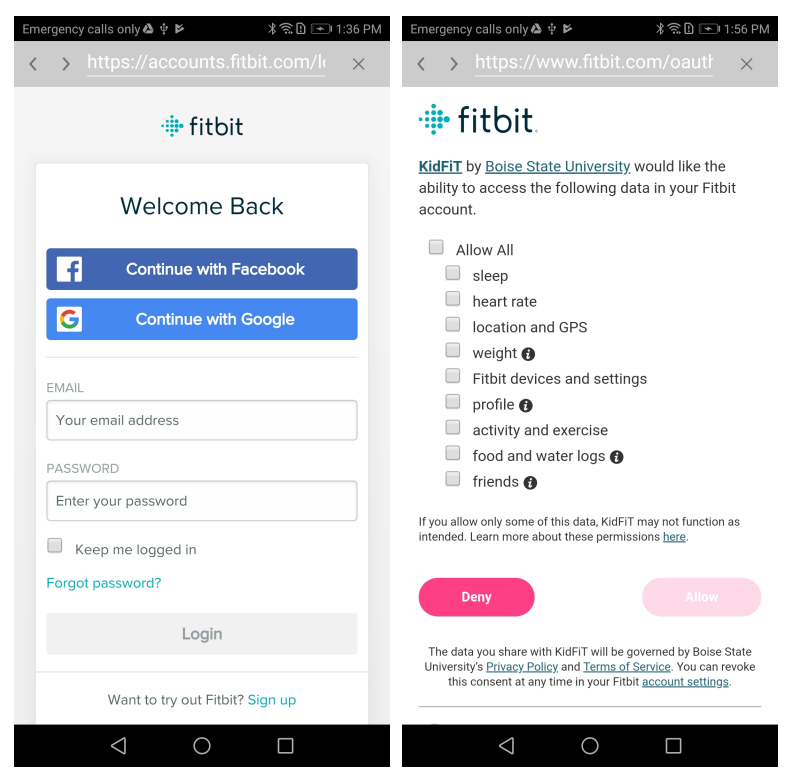

Figure 5.5: Fitbit Login and authentication views

\subsubsection{Forgot Password View}

The forgot password view lets a user reset their password if they are not able to recall it. As the user clicks the forgot password option, (see Fig 5.4) they are taken to the reset password page, where users are requested to enter the email address of the account they want to reset the password for (Fig 5.6). As the user hits enter, the KidFit API sends an access code to the email address provided and the user are presented with the next page where they enter the access code and the new password. If the access code entered by the user matches the one sent to the email address, the user password is updated in the database with the new password provided.

\subsubsection{Games View}

The games view presents the details of all the games that a user is involved in. At the top right, the page displays the name, number of steps and active minutes for the user 


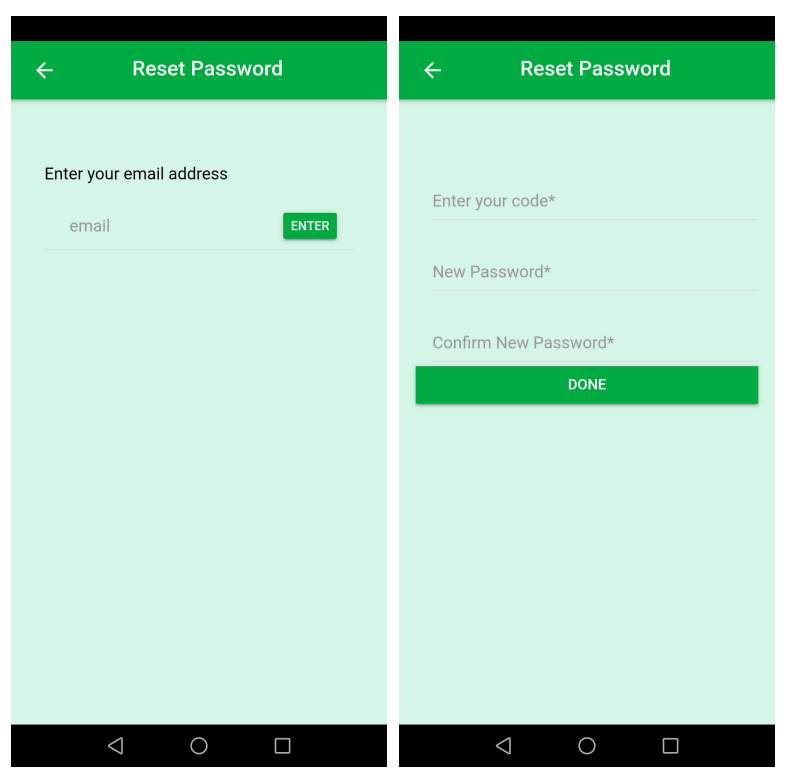

Figure 5.6: Forgot password view

(Fig 5.7). The text at the top of the screen briefly describe how to grow the garden (left) or capture the crown (right). The user can create a new game by clicking the button start new game (see Section 5.3.5). Invited games represents those games that the user is a part of and the game status in invited. The user can choose to accept or decline the games that they have been invited to using the green tick and cross (Fig 5.7 left). The opacity of the button to decline a game has been purposely reduced as that accepting the games and playing them is the primary action. The number beside each type of game invited, active and completed reflected the numbers of games respectively. The human icon on the right of each game, indicates the type of game. A single person icon represents a competitive game and a people icon represents a collaborative game. The active games tab indicates the active or ready to play games. The completed games tab tells the user the total number of games played and the number of games won. On clicking on the completed the games, the user can also see 
the activity they accumulated in the game (see Section 5.3.8). The refresh button at the top right, refreshes all the games and activity of the user. The logout button at the bottom logs a user out of the application. Since this is not a primary action, the opacity has been reduced.

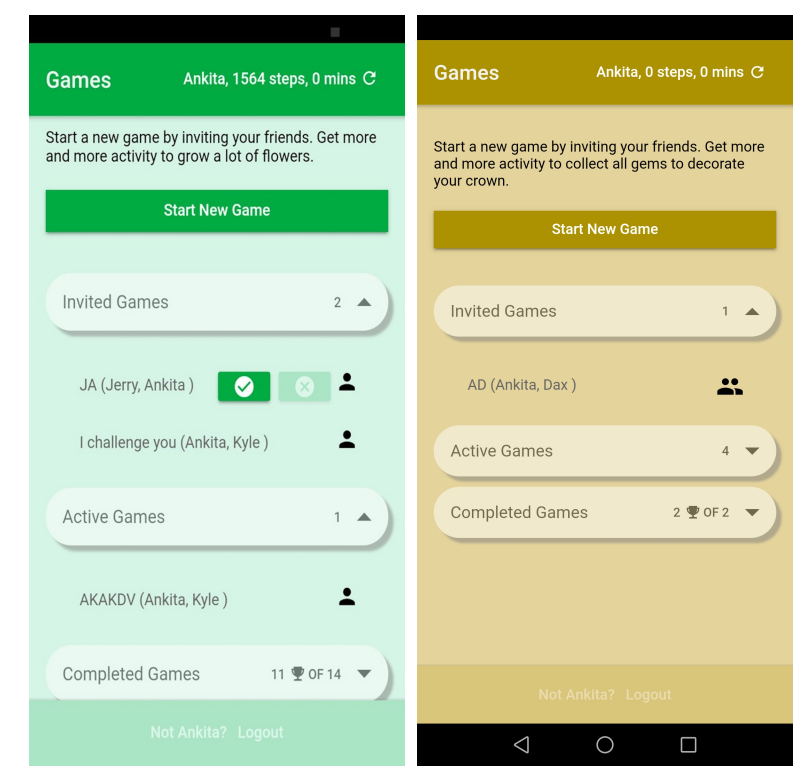

Figure 5.7: Games view (GTG on the left; CTC on the right)

\subsubsection{Create a Game View}

The start New Game button on the game page (Fig 5.7) lets a user create a new game with their friends (Fig 5.8). Visually consistent with the previous screen, the top right display the name of the user and their activity along with a button to refresh the view. The play in team and challenge others buttons let children decide the type of play as competition and collaboration respectively. Since the children were only allowed to play with other children in their group (see Section 6.1), they were presented with certain players to choose from. The tick boxes on the right could be clicked to select 
or unselect a player. Once the game is created, only the player who created the game has the authority to add or remove a player from the game. Children can decide a group name for the game. If the child doesn't enter a name for the group, the app provides them with a group name based on the initials of all the players. The New Game button creates the game.

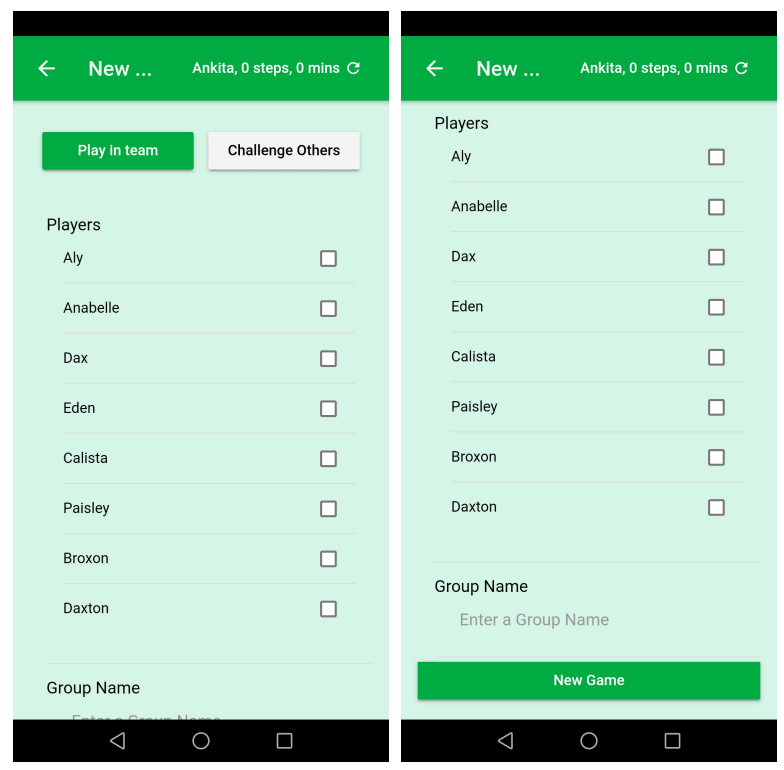

Figure 5.8: Create game view

Once the game is created, you can see the status (next to their names) of all the players in the game. The owner of the game, or the child who created the game can choose to invite more people or remove the ones in the game as long as the game has not started (see Fig 5.9 left). The players can also edit the group name any number of times. Ticking the check boxes to add or remove a player followed by clicking update button will update the game (see Fig 5.9 right).

If all the players in the game have accepted or declined the game invite such that the number of players in the game are two or more, such a game is ready to play and 


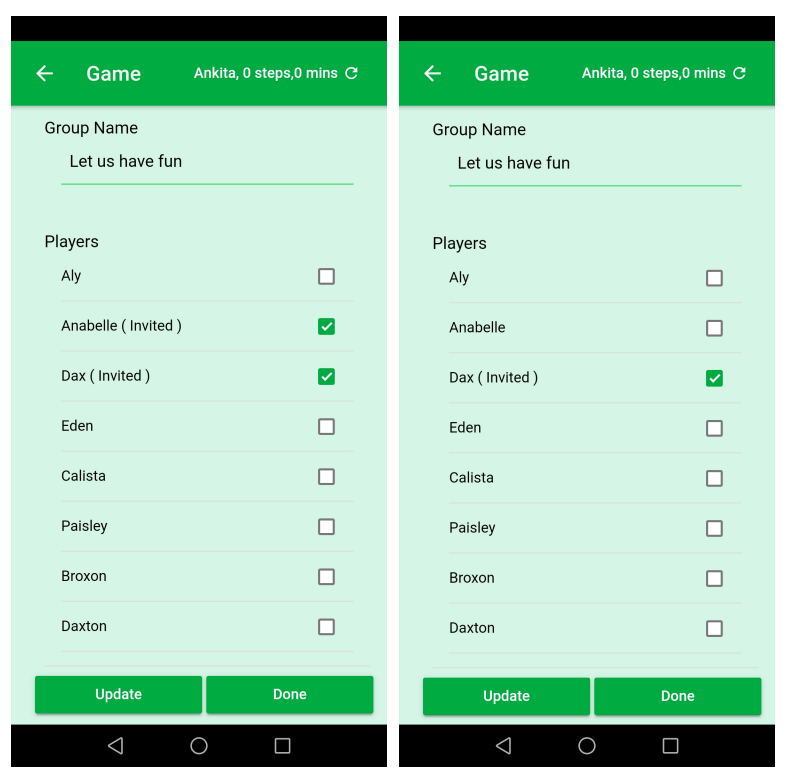

\section{Figure 5.9: Created game view (left); Remove a player (right)}

hence the view includes a start game button (see Fig 5.10). If a child starts the game, notifications are sent to all the other children in the game. If all the players declined the game request, or the owner deleted all the players, such a game can no longer be played as the game requires a minimum two people to play. In such a scenario the game status is set to quit game and the user can no longer access it.

\subsubsection{Competitive Game View}

As the user hits the start game button, the game starts. Based on the type of the game collaborative or competitive, the user is shown one or more gardens or crowns. Fig 5.11 shows the competitive type of view for GTG (left) and CTC (right). Since in a competitive type of play, users are competing against each other, the view shows the progress of each individual by their garden or crown. Consistent with the previous screens, the user's name and their fitness data are placed on the top right corner 


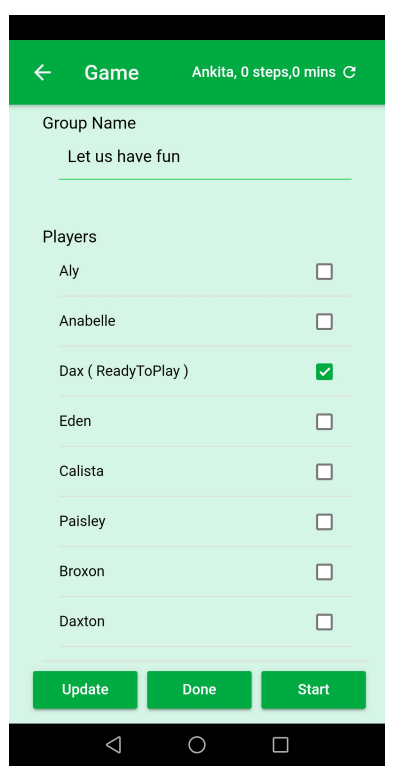

\section{Figure 5.10: Ready to play Game view}

along with a refresh button. The crown or the garden views for each child, shows their progress both in terms of visual flowers or gems and numbers (see Fig 5.11). The time on the top of the screen shows the time left in the game. As mentioned in section 5.1.2, the time for asynchronous game is longer and for a synchronous game is shorter. At the end of the timer, the player with maximum number of in-game steps (as indicated in the flowers or crown view) wins the game.

\subsubsection{Collaborative Game View}

Fig 5.12 shows the collaborative view for Grow the Garden (left) and Capture the Crown (right) respectively. As in collaborative type of play, children come together as a team, only one garden or crown that represents their cumulative activity is shown. The text on the screen informs the players of the remaining time and the target steps to achieve in that time. Unlike a competitive type of game, the user does not 


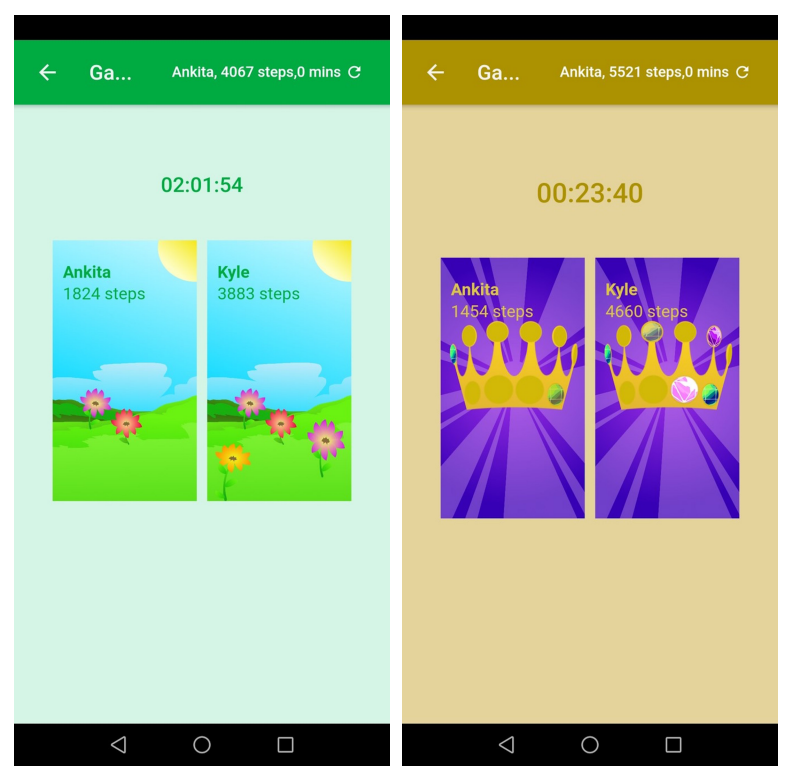

\section{Figure 5.11: Competitive game view GTG on the left \& CTC on the right}

know their individual progress to avoid any sort of competition in the game. The individual progress, however, can be known after the game ends (see Section 5.3.8). If the players are able to achieve the target activity before the time on the timer hits zero, the game ends indicating the team has won.

\subsubsection{Win/Lose View}

The end game of the screen tells the user their activity in the game, the flowers or gems collected and the status of the game and their status. A notification is sent to all the players in the game when the game ends. Figures 5.13 and 5.14 shows the win view and lost view of the game. 

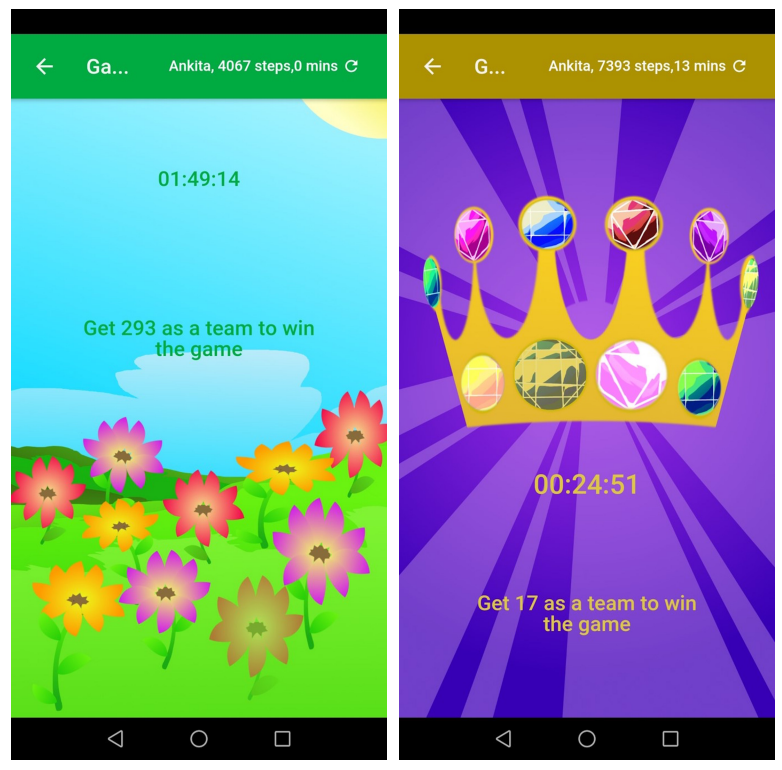

Figure 5.12: Collaborative game view GTG on the left \& CTC on the right
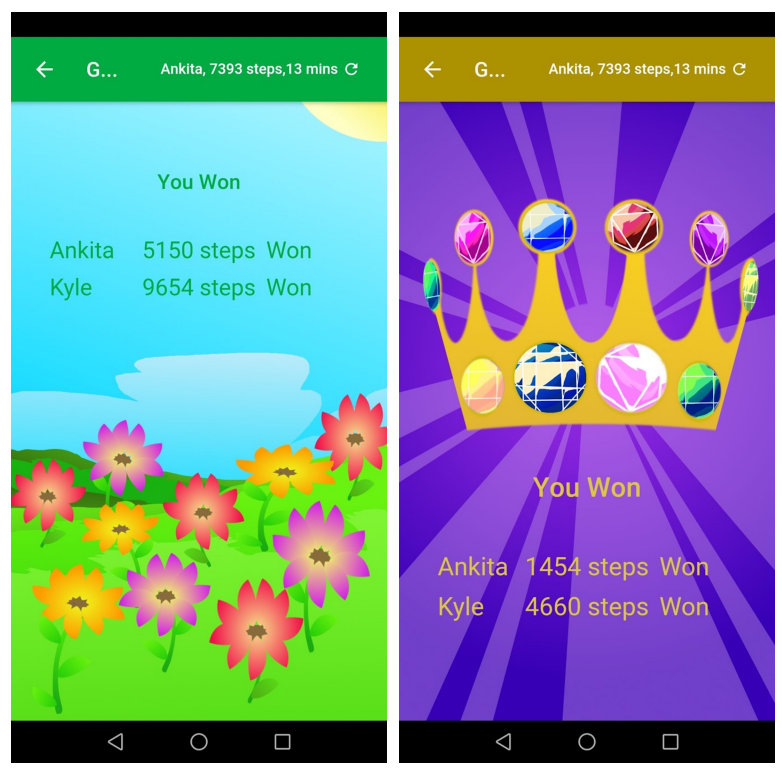

Figure 5.13: Win game view GTG on the left \& CTC on the right 

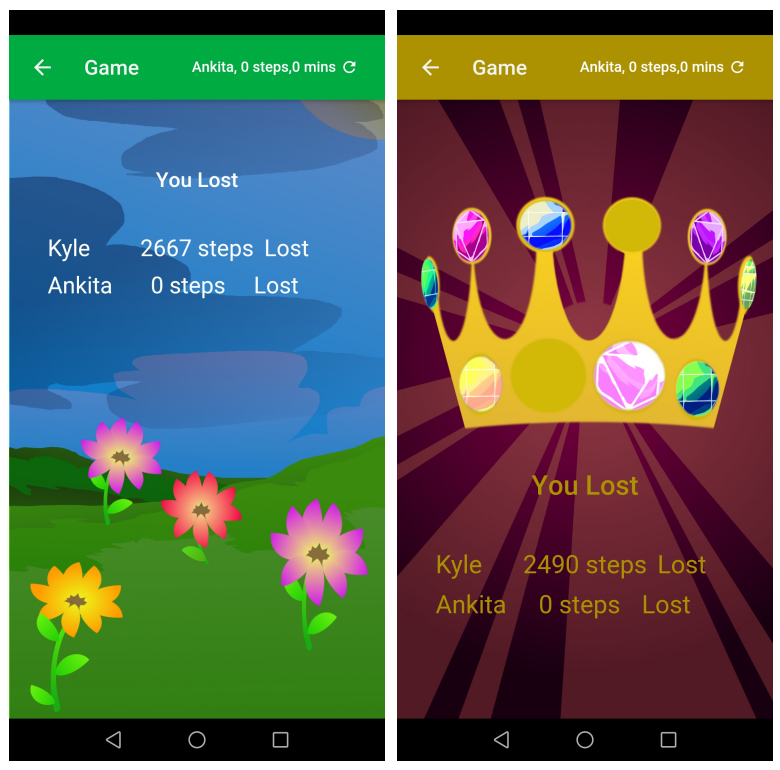

Figure 5.14: Lost game view GTG on the left \& CTC on the right 


\section{CHAPTER 6}

\section{INVESTIGATING SOCIAL AND TEMPORAL ASPECTS}

Having introduced the key elements in KidFit Suite, this chapter will elaborate on the observed and reported responses to the applications, their effect on children's activity levels, the contributing factors and more. The applications are primarily evaluated with regards to their social and temporal aspects and their effects. Approach, results, and limitations will be discussed for each of the application in order to provide a comprehensive understanding of the evaluation techniques and the resulting data.

\subsection{Method: User Study}

In order to test the effectiveness of these applications to promote physical activity in children I conducted a user study where 16 children (ages 6-11) played these games. This user study was submitted to and approved by the IRB in the form of an expedited review application. The participation in the study was voluntary and children were recruited via social media posts and word of mouth. The study was conducted over two one-week sessions followed by interviews with both children and parents. Parents were asked to take short demographic surveys after each week. In the first week of the study, children wore a Fitbit Charge 2 activity tracker and in the second week of the study, children wore a Fitbit Charge 2 and played the mobile applications Grow the Garden (GTG) and Capture the Crown (CTC) on an android phone that was 
provided. All of the other applications on the mobile phones except the calculator and calendar were password protected to avoid any distractions and to disable access to inappropriate content on the internet.

Table 6.1: Children's demographics

\begin{tabular}{|l|l|l|l|l|l|}
\hline Child No. & Parent No. & Pseudo Name & Age & Gender & Group \\
\hline C1 & P1 & Emma & 7 & F & G1 \\
\hline C2 & P2 & Jack & 9 & M & G1 \\
\hline C3 & P3 & Judy & 9 & F & G1 \\
\hline C4 & P4 & Kyle & 7 & M & G1 \\
\hline C5 & P5 & Maddy & 10 & M & G1 \\
\hline C6 & P6 & Matt & 9 & M & G1 \\
\hline C7 & P7 & Nate & 9 & M & G1 \\
\hline C8 & P8 & Nora & 9 & F & G1 \\
\hline C9 & P9 & Aly & 8 & F & G2 \\
\hline C10 & P10 & Anni & 8 & F & G2 \\
\hline C11 & P11 & Pearl & 11 & F & G2 \\
\hline C12 & P12 & Brody & 8 & M & G2 \\
\hline C13 & P13 & Daniel & 6 & M & G2 \\
\hline C14 & P14 & Dan & 6 & M & G2 \\
\hline C15 & P15 & Chloe & 11 & F & G2 \\
\hline C16 & P16 & Ella & 8 & F & G2 \\
\hline
\end{tabular}

Since the mobile applications are evaluated on synchronous versus asynchronous and collaborative versus competitive spectrums, Hot Potato was not included in the study. The reason for Hot Potato being excluded is that it can not be played in a collaborative manner as it is inherently competitive game and it can only support asynchronous play where not all children are active at the same time but take turns. The data from the first week of the study served as a baseline to get an understanding of the childrens normal activity levels (when not interacting with the games). This was then used to compare childrens activity data from the second week of the study where children interacted with the mobile gaming applications. The children were 
further divided into two groups of 8 children each, the first group referred to as Group 1 (G1) and second group as Group 2 (G2). Table 6.1 shows the demographic data of the children with their groups. The participants were grouped based on their preferences and availability to play the games. Children in G1 played GTG as short synchronous game and CTC as a long asynchronous game. This was reversed in G2 where GTG was played as asynchronous and CTC as synchronous type of play (see Table 6.2). The games, however, supported both competitive and collaborative game plays in each of the applications. The change in synchronous and asynchronous types of games in the two groups of children helped us understand any bias caused by the theme of gems or flowers on the primary spectrums along which I evaluate the applications. The applications only allowed participants to play with children in a given group and no one else. All the children in G1 had never met and did not know each other, while 6 of the 8 children in G2 were siblings or neighbours. Interviews with children and parents were conducted at the end of the second week. These provided qualitative responses to their (children and adults) perceptions about the games, activity levels, and problems they encountered.

Table 6.2: Overview of game configuration

\begin{tabular}{|l|l|l|}
\hline & Grow the Garden & Capture the Crown \\
\hline G1 & Synchronous & Asynchronous \\
\hline G2 & Asynchronous & Synchronous \\
\hline
\end{tabular}

\subsection{Key Findings}

This section presents the findings of the study from the interview responses and the activity data. The first section presents an overview of usage with demographics. The following sections elaborate on the social and temporal evaluations of KidFit Suite. 


\subsubsection{Overview of Usage}

This section starts with discussing the results and analysis of the fitness data from Fitbit, followed by an analysis of parents response to the demographic survey. The survey consisted of questions concerning the activity of children in the given week, how they utilized the tracker, the number of days they wore it and questions related to their perception as how was their child's activity in the last week differed from the normal. Out of the 8 children in G1, 3 children played 1 or less games. In order to not duly bias the data from G1, I excluded the data of those 3 children from the quantitative evaluations. However, their responses to the interviews helped us understand the problems they faced when interacting with KidFit Suite and hence is utilized in qualitative evaluations.

Some of the observation related to the number of games played by G1 and G2: a total of 107 games were created, 37 by G1 and 70 by G2. Out of these 107 a total of 68 games were played, 14 and 54 by G1 and G2 respectively (see Table 6.3). G1 played 9 collaborative and 5 competitive games, while G2 played 39 collaborative and 15 competitive games (see Tables 6.4 and 6.5). The average number of games played per child was six for G1 and twenty six for G2. The average number of days interacted with the app was three for G1 and close to six for G2. The difference in the interaction with the apps between the groups is significant. One of the reasons for a significantly higher number of games played by G2 compared to G1 could be attributed to the preexisting relationship and the proximity between the children in G2 (see Section 6.1). Other reasons can include, familiarity with the technology, weather conditions at the time of the study, school schedule, and better response from other players. Children from G1 interacted with mobile devices from Jan 3rd, to Jan 
9th which is a time of winter holidays in some schools. G2 played the games from Jan 14th to Jan 20th when the normal school session resumed. G1 had 17 games waiting for responses from participants while G2 had only 10. The higher number of invited games also indicate the lack of a medium of communication between children in G1 (also indicated in Table 6.14).

Table 6.3: Overview of games played

\begin{tabular}{|l|c|c|}
\hline & Games Created & Games Played \\
\hline G1 & 37 & 14 \\
\hline G2 & 70 & 54 \\
\hline
\end{tabular}

Table 6.4: Distribution of games played and activity collected by G1

\begin{tabular}{|l|l|l|l|l|l|l|}
\hline & \multicolumn{3}{|c|}{ Synchronous } & \multicolumn{3}{c|}{ Asynchronous } \\
\hline G1 & $\begin{array}{l}\text { No. of } \\
\text { games } \\
\text { played }\end{array}$ & Avg. steps & $\begin{array}{l}\text { Avg. } \\
\text { Active } \\
\text { min. }\end{array}$ & $\begin{array}{l}\text { No. of } \\
\text { games } \\
\text { played }\end{array}$ & $\begin{array}{l}\text { Avg. } \\
\text { steps }\end{array}$ & $\begin{array}{l}\text { Avg. } \\
\text { Active } \\
\text { min. }\end{array}$ \\
\hline Collaborative & 3 & 183.25 & 0 & 6 & 3134 & 9.9 \\
\hline Competitive & 3 & 2151.3 & 0 & 2 & 0 & 0 \\
\hline
\end{tabular}

Table 6.5: Distribution of games played and activity collected by G2

\begin{tabular}{|l|l|l|l|l|l|l|}
\hline & \multicolumn{3}{|c|}{ Synchronous } & \multicolumn{3}{c|}{ Asynchronous } \\
\hline G2 & $\begin{array}{l}\text { No. of } \\
\text { games } \\
\text { played }\end{array}$ & Avg. steps & $\begin{array}{l}\text { Avg. } \\
\text { Active } \\
\text { min. }\end{array}$ & $\begin{array}{l}\text { No. of } \\
\text { games } \\
\text { played }\end{array}$ & $\begin{array}{l}\text { Avg. } \\
\text { steps }\end{array}$ & $\begin{array}{l}\text { Avg. } \\
\text { Active } \\
\text { min. }\end{array}$ \\
\hline Collaborative & 27 & 6428 & 13.7 & 12 & 4808 & 11 \\
\hline Competitive & 12 & 3424 & 7 & 3 & 4136 & 0 \\
\hline
\end{tabular}

In terms of steps, children showed a difference in the average number of steps taken per day per child in week 2. G1 had the average of 7,729 steps per day during the study while G2 had an average of 10,614 steps per day (See Table 6.6). G2 also showed higher average steps collected in all games compared to G1 (see Tables 6.4 
and 6.5). All these facts indicate a bias between G1 and G2. Six children in G2 being familiar with one another could be one of the reason for this bias. Six children (not the same six children who were familiar with one another) in G2 had an average of more than 10,000 steps per day. Four of eight children showed an increase in average number of steps per day in G2, but none of the five children had an increase in steps in G1. However children in neither of the groups were active 60 minutes a day as reported by the activity data from Fitbit (see Tables 6.4 and 6.5). On some days even when some children had close to 10,000 steps or more, their activity minutes were still zero or very less (see highlights in Table 6.6), which indicate that the activity minutes reported by Fitbit might not be as accurate and hence may not be used to compute children's activity. 


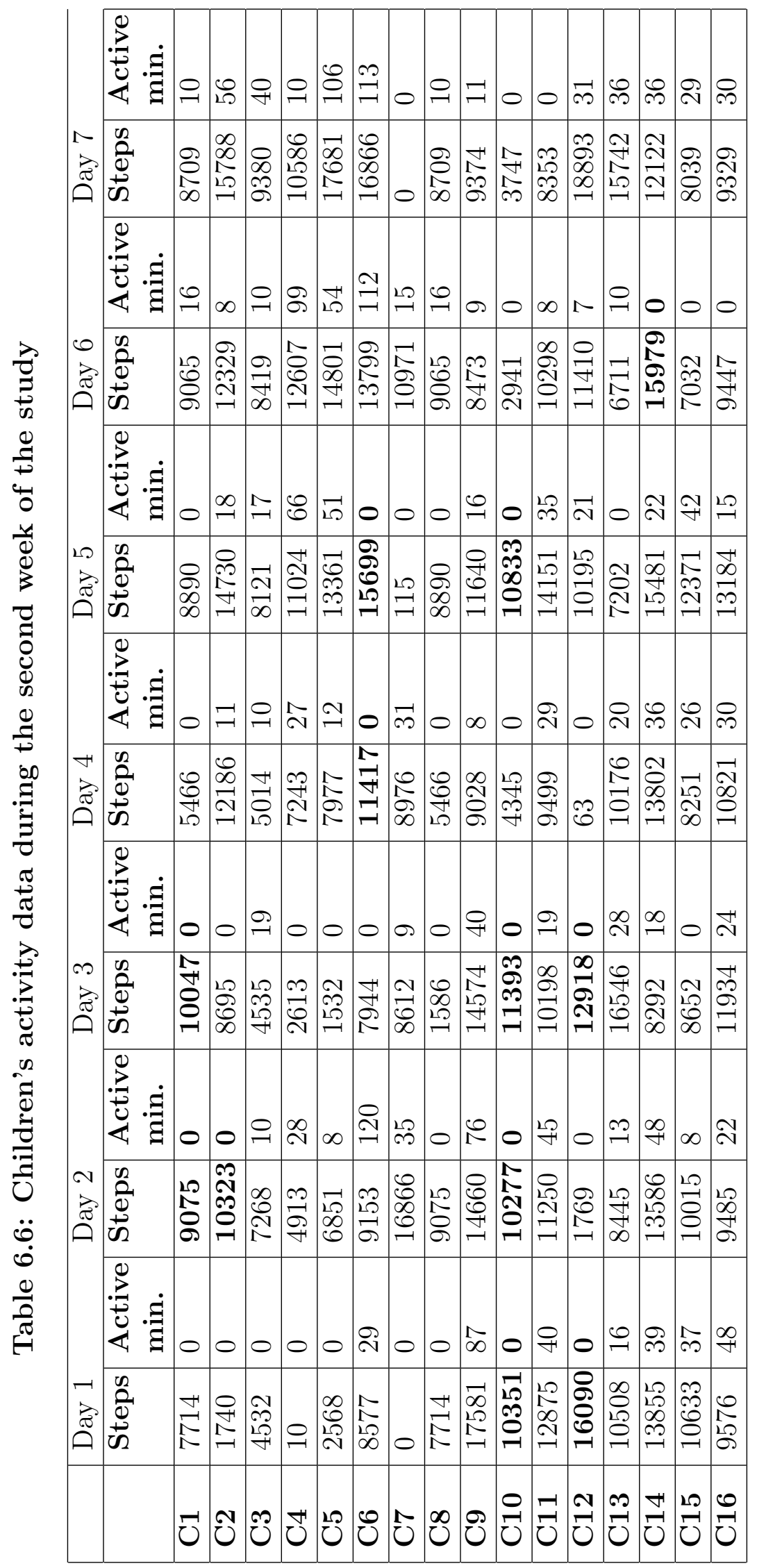


In terms of the visual appeal of the themes of flowers and gems, children showed preference to CTC. The number of CTC games (48) played are more than twice the number of GTG games played (21). However, the higher number of CTC games could also be the outcome of bias between the two groups as most of the CTC games (39) were played by G2. A parent also mentioned the theme of flowers seemed to be more intended to girls and the theme of jewels was more gender-neutral. In line with this, nine parents reported that their children preferred the theme of crowns, two parents said their children liked GTG while three were not sure if their children had a preference. Nine children reported liking the visuals of crowns and gems and four liked the garden better.

Another difference between the games played by the two groups show that only 2 (22\%) out of the 9 collaborative games in G1 were won while 30 (77\%) out of the 39 collaborative games were won by G2. As people are motivated by winning [26], the higher number of wins in the G2 may have promoted more participation.

From the 68 games played, 48 (70\%) games were collaborative team games and $20(30 \%)$ were competitive games. In terms of temporal preference in children, 45 $(66 \%)$ of games played were short synchronous games and $23(33 \%)$ were longer asynchronous games. The table below shows the distribution by category.

Table 6.7: Distribution of games played on social and temporal preferences

\begin{tabular}{|l|c|c|}
\hline & Collaborative & Competitive \\
\hline Synchronous & 30 & 15 \\
\hline Asynchronous & 19 & 5 \\
\hline
\end{tabular}

The demographics survey the parents took after each week, included a perception question "My child was more active than usual during the last week". In the interview they were also asked if they believed their child was more active than normal in a 
given week. Surprisingly, 6 of the 16 parents had contradicting responses to the two questions about week 1 (when children only wore a Fitbit) while 14 parents had agreement about week 2 (when they interacted with the mobile applications). From the detailed responses of the interviews, twelve parents mentioned that their children were more active than normal when just wearing the Fitbits. They said that the excitement of something new motivated them to reach the default 10,000 step goal to make the Fitbit vibrate. 15 of the 16 children had a daily goal to get 10,000 steps, while one was trying to exceed their steps from the previous day. This concurs from the findings of adults perceptions and children's fitness tracker usage survey, I conducted (see Chapter 3). Nine parents also indicated that their children were more active with the mobile applications than with Fitbits alone. Carrying a phone and knowing about their activity made children feel more confident. This may have been a possible reason for the increase in activity as reported by one of the parents.

\subsubsection{Social Element and its Impact}

The social aspect in the applications in KidFit Suite let children connect with their peers on the subject of physical activity. It enabled children (who were not necessarily co-located) to play together. Children had the flexibility to compete against their peers or collaborate with them.

Both children and parents liked the concept of combining physical activity and social interaction (see Table 6.8). Playing mobile games that let children play and connect with their peers, imitates natural childhood play. Some children liked competitive games better and some others preferred collaborative games. Parents reported

that playing with other children promoted friendships between the children even if they didn't know each other. However, they also said that it would be better if 
children could choose who they want to play with. In this user study, children could only play with the other children in their group. Children and adults indicated they would like to play these games with siblings, cousins, friends and family members(see Table 6.9). Challenging people (C11) you are familiar with, or teaming up with them would be more fun and motivating as one child reported. For children who do not have siblings and usually play by themselves, the mobile applications seemed be a platform to remotely connect and play with other children as answered by $\mathrm{C} 2$ (see Table 6.8).

The "little friendships" (P8) developing in the members of the group was also evident from the group names (see Table 6.10). Children would make funny group names but they would make sure that the names are respectful and motivating for other children. As two of the children said:

I liked to name the group and change the group name because one was 'Jack and dumb', and I wanted to change it, so I changed it to 'Jack is something' and that was crazy. It shouldn't let you put dumb in any of the group names. $-\mathrm{C} 13$

I noticed when I was playing other people, one of them made a nice name, it was like good job buddy something like that. So I felt like when I would play the games, other people would not just make me names and stuff. They would make name that like cheer on the other players. - 66

All the parents said that they would like to play such games too. They appreciated the games stating that it was good, that the games could be played indoors too, 


\section{Table 6.8: Children's and parents' views related to the social element in the applications}

C16 I like them. they are fun and its fun to be just competitive and not have to be in the presence of another person.

C2 More active. because I had other people to play with. instead of just like myself

C2 I liked that instead of just like running around, you get a like, you can see how many steps you get and stuff like that. It doesn't just like not count your step. It counts every single step, not like every other one. I liked how you got to play with other people, like you would be in teams and challenge other people.

C15 I was more active with the phone because I'm super competitive and its against my friends which makes me more competitive and my siblings.

C11 I prefer Capture the Crown over Grow the Garden. I like them because I could challenge my siblings and my friends or neighbours.

P8 It's more of an incentive than to just go out and be active when you get to tell so. She said she thought it would be fun to meet the people that she's challenging. And so I think it, Causes a little little friendship even though she didn't know anyone. They would call their teams funny names and she liked doing that with whom would you like to play. I'd like playing with her

P10 I like the idea of it being fitness based and incorporate it into technology because that's just what kids are doing these days. And. I like the challenge. The challenges and then the ability to play with other kids and have two choices They can choose how many. And so they just really feel in control of it and it's theirs.

P13 Oh I think yeah. I think having like an indoor especially in the winter something indoor to get everyone active. I think that would be really fun. I think its just me like it's you'd feel really silly running around your living room but with a game in your hand you don't feel as silly. So it's a good exercise for them and they would, they would like it because they got really excited when people were playing 
Table 6.9: Children's and parents' responses related to who would they like to play with

\begin{tabular}{ll}
\hline C15 & I would play with my friends. I would drag my friends into this. \\
\hline C6 & My art Teacher and my PE teacher \\
\hline C13 & my siblings and parents \\
\hline C14 & my family and sometimes with my teacher \\
\hline P9 & $\begin{array}{l}\text { Me and my sister kind of gravitate to the same thing so she might } \\
\text { join in with me if I brought it up to her. Normally I don't play } \\
\text { any games on my phone or anything like that but if it's towards } \\
\text { health or bettering yourself or anything like that that's that always } \\
\text { interests me. }\end{array}$ \\
\hline P2 & $\begin{array}{l}\text { Yes. His friends from school and family. It would get the family } \\
\text { more active as well and friends }\end{array}$ \\
\hline P13 & It would be fun if they could play like with cousins and stuff. \\
\hline
\end{tabular}

especially in the winter. As one of the parents (P13) said that this could be something the entire family can do together. They said, it might feel silly when you are running around in your living room, but not so much when you have a phone in your hand and you get to do it with your family.

Table 6.10: Motivating group names created by children

\begin{tabular}{|l|}
\hline We 3 awesomes \\
\hline Morning Game! \\
\hline Play Now! \\
\hline WIN this round \\
\hline Hurry up \& see \\
\hline Fun Team \\
\hline
\end{tabular}

The ability to socially connect with children as you play with them was reported to be motivating (see Table 6.11). Children believed playing with peers encouraged them. They even understood that the team games had a higher step goal and would still try to achieve it (C14). Parents reported that the games added 
an incentive of social interaction to physical activity. They said that children behaved more responsibly by being active in a team game, so that other members of the team don't lose the game. One child (C11) had a goal to do more steps than the previous day. The motivation in one of the children (as reported by P6) was also noticed by their therapist (see Table 6.11). As reported by one parent (P3) "It's nice to have something to motivate you to be active at home". Children thought it would be fun to meet the people they are playing with. The games also informed children of their activity levels and they felt more confident about themselves. One parent (P5) reported that the applications helped them better understand their child's current activity levels and how he needed more activity than he usually does. The motivation and confidence in children can also be witnessed by the fact that 11 children believed they were more active than normal while playing the games.

As mentioned earlier, $70 \%$ (48 of 68 ) of games played were collaborative team games while only $30 \%$ (20 of 68 ) of games were competitive games. This was also consistent with the interview responses where 8 children mentioned preferring team games, while 3 of them said, they liked challenges better, the rest were not sure if they liked one better than the other. The higher number of collaborative games may also indicate a preference towards team games in children. However, since most of these collaborative games (39 of 48) were played by children in G2, there could be a bias introduced by the grouping of children. Table 6.12 lists some of the responses of parents and children about Collaborative games.

Another reason that may lead to children leaning to collaborative games might be, that they win or lose together as a team. As in collaborative games, all the players work together to achieve a common goal, it seemed easier to win. In fact $68 \%$ (32 of 48) of team games were won. One child (C15) thought that they always win in 


\section{Table 6.11: Children's and parents' responses related to social motivation of the applications}

\begin{tabular}{ll}
\hline C3 & Because during the Fitbit thing it was just like showing what I \\
am without it. And with the games I could challenge, it would \\
challenge me to run more in and walk more and all those things.
\end{tabular}




\section{Table 6.12: Children's and parents' responses related to collaborative game play}

C1 Team games, because you get more steps and like it adds up like somebody has a ten thousand steps and somebody has ten thousand steps you get twenty thousand steps like that is something big. So I would like that.

C3 Team. because it included other people and it wasn't turning against other people and being like I don't like you. because it had a shorter time like I mentioned before

\begin{tabular}{ll}
\hline C14 & $\begin{array}{l}\text { I just liked that we can work on it as a team. I like working as a team } \\
\text { because then its more likely that you will actually do something and } \\
\text { it gives a little more encouragement as it has a higher step goal for } \\
\text { the team }\end{array}$ \\
\hline C16 & I liked that usually you work as team and they gave you more time. \\
\hline C15 & team games because you always win. \\
\hline C12 & $\begin{array}{l}\text { Team because it seems like that you win more when you are on } \\
\text { a team but its not as fun. Its just kind of more interesting Liked } \\
\text { the short timing and jewels. its exciting because you have short } \\
\text { amount of time to get a lot of steps. }\end{array}$ \\
\hline C13 & $\begin{array}{l}\text { I liked how you were working as a team instead of racing against } \\
\text { people because you didn't have to do it all by yourself. }\end{array}$ \\
\hline
\end{tabular}

a team game. Another child believed it was hard to play a challenge as others got many more steps than they did so they preferred team games. As shown in Table 6.12 , children were also motivated by winning and picked the game they thought was easier to win. Some others believed that challenge games were like turning against other people and not liking them (see Table 6.12). Children who liked competitive games said that they were more active because they wanted to beat everyone and win the game (see Table 6.13).

While the number of collaborative games played was higher and were preferred by more children, some children liked challenges more. Challenges were preferred 
when the children knew each other as in G2 (see Table 6.13). Challenging siblings or friends was not seen as turning against them or not liking them, rather it was seen as a healthy competition. In fact, 15 competitive games were played by G2 compared to only 5 (two of the five games had no in-game activity) by G1 (see Tables 6.4 and $6.5)$.

Children also somehow assumed a correlation between competitive and short synchronous games though none was mentioned. They believed that if you are competing against someone, you shouldn't have to do it for long and hence shorter games seemed more competitive. One of the children suggested that CTC (synchronous play in their group) should only support competitive game while the GTG (asynchronous play) seemed more collaborative as you have a longer amount of time to reach the goal (see Table 6.13).

\section{Table 6.13: Children's and parents' responses related to competitive game play}

C16 I like them. they are fun and its fun to be just competitive and not have to be in the presence of another person.

C13 I like how it was a shorter time so if you were doing it in like a competition then you didn't have to do it for as long.

\begin{tabular}{ll}
\hline C4 & I liked how it was shorter so it was more competitive \\
\hline C15 & $\begin{array}{l}\text { I was more active with the phone because I'm super competitive } \\
\text { and its against my friends which makes me more competitive and } \\
\text { my siblings }\end{array}$ \\
\hline C14 & $\begin{array}{l}\text { Capture the Crown because it was more competitive than Grow the } \\
\text { Garden because it had less time }\end{array}$ \\
\hline P11 & $\begin{array}{l}\text { As I had one she was motivated to challenge me. She was always } \\
\text { asking me how many steps do I have and trying to see how she was } \\
\text { beating me/not and if she was not beating me she wanted to go do } \\
\text { more steps. }\end{array}$ \\
\hline
\end{tabular}


The applications required more than one child to play the games. To create a game, a user invites one or more children to play along. In the current implementation the game cannot start, until all the players in the game have responded to the invitations. This was a major challenge faced by the children while interacting with the applications. As the applications did not support notifying the user on receiving an invite, and because they didn't always have their phone with them, children did not know of invited games until they checked the app. They also had to wait on the response from all the players to get started with the game. This issue was frequently reported by both parents and children (see Table 6.14)

As the children in G1 were not acquainted, they were not aware of each others schedule and hence starting the games was a bigger challenge for them. Six Children in G2, however, were well aware of each others schedule and could communicate (as they were co-located), making it easier to start the games for them. This is also evident by the number of games played by each group and the number of days interacted with the applications. Fewer games on GTG were also indicated as a result of children not responding to that game (see Table 6.14).

To overcome this problem of waiting for invitations to be accepted, children suggested that it would be good if they could know who is available to play and invite them for a game (see Table 6.15). Other suggestions included better notifications for game invitations and game starting (see Table 6.15).

\subsubsection{Temporal Flexibility and its Impact}

The flexibility to choose from short or long games was appreciated (see Table 6.16). Children would decide to go out and run based on the time left on the game. 
Table 6.14: Children's and parents' responses related to fewer GTG games

\begin{tabular}{ll}
\hline C5 & No one really accepts my games on that (GTG).I kind of liked \\
& crowns better. I like longer games. \\
\hline C9 & because for some reason there were more games that won't start on \\
& that one (GTG) \\
\hline C8 & it took a while for people to respond \\
\hline C7 & sometimes people would not respond and it would take a long time \\
& to start. there were certain people who did not respond that many \\
& times and certain people respond more often. \\
I didn't kind of like that not that many other people would play it, & nobody would accept the request \\
\hline C11 & didn't like Grow the Garden because it just wasn't played as much, \\
& it wasn't popular \\
\hline P6 & I didn't like that people didn't respond. And it should be like I don't \\
know it seems like they could respond but then nobody started the \\
game. I don't really understand. It just seem like I don't know how \\
you could make it more mandatory that they have to if they're if \\
they're agreeing to be in the group then they should agree really \\
to play.And it's a little bit because there's no communication If \\
somebody accepts then.
\end{tabular}

Table 6.7 indicates that short synchronous games were played more compared to longer asynchronous games. Children's response to their choice of preferred games between the two games also concurs with the table. Three of five children in G1 preferred GTG (synchronous play for G1), while seven of eight children in G2 liked 


\section{Table 6.15: Children's and parents' suggestions related to streamlining game invitations}

\begin{tabular}{ll}
\hline C5 & It should give you a notification on the watch when a game starts. \\
\hline C6 & $\begin{array}{l}\text { Maybe like, saying this person isn't logged on, please login again. } \\
\text { because it didn't tell you and I got confused. }\end{array}$ \\
\hline P9 & $\begin{array}{l}\text { Maybe making it possible where you can disinvite people even if } \\
\text { you didn't create it just so that you can get the game on }\end{array}$ \\
\hline P10 & $\begin{array}{l}\text { I think it would be a fun idea. But it need to be instantaneous as far } \\
\text { as like people join and its within a couple minutes of people joining }\end{array}$ \\
& and all the data needs to be synced seamlessly into the game. \\
\hline P11 & $\begin{array}{l}\text { Well people just need to know when it's starting. So if it was on the } \\
\text { situation where it was live and it was the whole world was playing }\end{array}$ \\
& $\begin{array}{l}\text { you'd be instantly like. People would sign up within a minute or } \\
\text { two of each other and you'd all play at the same time and it would } \\
\text { be really easy and succinct. }\end{array}$ \\
\hline
\end{tabular}

Table 6.16: Children's and parents' responses related to temporal Flexibility in the applications

\begin{tabular}{ll}
\hline C3 & More active. Because like I'd see how much time I had and my plan \\
to go take a run around the block or something.
\end{tabular}

CTC better (synchronous play for G2). Since synchronous games were short (2545 minutes), they seemed more achievable to some children. On the contrary as asynchronous games were longer (2.5-6 hours), children believed they are a lot to do. Two of the children perceived that they had to be active the entire time in the game and hence did not prefer asynchronous games. Again, since the children in G2 resumed normal school activities at the time of study, asynchronous games were found to be harder for them to play given the longer game times and their school 
schedules.

Children related short synchronous games to competitive games, and longer asynchronous games to collaborative games. One possible reason for this could be the higher step goal in the longer, collaborative games which was to be achieved by children as a team and not as an individual goal. Though, the step goal for synchronous collaborative games is higher than competitive games, it is not as high as asynchronous collaborative games. In fact, children who preferred competitive games, indicated a preference towards synchronous games too saying that they feel more competitive and intense when the time in the game is short. Children also related that shorter time in the game times motivated them to be active (see Table 6.17). One child (C3) indicated that more time in the game made her procrastinate her activity, while shorter time encouraged her to do it, then and there.

Though I did not specify explicitly, children's assumption of being active for the whole time, dominated their preference of temporal flexibility. The bias in groupings may have further contributed to the understanding of asynchronous games and playing them. As children in G2 indicated a preference towards the theme of CTC which was a synchronous type of play for G2, that might be one of the factors contributing to higher number of synchronous games being played.

Though the interview questions did not directly ask about synchronous or asynchronous games, children mentioned choosing the games according to various preferences. For example, they mentioned choosing the in-game activity based on the time in the game (see Table 6.18). Some children said they liked longer games, as you have more time to accomplish your goal especially when in teams. One child (C3) liked that it let them take little breaks in between each run, which was the motive behind temporal flexibility. Another (C12) child believed more time enabled them to get a 


\section{Table 6.17: Children's and parents' responses related to short synchronous games}

\begin{tabular}{ll}
\hline C3 & Like it had a shorter time than Capture the Crown and made me \\
& want to run more instead of like knowing I had this much time I go \\
& I can do this first instead of running now. \\
\hline C12 & CTC because it was more competitive than Grow the Garden \\
& because it had less time \\
\hline C13 & $\begin{array}{l}\text { I like how it was a shorter time so if you were doing it in like a } \\
\text { competition then you didn't have to do it for as long. }\end{array}$ \\
\hline C14 & I liked how it was shorter so it was more competitive \\
\hline C15 & CTC challenge, Its easier as its a lot shorter and its a lot more \\
intense & $\begin{array}{l}\text { I liked that it was shorter. So that was a lot easier because you } \\
\text { didn't know when it was going to start and it's like you know Oh } \\
\text { shoot it's a two it's not like a two hour game that starts at 9:00 } \\
\text { at night. So forty five minutes was a little bit more like you know } \\
\text { doable and I like the visuals of the crown jewels. }\end{array}$ \\
\hline
\end{tabular}

higher number of steps (trying to reach the 10,000 steps daily target). One other $(\mathrm{C} 4)$ believed that you can only get a lot of steps in a short amount of time only if you are very athletic or active (this same child, did not prefer competitive games indicating that others were able to get many more steps than him).

Both children and parents suggested that it would be good if they had the flexibility to choose the duration of the game. This would facilitate children to not choose one of the themes based on the timings or vice-versa. One child (C9) said:

probably it could let you select a time say 3 or 4 hrs. it shouldn't give you less time.

Allowing users to select the game duration may enhance the flexibility provided by the temporal aspect but may deter engagement with the applications if the duration 
Table 6.18: Children's and parents' responses related to long asynchronous games

\begin{tabular}{ll}
\hline C3 & $\begin{array}{l}\text { I liked that. It let me have little breaks in between each run, things } \\
\text { like that. }\end{array}$ \\
\hline C7 & I like longer games. \\
\hline C12 & It gave you more time to get more steps. \\
\hline C13 & GTG because it wasn't as competitive as it had more time. \\
\hline C14 & $\begin{array}{l}\text { I liked that you had more time than Capture the Crown to get more } \\
\text { points. }\end{array}$ \\
\hline C16 & $\begin{array}{l}\text { I liked that usually you work as team and they gave you more time. } \\
\text { P4 }\end{array}$ \\
$\begin{array}{l}\text { I think that the only thing that could be improved probably is } \\
\text { maybe longer times so rather than the 30 minutes give them a } \\
\text { couple of hours I know a couple of the games did and then some } \\
\text { didn't. }\end{array}$ \\
$\begin{array}{l}\text { That was the one where they had to do the two hour. That one I } \\
\text { felt Was a little more frustrating because they had a lot of kids on } \\
\text { there and they had kids that never responded. }\end{array}$ \\
\hline P11
\end{tabular}

selected by one of the players is not suitable for other players in the game. Besides, since the target activity to win the game is decided on the duration of the game and the number of players, selecting a very long time may result in an even higher step goal which might cause amotivation in children.

\subsection{Discussion}

The idea of leveraging technology to promote physical activity was very well received and appreciated. As one of the parents (P8) said "I like the idea of it being fitness based and incorporates technology because that's just what kids are doing these days. And I like the challenge. The challenges and then the ability to play with other kids and have two choices. They can choose how many. And so they just really feel in 
control of it and it's theirs". The fondness for the applications was also evident by the fact that all of the children and the parents in the study said they would like to play more such games in the future. Some of the children (who had access to mobile phones with internet) tried searching these games on the internet so that they could continue playing them even when the study was over. Some others were sad about giving them up at the end of the study. One Parent (P15) said that though their child was excited about Fitbit initially, but with the games, they forgot about it and instead, were busy playing the games. Having the flexibility to choose who the children wanted to play with rather than just the children in their group was one suggestion that was persistent for all the players. The applications (as reported) seemed to promote friendships in children apart from from encouraging them to be physically active. The theme of CTC was liked more as it seemed more gender neutral while GTG was believed to be designed keeping girls in mind. Children said that they found the crown more dazzling like they are actually collecting a treasure. They were motivated by the games and gained confidence in their activity. The themes, content and the complexity of the applications was reported to be "cute and age-appropriate" (P14) while one child (C7) thought they were childish. The applications also helped parents get an understanding of their child's activity levels. One parent also thought that interacting with the applications was learning for the child of how the phone and the Fitbit interacted. Two children however said that they were more active without the application as they were distracted looking at the flowers, or jewels and the time left on the phone/in the app.

A rather disappointing result from the study was that none of the children were able to meet the activity guideline of 60 minutes per day (as calculated from Fitbit activity data). Although twelve parents believed that their children were able to get 
to 60-minutes of activity each day. One of the parents said "Well I still believe she's active for 60-minutes although that's not the same definition that Fitbit has". From the Fitbit activity data, even though the children had close to or more than 10,000 steps on some days, their activity minutes still showed zero (see highlights in Table 6.6). This mismatch between steps and active minutes was surprising to find and raises the question of whether the 60-minute activity guideline for children should be calculated using activity minutes in fitness trackers or not.

As children accumulated close to 500 steps, flowers or gems start showing up on their screen. This visual feedback was found motivating and encouraged children to win the games. Winning the games was also a driving factor for choosing one. Parents reported that children preferred the games they thought they would win. Few children thought it was easier to win team games and hence preferred them. Some parents suggested that the step goals may be high for the younger ones and reducing it a little might help them win and hence play more. Winning and losing the games were both liked by one child (C12), as he said you get to keep the crown even if you lose (see Fig 5.14). 


\section{CHAPTER 7}

\section{FUTURE WORK \& CONCLUSION}

This closing chapter first contains future improvements for this work and closes with conclusions drawn from this work.

\section{1 $\quad$ Future Work}

Though KidFit Suite was well received by the participants in the study, there were a few limitations that will need to be addressed as part of the future work. This section discusses those limitations and the future work.

\subsubsection{Simplify Activity Measures}

As observed from the children's fitness tracker usage survey (see Chapter 3), children tend to follow adults and used steps to set their goals. Similar observations were seen in this study where 15 of 16 children reported their daily goal to be 10,000 steps or more. The recommended activity guideline for children suggests 60-minutes of activity each day. However, since Fitbit does not report and update active minutes until there is a contiguous block of activity greater than or equal to 10 minutes, I used steps as a measure of activity. This enabled us to provide children with immediate feedback. Also, the mobile applications in KidFit Suite utilized fitness data provided by the Fitbit server. As mentioned in Section 5.2.2, fetching fitness data from Fitbit, 
burdens the user with the overhead of having to synchronize data with the Fitbit app in order to get accurate data on their KidFit apps. This issue was also raised by some of the users who said that remembering to synchronize the data with Fitbit app was cumbersome. It also affected the user experience, such that, even if a child had synchronized their data, the game would not end until all the players in the game had synchronized their data. One parent suggested that the data synchronizing should be seamless (see Table 6.15). To address these concerns and simplify the activity measures, the future implementations of KidFit Suite should work towards incorporating fitness data from built-in activity trackers in mobile phones. This will not only avoid the dependence on Fitbit data, but will also reduce the need to wear an additional device.

\subsubsection{Add a Medium of Communication}

When a child creates a new game, they invite other children they want to play with, and wait for them to respond. This waiting for invitation responses was a major issue faced by the children. As the applications did not notify the children of a game invitations or on a status change for a game, they encountered issues starting a game, waiting for the response from other users (see Table 6.14). Parents also indicated that the applications lacked a medium of communication with other children which could facilitate letting them know of the game invitations. Future versions of KidFit Suite could incorporate a chat server which would facilitate two purposes: first, it will enable richer communications and hence enhance their social experience and, second help streamline the game invitation process. 


\subsubsection{Address Logging Out of the Application}

Several parents from the user study reported the issue of children occasionally unintentionally logging out of the applications on pressing the back button. In order to log back in, they had to use an email address and a password which may be hard for younger children to remember. This problem was identified, and resolved such that the applications no longer logged a user out until they selected the log-out button on the games view.

\subsection{Conclusions}

I have introduced KidFit Suite, a collection of mobile applications. The final study presented in this thesis used to investigate social and temporal aspects was conducted with 16 children over two one-week sessions. Children wore a Fitbit Charge 2 activity tracker in both weeks and played the games in KidFit Suite in the second week. The fitness data from the first week of the study was used a baseline to compare data from the second week of study where children interacted with KidFit Suite. Data from Fitbit activity trackers and interviews were used to analyze children's and parents' response to this applications and the motivations or encouragement brought

about by KidFit Suite. The children were further divided in two groups of 8 children each referred as G1 (group 1) and G2 (group 2).

\subsubsection{Steps Increased in G2, Activity Minutes Did Not}

The idea of leveraging technology and games to promote physical activity was well received and appreciated. As six children in G2 were familiar with each other, there was a bias in the two groups. There was more interaction with the apps in G2 
compared to G1. The average steps per days was even found higher in G2 (10,614 steps) compared to G1 (7,729 steps). Though we cannot say affirmatively, the higher interaction with the apps may have been one of the contributing factor to the higher activity in G2.

Sadly, children in neither of the groups were active 60-minutes a day as reported by the FitBit data. However, on a few days even if they had 10,000 steps or more, their active minutes were close to zero, indicating that the active minutes as reported by FitBit might not be accurate to use for analyzing children's activity.

\subsubsection{Visual Preference for CTC}

Children preferred the theme of crowns as is evident by the number of games played (47 CTC games and 21 GTG games). The theme of CTC was described as if collecting a treasure of gems and was reported to be gender-neutral.

\subsubsection{Motivation to Win Led to More Collaborative Games}

Winning the game was also seen as a motivation to choose which game to play. This was also reported by some child participants. In terms of games, 77\% (30 of 39) collaborative games were won by G2 while only $22 \%$ (2 of 9 ) collaborative games were won by G1 hence higher number of collaborative and overall games in G2.

\subsubsection{Impact of Social Interaction: Collaborative Preferred}

Both children and parents liked the idea of bringing social interaction with physical activity. As indicated from the number of games played, and the responses to the interview, both collaborative and competitive games were liked by children (see Table 6.12 and Table 6.13). Children showed a preference towards collaborative games 
over competitive games such that $70 \%$ (48 of 68) games played were collaborative games. They were aware of the higher step goals in collaborative games and were still motivated to achieve it. However, a higher number (48) of collaborative games may also be linked to the theme of crowns, the motivation to win easier in collaboration, and the bias between the two groups.

\subsubsection{Impact of Temporal Flexibility: Synchronous Preferred}

The data from the survey, the mobile applications and interviews showed that the Temporal flexibility in the applications to choose the duration of the game was also beneficial. Based on their personal preferences, some children liked synchronous games while others liked asynchronous type of play. However, from the number of games played and responses of the interview the children seemed to have a preference towards shorter synchronous games as $66 \%$ (45 of 68) games played were synchronous. Because of the shorter duration of synchronous games, they seemed easier to play and more competitive.

\subsubsection{Social and Temporal Correlations}

Children linked short synchronous games to competitive games and longer asynchronous games to collaborative games. They suggested that one of the games (GTG or CTC) should only be short and competitive, while the other one should be long and collaborative.

\subsubsection{Overall Response}

The games were liked such that all 16 of the children and their parents reported that they would like to play such games in the future. One child also tried to find these 
applications on play store to continue playing them after the study was over. Parents saw these games as something the entire family could do together, even during the winters.

\subsection{Revisiting Thesis Statement}

The social interaction of collaborative and competitive experiences enabled children to connect with their peers while being active and was reported to be motivating. Collaborative games were preferred over competitive games as children perceived it was easier to win them and they were viewed as more prosocial. Competitive games were liked when children were familiar with each other and were not seen as turning against them.

The temporal flexibility of synchronous and asynchronous experiences enabled children to choose the duration of the game and children reported that they would plan their activity according to the time left. Short synchronous games motivated children to be active and were reported to be more competitive. Longer asynchronous games were preferred by children who thought it was hard to get a lot of steps in a short amount of time.

The findings of this research contribute to better understanding the elements that make physical activity fun and playful for children. 


\section{REFERENCES}

[1] FitQuest Lite An App for Reaction Time Practice. Retrieved March 21, 2019 from https://starfishtherapies.wordpress.com/2012/03/28/ fitquest-lite-an-app-for-reaction-time-practice/, March 2012.

[2] How much physical activity do children need? | Physical Activity | CDC. Retrieved Dec 9, 2018 from https://www.cdc.gov/physicalactivity/basics/ children/index.htm, 2012.

[3] Active minutes. Retrieved March 25, 2019 from https://support.mymotiv . com/active-minutes-S1uemFswZnZ, 2018.

[4] BMI Calculator Child and Teen | Healthy Weight | CDC. Retrieved Sept 18, 2018 from https://www.cdc.gov/healthyweight/bmi/result.html? \&method=english\&gender $=f \& a g e_{-}=11 \&$ age_m=0\&hft $=5 \& h i n=5 \& t w p=150, \quad A u-$ gust 2018.

[5] Commentary: How the New Fitbit for Kids Could Backfire | Fortune. Retrieved April 18, 2019 from http://fortune.com/2018/03/22/ fitbit-ace-kids-children-eating-disorders/, 2018.

[6] Fitness Trackers for Kids. Retrieved April 18, 2019 from https://www .eatright.org/fitness/exercise/family-activities/ fitness-trackers-for-kids, 2018. 
[7] Global wearables sales by category 2014-2017 | Statistic. Retrieved Sept 20, 2018 from https://www.statista.com/statistics/757623/ wearables-sales-by-category-worldwide/, 2018.

[8] Childhood Overweight and Obesity | Overweight \& Obesity | CDC. Retrieved April 5, 2019 from https://www.cdc.gov/obesity/childhood/index.html, February 2019.

[9] Fitbit Development: Fitbit Web API Basics. Retrieved March 26, 2019 from https://dev.fitbit.com/build/reference/web-api/basics/, 2019.

[10] What is Childhood Obesity? Retrieved Sept 12, 2018 from https://www . obesityaction.org/get-educated/understanding-childhood-obesity/ what-is-childhood-obesity/, 2019.

[11] WHO | Physical activity and young people. Retrieved March 25, 2019 from https://www. who.int/dietphysicalactivity/factsheet_young_ people/en/, 2019.

[12] Marc A. Adams, William D. Johnson, and Catrine Tudor-Locke. Steps/day translation of the moderate-to-vigorous physical activity guideline for children and adolescents. International Journal of Behavioral Nutrition and Physical Activity, 10(1):49, April 2013.

[13] Stephanie Alley, Stephanie Schoeppe, Diana Guertler, Cally Jennings, Mitch J Duncan, and Corneel Vandelanotte. Interest and preferences for using advanced physical activity tracking devices: results of a national cross-sectional survey. BMJ Open, 6(7), 2016. 
[14] Tim Althoff, Ryen W. White, and Eric Horvitz. Influence of Pokmon Go on Physical Activity: Study and Implications. Journal of Medical Internet Research, 18(12):e315, 2016.

[15] Ionut Andone, Konrad Blaszkiewicz, Matthias Bhmer, and Alexander Markowetz. Impact of location-based games on phone usage and movement: a case study on Pokmon GO. page 102. ACM, September 2017.

[16] Eva Böhler and Joachim Schüz. Cellular telephone use among primary school children in Germany. European Journal of Epidemiology, 19(11):1043-1050, November 2004.

[17] Amy Bruckman and Alisa Bandlow. The Human-computer Interaction Handbook. pages 428-440. L. Erlbaum Associates Inc., Hillsdale, NJ, USA, 2003.

[18] Patrick Burns, Christopher Lueg, and Shlomo Berkovsky. Activmon: Encouraging Physical Activity Through Ambient Social Awareness. In CHI '12 Extended Abstracts on Human Factors in Computing Systems, CHI EA '12, pages 2363-2368, New York, NY, USA, 2012. ACM. event-place: Austin, Texas, USA.

[19] James Clawson, Jessica A. Pater, Andrew D. Miller, Elizabeth D. Mynatt, and Lena Mamykina. No longer wearing: investigating the abandonment of personal health-tracking technologies on craigslist. In Proceedings of the 2015 ACM International Joint Conference on Pervasive and Ubiquitous Computing UbiComp '15, pages 647-658, Osaka, Japan, 2015. ACM Press.

[20] Jacqueline Howard CNN. 7 alternative games to Pokemon Go. Retrieved April 18, 2019 from https://www.cnn.com/2016/07/14/health/ pokemon-go-exercise-games-apps/index.html, 2019. 
[21] Sunny Consolvo, Predrag Klasnja, David W. McDonald, Daniel Avrahami, Jon Froehlich, Louis LeGrand, Ryan Libby, Keith Mosher, and James A. Landay. Flowers or a Robot Army?: Encouraging Awareness \& Activity with Personal, Mobile Displays. In Proceedings of the 10th International Conference on Ubiquitous Computing, UbiComp '08, pages 54-63, New York, NY, USA, 2008. ACM. event-place: Seoul, Korea.

[22] Sunny Consolvo, Ryan Libby, Ian Smith, James A. Landay, David W. McDonald, Tammy Toscos, Mike Y. Chen, Jon Froehlich, Beverly Harrison, Predrag Klasnja, Anthony LaMarca, and Louis LeGrand. Activity sensing in the wild: a field trial of ubifit garden. In Proceeding of the twenty-sixth annual CHI conference on Human factors in computing systems - CHI '08, page 1797, Florence, Italy, 2008. ACM Press.

[23] John W. Creswell. Qualitative inquiry and research design: choosing among five traditions. Sage Publications, 1998. Google-Books-ID: bjO2AAAAIAAJ.

[24] Allison Druin. Cooperative Inquiry: New Technologies for Children. page 8, 1999.

[25] Mitch Duncan, Beatrice Murawski, Camille E. Short, Amanda L. Rebar, Stephanie Schoeppe, Stephanie Alley, Corneel Vandelanotte, and Morwenna Kirwan. Activity Trackers Implement Different Behavior Change Techniques for Activity, Sleep, and Sedentary Behaviors. Interactive Journal of Medical Research, 6(2):e13, 2017.

[26] Robert E. Franken and Douglas J. Brown. Why do people like competition? The motivation for winning, putting forth effort, improving one's performance, 
performing well, being instrumental, and expressing forceful/aggressive behavior. Personality and Individual Differences, 19(2):175-184, August 1995.

[27] Michael I. Goran. Metabolic precursors and effects of obesity in children: a decade of progress, 19901999. The American Journal of Clinical Nutrition, 73(2):158-171, February 2001.

[28] Carlos Guestrin. Usability in machine learning at scale with graphlab. In Proceedings of the 22Nd ACM International Conference on Information $\&$ Knowledge Management, CIKM '13, pages 5-6, New York, NY, USA, 2013. ACM.

[29] Mona Leigh Guha, Allison Druin, Gene Chipman, Jerry Alan Fails, Sante Simms, and Allison Farber. Mixing ideas: A new technique for working with young children as design partners. In Proceedings of the 2004 Conference on Interaction Design and Children: Building a Community, IDC '04, pages 35-42, New York, NY, USA, 2004. ACM.

[30] Mona Leigh Guha, Allison Druin, and Jerry Alan Fails. Investigating the impact of design processes on children. In Proceedings of the 9th International Conference on Interaction Design and Children, IDC '10, pages 198-201, New York, NY, USA, 2010. ACM.

[31] Jeffrey Hartnett, Pearl Lin, Lillian Ortiz, and Lindsay Tabas. A responsive and persuasive audio device to stimulate exercise and fitness in children. In $C H I$ '06 extended abstracts on Human factors in computing systems - CHI EA '06, page 1837, Montr\&\#233;al, Qu\&\#233;bec, Canada, 2006. ACM Press.

[32] Koen Hendrix, Robert van Herk, Janneke Verhaegh, and Panos Markopoulos. Increasing Children's Social Competence Through Games, an Exploratory Study. 
In Proceedings of the 8th International Conference on Interaction Design and Children, IDC '09, pages 182-185, New York, NY, USA, 2009. ACM. event-place: Como, Italy.

[33] Mary C. Hooke, Laura Gilchrist, Lynn Tanner, Nicole Hart, and Janice S. Withycombe. Use of a Fitness Tracker to Promote Physical Activity in Children With Acute Lymphoblastic Leukemia. Pediatric Blood $\&$ Cancer, 63(4):684-689, April 2016.

[34] Ian Janssen. Physical activity guidelines for children and youth. Applied Physiology, Nutrition, and Metabolism, 32(S2E):S109-121, 2007.

[35] Ian Janssen. Healthy living and healthy weight. Healthy Settings for Young People in Canada. Retrieved from http://www. phac-aspc. gc. ca/hp-ps/dcadea/publications/yjc/pdf/youth-jeunes-eng. pdf, 2008.

[36] Ellie Jones, Lauren Seki, and Courtney Mostul. Prevalence and Use of Fitness Tracking Devices within a College Community. Linfield College Student Symposium: A Celebration of Scholarship and Creative Achievement, May 2017.

[37] Charlotte Kerner and Victoria A. Goodyear. The Motivational Impact of Wearable Healthy Lifestyle Technologies: A Self-determination Perspective on Fitbits With Adolescents. American Journal of Health Education, 48(5):287-297, September 2017.

[38] Christine Keung, Alexa Lee, Shirley Lu, and Megan O’Keefe. BunnyBolt: A Mobile Fitness App for Youth. In Proceedings of the 12th International Conference on Interaction Design and Children, IDC '13, pages 585-588, New York, NY, USA, 2013. ACM. event-place: New York, New York, USA. 
[39] Allana G LeBlanc, Peter T Katzmarzyk, Tiago V Barreira, Stephanie T Broyles, Jean-Philippe Chaput, Timothy S Church, Mikael Fogelholm, Deirdre M Harrington, Gang Hu, Rebecca Kuriyan, et al. Correlates of total sedentary time and screen time in 9-11 year-old children around the world: the international study of childhood obesity, lifestyle and the environment. PloS one, 10(6):e0129622, 2015.

[40] Dan Ledger and Daniel McCaffrey. Inside wearables: How the science of human behavior change offers the secret to long-term engagement. Endeavour Partners, 200(93):1, 2014.

[41] Victor R. Lee and Joel Drake. Quantified Recess: Design of an Activity for Elementary Students Involving Analyses of Their Own Movement Data. In Proceedings of the 12th International Conference on Interaction Design and Children, IDC '13, pages 273-276, New York, NY, USA, 2013. ACM.

[42] Victor R. Lee, Joel R. Drake, Ryan Cain, and Jeffrey Thayne. Opportunistic Uses of the Traditional School Day Through Student Examination of Fitbit Activity Tracker Data. In Proceedings of the 14 th International Conference on Interaction Design and Children, IDC '15, pages 209-218, New York, NY, USA, 2015. ACM.

[43] Chantal Lidynia, Philipp Brauner, and Martina Ziefle. A Step in the Right Direction- Understanding Privacy Concerns and Perceived Sensitivity of Fitness Trackers. In Tareq Ahram and Christianne Falcão, editors, Advances in Human Factors in Wearable Technologies and Game Design, Advances in Intelligent Systems and Computing, pages 42-53. Springer International Publishing, 2018. 
[44] James J. Lin, Lena Mamykina, Silvia Lindtner, Gregory Delajoux, and Henry B. Strub. Fish'N'Steps: Encouraging Physical Activity with an Interactive Computer Game. In Proceedings of the 8th International Conference on Ubiquitous Computing, UbiComp'06, pages 261-278, Berlin, Heidelberg, 2006. SpringerVerlag. event-place: Orange County, CA.

[45] Abbey Lunney, Nicole R. Cunningham, and Matthew S. Eastin. Wearable fitness technology: A structural investigation into acceptance and perceived fitness outcomes. Computers in Human Behavior, 65:114-120, December 2016.

[46] Arwen M. Marker and Amanda E. Staiano. Better Together: Outcomes of Cooperation Versus Competition in Social Exergaming. Games for Health Journal, 4(1):25-30, October 2014.

[47] Anna Lisa Martin-Niedecken. Plunder planet: An adaptive single- and multiplayer fitness game environment for children and young adolescents. In Extended Abstracts of the 2018 CHI Conference on Human Factors in Computing Systems, CHI EA '18, pages D201:1-D201:4, New York, NY, USA, 2018. ACM.

[48] Kathryn Mercer, Melissa Li, Lora Giangregorio, Catherine Burns, and Kelly Grindrod. Behavior Change Techniques Present in Wearable Activity Trackers: A Critical Analysis. JMIR mHealth and uHealth, 4(2), April 2016.

[49] Jochen Meyer, Jutta Fortmann, Merlin Wasmann, and Wilko Heuten. Making Lifelogging Usable: Design Guidelines for Activity Trackers. In Xiangjian He, Suhuai Luo, Dacheng Tao, Changsheng Xu, Jie Yang, and Muhammad Abul Hasan, editors, MultiMedia Modeling, Lecture Notes in Computer Science, pages 323-334. Springer International Publishing, 2015. 
[50] ODPHP. 2016 United States Report Card on Physical Activity for Children and Youth Released. Retrieved Sept 12, 2018 from https://health.gov/news/blog-bayw/2016/11/ 2016-united-states-report-card-on-physical-activity-for-children-and-youth-re November 2016.

[51] World Health Organization. Tenfold increase in childhood and adolescent obesity in four decades: new study by Imperial College London and WHO. Saudi Medical Journal, 38(11):1162-1163, November 2017.

[52] Joann Pan. 10 Fitness Apps That Boost Your Stamina, Speed and Strength. Retrieved April 18, 2019 from https://mashable.com/2012/07/26/ fitness-apps/, 2019.

[53] Bernd Ploderer, Yaman Mazyed S. Alsahfi, and Stewart G. Trost. Technologies to Engage Young Children in Physical Activity: An Online Study of Parenting Practices. In Proceedings of the 30th Australian Conference on Computer-Human Interaction, OzCHI '18, pages 326-331, New York, NY, USA, 2018. ACM.

[54] Janet C. Read, Panos Markopoulos, Narcis Pars, Juan Pablo Hourcade, and Alissa N. Antle. Child Computer Interaction. In CHI '08 Extended Abstracts on Human Factors in Computing Systems, CHI EA '08, pages 2419-2422, New York, NY, USA, 2008. ACM.

[55] Nicola D Ridgers, Melitta A McNarry, and Kelly A Mackintosh. Feasibility and Effectiveness of Using Wearable Activity Trackers in Youth: A Systematic Review. JMIR mHealth and uHealth, 4(4), November 2016. 
[56] Nicola D Ridgers, Anna Timperio, Helen Brown, Kylie Ball, Susie Macfarlane, Samuel K Lai, Kara Richards, Kelly A Mackintosh, Melitta A McNarry, Megan Foster, and Jo Salmon. Wearable Activity Tracker Use Among Australian Adolescents: Usability and Acceptability Study. JMIR mHealth and uHealth, 6(4), April 2018.

[57] Herman Saksono, Carmen Castaneda-Sceppa, Jessica Hoffman, Magy Seif ElNasr, Vivien Morris, and Andrea G. Parker. Family Health Promotion in Low-SES Neighborhoods: A Two-Month Study of Wearable Activity Tracking. In Proceedings of the 2018 CHI Conference on Human Factors in Computing Systems, CHI '18, pages 309:1-309:13, New York, NY, USA, 2018. ACM.

[58] Ankita Samariya and Jerry A. Fails. Making Physical Activity Fun \& Playful: Investigating Fitness Games with Children. In 20th International Conference on Human-Computer Interaction with Mobile Devices and Services, Barcelona,Spain, 2018. ACM.

[59] Roger Seiler and Marcel H'uttermann. E-HEALTH, FITNESS TRACKERS AND WEARABLES — USE AMONG SWISS STUDENTS. March 2015.

[60] Sarah Justine Guy Skriloff, Dario C. Gonzalez, Kurtis C. Christensen, Logan J. Bentley, and Cody V. Mortensen. FitPlay Games: Increasing Exercise Motivation Through Asynchronous Social Gaming. In Proceedings of the 2016 CHI Conference Extended Abstracts on Human Factors in Computing Systems, CHI EA '16, pages 164-167, New York, NY, USA, 2016. ACM.

[61] Catrine Tudor-Locke, Cora L Craig, Michael W Beets, Sarahjane Belton, Greet M Cardon, Scott Duncan, Yoshiro Hatano, David R Lubans, Timothy S 
Olds, Anders Raustorp, David A Rowe, John C Spence, Shigeho Tanaka, and Steven N Blair. How many steps/day are enough? for children and adolescents. The International Journal of Behavioral Nutrition and Physical Activity, 8:78, July 2011.

[62] Peter West, Max Van Kleek, Richard Giordano, Mark J. Weal, and Nigel Shadbolt. Common Barriers to the Use of Patient-Generated Data Across Clinical Settings. In Proceedings of the 2018 CHI Conference on Human Factors in Computing Systems, pages 484:1-484:13, New York, NY, USA, 2018. ACM.

[63] Bieke Zaman, Vero Vanden Abeele, and Dirk De Grooff. Measuring product liking in preschool children: An evaluation of the Smileyometer and This or That methods. International Journal of Child-Computer Interaction, 1(2):61-70, May 2013. 


\section{APPENDIX A}

\section{KIDFIT API'S}

KidFit API's facilitate the communication of data from the mobile applications to the database and the Fitbit server. This appendix details the list of the KidFit API's, the expected inputs and outputs and their basic functions.

\section{- direct_login.php}

This API uses the stored credentials in the app to present user with their fitness data and their games.

- Inputs - email address, access token

- Outputs - login status and fitness data of user

\section{- verify_login.php}

This API verifies the user credential when the user tries to login or encrypts and stores user credentials in the database when they register. Upon successful login or registration, login status and access token is returned.

- Inputs - email address, password, age , gender and alias

- Outputs - login status, access token

\section{- getInvitedGames.php}

For a given user, this API fetches all such games with a given game type (GTG 
or CTC) from the database where the user is invited or ready to play and the game status is invited.

- Inputs - email address, access token, gameID

- Outputs - number of invites games, gameInstanceID, groupName, string of player names, and self status for each game

\section{- getActiveGames.php}

For a given user, this API fetches all such games with a given game type (GTG or CTC) from the database where the user is ready to play or in progress and the game status is ready to play or in progress.

- Inputs - email address, access token, gameID

- Outputs - number of games with status ready to play or in progress, gameInstanceID, groupName, string of player names and status of each game.

\section{- getFinishedGames.php}

For a given user, this API fetches all such games with a given game type (GTG or CTC) from the database where the game status is game over.

- Inputs - email address, access token, gameID

- Outputs - number of games with status game over, gameInstanceID, groupName, string of player names and player status in each game

\section{- getCurrentActivity.php}

This API simply returns the number of steps and active minutes for a given user. 
- Inputs - email address, access token

- Outputs - steps, active minutes

- acceptGameInvite.php

This API lets a user accept a game invite that they have been invited to and returns success or error in response.

- Inputs - email address, access token, gameInstanceID

- Outputs - status

\section{- declineGameInvite.php}

This API lets a user accept a game invite that they have been invited to and returns success or error in response.

- Inputs - email address, access token, gameInstanceID

- Outputs - status

\section{- get_gameID.php}

This API takes the name of the game and activity metric and returns its ID which is then used to fetch all game details.

- Inputs - gameName, metric

- Outputs - gameID

\section{- resetPassword.php}

This API helps a user reset their password. The API takes email and gameName as input and returns an access code which is used to check against the code entered by user. 
- Inputs - email address, gameName

- Outputs - code

- updatePassword.php

This API facilitates updating user password and returns status as success or fail.

- Inputs - email address, new password

- Outputs - status

- getPlayersFromGroupID.php

This API returns the name of all players in a group or game.

- Inputs - email address, access token, groupID

- Outputs - number of players, name and userID of each player

- addPlayerToGameInstance.php

This API facilitates lets a user to add their friend to a game.

- Inputs - email address, access token, gameInstanceID, friendEmail

- Outputs - status

- removePlayerFromGameInstance.php

This API facilitates lets a user to remove their friend from a game.

- Inputs - email address, access token, gameInstanceID, friendID

- Outputs - status 


\section{- createGameInstance.php}

This API lets a user create a new game by inviting their friends. Only one friend can be invited while creating a game, more friends are added by using addPlayerToGameInstance.php. The new gameInstanceID is returned

- Inputs - email address, access token, gameID, friendEmail

- Outputs - status, gameInstanceID

\section{- editGroupName.php}

This API lets users in a group to edit the name of the group.

- Inputs - email address, access token, gameInstanceID, newGroupName

- Outputs - status

\section{- getGameDetails.php}

This API is used get the status of the game and all the players in the game.

- Inputs - email address, access token, gameInstanceID

- Outputs - groupName, number of players, gameOwner, username, userID and status of each player in the game.

\section{- getActiveGameDetail.php}

This API returns the details to an ongoing game.

- Inputs - email address, access token, gameInstanceID, gameID

- Outputs - timeRemaining, activeUserID, activeUserName, activity to achieve in a group game, cumulative active at the start of the game and now, endValue to be achieved, gameStatus and activity achieved by each player. 
- startGame.php

This API lets users in the game to start it such that the game status should be ready to play.

- Inputs - email address, access token, gameInstanceID, gameID

- Outputs - status, timeRemaining, activeUser, activeUserName, activity to win the game, currentActivity, endValue to be achieved

- gameOver.php

This API ends the game, when the allotted time is over or the group has won the game in collaborative type of play.

- Inputs - email address, access token, gameInstanceID

- Outputs - status, gameInstanceID, number of players, and user status. 


\section{APPENDIX B}

\section{MECHANICAL TURK SURVEY QUESTIONNAIRE}

This appendix lists the questions asked in children's fitness tracker survey conducted via Amazon's Mechanical Turk. The Survey was designed using Qualtrics and was link with MTurk. The survey starts with a consent to participate in the survey . If the parents consent, they first answer the generic questions (see Section B.1),followed by their children's fitness tracker usage questions (see Section B.2) for each of their child.

\section{B.1 Generic Questions Related to Parents}

- Parent's Age:

- Parent's Gender:

- Do you currently use a Fitness Tracker?

- How many children do you have between 6-11 yrs of age?

- Ages of children between 6-11 yrs of age (only provided options between 6 to 11 to choose from) 


\section{B.2 Questions Related to Children's Fitness tracker usage}

- Child's gender:

- Child's weight in pounds:

- Child's height in feet and inches:

- In the last 7 days, How many days did your child participate in any physical activity?

- In the last 7 days, What did your child do as part of their physical activity or outdoor active play (e.g. biking/walking to school, active outdoor play, sports, active video games)? How many days did they perform the activity?How much time (on average) did they spend per active day?

- Does your child use technology when he/she is active (e.g. a smartphone app, an active game like with Kinect or Wii)?

- What technology do they use?

- Has your child ever used a fitness tracker/pedometer? (The options were currently uses, Used in the past and Never used)

- If your child used fitness tracker in the past, How many months did they use it?

- If your child used a fitness tracker in the past but no longer use it, Why did they stop?

- If your child uses a fitness tracker currently, what is it (Choose all that apply)? (some popular options were given) 
- How do they use it?

- Who looks at the daily activity of the child (Choose all that apply)? (The options included Parents, child or others)

- What does you child use the data for (Choose all that apply)? (The option were Tracking goals, discussions, personal challenges, rewards, competition, don't use data and others)

- What do you (as a parent) or others use the data for (Choose all that apply)? (Same options as prev question)

- Who charges the device if it needs charging (Choose all that apply)?(The options were child, parent, the device doesn't need charging and others)

- If your child currently uses a tracker, how many months have they been using it?

- Why did you consider buying a fitness tracker?

- Choose the answer that best describes your perception as a parent: "My child is more active because of using a fitness tracker." (The options were strongly disagree, somewhat disagree, neither agree nor disagree, somewhat agree and strongly agree)

- Choose the answer that best describes your perception as a parent: "My child was more active because of using a fitness tracker." (Same options as previous question)

- Would you consider using a fitness tracker to help your child monitor their activity? 
- Please explain why you would/would not using a fitness tracker to help your child monitor their activity.

- How would you categorize the lifestyle of your child in terms of physical activity? (The options were sedentary, moderately active and highly active)

- How much screen time does your child get per day? Screen time is the time spent looking at computer screens, mobile phones, tablets or video games or other such displays.(The options were less than 30 minutes, 30-60 minutes, 60-90 minutes, 90-120 minutes and more than 120 minutes.)

- How might technology be used to encourage your children to be more active? 